Review

\title{
Simulating Groups and the IntraGroup Medium: The Surprisingly Complex and Rich Middle Ground between Clusters and Galaxies
}

\author{
Benjamin D. Oppenheimer ${ }^{1,2, * \mathbb{D}}$, Arif Babul ${ }^{3} \mathbb{D}$, Yannick Bahé ${ }^{4}$, Iryna S. Butsky ${ }^{5}$ and Ian an. McCarthy $^{6}(\mathbb{D}$ \\ 1 CASA, Department of Astrophysical and Planetary Sciences, University of Colorado, 389 UCB, \\ Boulder, CO 80309, USA \\ 2 Harvard Smithsonian Center for Astrophysics, 60 Garden Street, Cambridge, MA 02138, USA \\ 3 Department of Physics and Astronomy, University of Victoria, Victoria, BC V8W 2Y2, Canada; babul@uvic.ca \\ 4 Leiden Observatory, Leiden University, P.O. Box 9513, 2300 RA Leiden, The Netherlands; \\ bahe@strw.leidenuniv.nl \\ 5 Astronomy Department, University of Washington, Seattle, WA 98195, USA; ibutsky@uw.edu \\ 6 Astrophysics Research Institute, Liverpool John Moores University, 146 Brownlow Hill, Liverpool L53RF, UK; \\ i.g.mccarthy@ljmu.ac.uk \\ * Correspondence: benjamin.oppenheimer@colorado.edu
}

\section{check for} updates

Citation: Oppenheimer, B.D.; Babul, A.; Bahé, Y.; Butsky, I.S.; McCarthy, I.G. Simulating Groups and the IntraGroup Medium: The Surprisingly Complex and Rich Middle Ground between Clusters and Galaxies. Universe 2021, 7, 209. https://doi.org/10.3390/ universe7070209

Academic Editors: Lorenzo Lovisari and Stefano Ettori

Received: 9 April 2021

Accepted: 2 June 2021

Published: 24 June 2021

Publisher's Note: MDPI stays neutral with regard to jurisdictional claims in published maps and institutional affiliations.

Copyright: (c) 2021 by the authors. Licensee MDPI, Basel, Switzerland. This article is an open access article distributed under the terms and conditions of the Creative Commons Attribution (CC BY) license (https:// creativecommons.org/licenses/by/ $4.0 /)$.
Abstract: Galaxy groups are more than an intermediate scale between clusters and halos hosting individual galaxies, they are crucial laboratories capable of testing a range of astrophysics from how galaxies form and evolve to large scale structure (LSS) statistics for cosmology. Cosmological hydrodynamic simulations of groups on various scales offer an unparalleled testing ground for astrophysical theories. Widely used cosmological simulations with $\sim(100 \mathrm{Mpc})^{3}$ volumes contain statistical samples of groups that provide important tests of galaxy evolution influenced by environmental processes. Larger volumes capable of reproducing LSS while following the redistribution of baryons by cooling and feedback are the essential tools necessary to constrain cosmological parameters. Higher resolution simulations can currently model satellite interactions, the processing of cool ( $T \approx 10^{4-5} \mathrm{~K}$ ) multi-phase gas, and non-thermal physics including turbulence, magnetic fields and cosmic ray transport. We review simulation results regarding the gas and stellar contents of groups, cooling flows and the relation to the central galaxy, the formation and processing of multi-phase gas, satellite interactions with the intragroup medium, and the impact of groups for cosmological parameter estimation. Cosmological simulations provide evolutionarily consistent predictions of these observationally difficult-to-define objects, and have untapped potential to accurately model their gaseous, stellar and dark matter distributions.

Keywords: black holes; galaxy groups; galaxy surveys; intragroup medium/plasma; hydrodynamical and cosmological simulations; active galactic nuclei; X-ray observations; UV observations; cosmological parameters

\section{Introduction}

Galaxy groups are versatile laboratories to study a range of astrophysics spanning nongravitational, baryonic processes associated with galaxy formation to large-scale structure statistics constraining cosmology. Their intermediate scale between galactic halos and clusters offers a unique set of theoretical challenges that are often overlooked relative to adjacent mass bins, but this scale offers crucial constraints for how a large proportion of galaxies evolved to their present state. The perspective of this review, focusing on halos with masses $M_{\text {halo }} \approx 10^{13}-10^{14} \mathrm{M}_{\odot}$, differs from the companion reviews because a cosmological simulation tracks the evolution of all gas, stars and dark matter, not just the X-ray emitting intragroup medium (IGrM). Therefore we consider all phases of gas-from the extended, hot IGrM that stretches beyond the virial radius to the cold interstellar 
medium (ISM) within individual group galaxies-alongside the stars and dark matter in central and satellite group galaxies, as well as the surrounding cosmological large scale structure (LSS). Simulations have enabled breakthroughs in our understanding of all these components, but their limited resolution and incomplete physics models still represent a major obstacle on the path to a truly complete understanding of the observed gas, galaxies and LSS statistics in and around groups.

The IGrM is mainly very hot $\left(T \sim 10^{7} \mathrm{~K}\right)$, but does not primarily radiate via Bremsstrahlung radiation like the intracluster medium (ICM; see Figure 1 of the companion review by Lovisari et al. [1]). Cooling via line emission offers a greater opportunity to form multi-phase, cool ( $\left.T<10^{5} \mathrm{~K}\right)$ gas, and potentially provide additional fuel to galaxies. Nevertheless, IGrM observations are currently dominated by hot X-ray probes from especially Chandra [2-4] and XMM-Newton (e.g., Lovisari et al. [5]). X-ray-derived profiles of IGrM properties, especially its entropy, provide rigorous tests for simulations that often make diverging predictions (e.g., Mitchell et al. [6], Le Brun et al. [7]). Yet these simulations also produce lower mass groups that can be tested against the Complete Local Volume Groups Sample (CLoGS) survey [8], and sometimes higher mass objects for comparison with observed clusters (e.g., $[9,10])$. With large-scale simulations projects containing multiple volumes and/or zoom-in simulations of massive objects, simulations of the IGrM can be compared and contrasted to lower mass galaxy halos and higher mass clusters.

Similar to a cluster, most groups contain a dominant "brightest group galaxy" (BGG) near the halo center, usually where the X-ray-traced IGrM peaks in brightness. In contrast to brightest cluster galaxies (BCGs), BGGs are observed to less likely be quenched early-type galaxies (ETGs; e.g., [11,12]), and more likely to have disc-like morphologies (e.g., [13]). Understanding the-likely intimate-connection between the BGG and IGrM involves studying how the galactic baryon cycle (the interplay of gas accretion, outflows, and recycling that is understood as fundamental for the co-evolution of field galaxies and their circumgalactic medium; $[14,15])$ transitions to the cluster version of precipitation, jet-driven active galactic nuclei (AGN) feedback, and chaotic cold accretion (e.g., [16,17]).

The IGrM also includes cool and warm $\left(T \sim 10^{5}-10^{6} \mathrm{~K}\right)$ gas phases. Although subdominant to the hot IGrM component by mass as simulations clearly demonstrate (e.g., [18,19]), these phases are thought to represent the link between the IGrM and individual group galaxies: from them, gas can accrete onto the BGG and fuel further star formation (e.g., [20,21]), whereas gas stripped from satellite galaxies (e.g., [22-24]) or ejected from them through superwind feedback (e.g., [25]) is also initially less hot than the virialized IGrM halo. Furthermore, observations of quasar absorption lines in the UV indicate a substantial reservoir of $\mathrm{HI}$ and metals in the IGrM at temperatures of $10^{4}-10^{5.5} \mathrm{~K}[26,27]$, and 21-cm emission shows extended IGrM structures, at least for more compact, spiral-rich groups [28]. The theoretical modeling of cool/warm gas is crucial to understand for how groups diverge from clusters owing to their lower temperatures promoting more cooling.

Lower IGrM pressures and galaxy velocities process satellite galaxies differently than in clusters: simulations find both the atomic hydrogen and star forming gas is removed less rapidly after infall [29], providing an explanation for observed group galaxies being less $\mathrm{H} \mathrm{I}$ deficient than in clusters [30] and having lower quenched fractions (e.g., [11,12]). However, the lower velocity dispersion of group satellites makes dynamical friction more efficient, so that mergers-in particular between satellites and the BGG-are more common [31]. Finally, although groups are (typically) dynamically older than clusters, their galaxies are more likely to have been accreted directly from the field, rather than via an intermediate "pre-processing" phase in a lower-mass halo (e.g., [32]). The total time that $z=0$ galaxies have spent as satellites is therefore $\approx 50 \%$ shorter for groups than massive clusters (ca. 4 vs. 6 Gyr; [33], see also Donnari et al. [34]).

Relative to clusters, the $\sim 10 \times$ higher number density of groups (e.g., [35]) combined with their shallower potential wells - which make it easier for AGN feedback to eject baryons-gives groups particular significance for cosmological parameter estimates that rely on the total mass distribution, for example, lensing measurements, cosmic shear, 
and redshift space distortions. The distribution of baryons in halos corresponding to groups $\left(10^{13-14} \mathrm{M}_{\odot}\right)$ is known to be significantly affected by feedback processes. Therefore, the selfconsistent modeling of the entire baryonic (gas+stellar) and dark matter distributions of groups, in way that realistically captures the effects of galaxy formation, is a necessary tool for precision cosmology.

In this review, we focus on how groups process their baryons in a cosmological context, and therefore discuss predominantly cosmological simulations that contain groups. Entire separate reviews could be written about idealized simulations and analytical models at the group scale, but these are not able to confront the intersection of galaxy formation and cosmology. We will, however, refer to idealized simulations and other methods simulating important physics when they are relevant to groups, especially in regards to how cosmological simulations can improve. Ultimately, our understanding of how gas and galaxies evolve together to create the observed distribution of groups would not be as far along without these tools.

Throughout this review, we use a theorist's definition of a group as a system of galaxies and gas hosted by a halo within a certain mass range. Following common convention, we define these masses as $M_{\Delta}$, the sum of all matter species (i.e., dark matter and baryons) within a spherical aperture $r_{\Delta}$ inside which the mean density equals $\Delta$ times the critical density of the universe; we adopt the value $\Delta=500$ as commonly used in X-ray studies of the IGrM/ICM and thus define a group halo as one with $M_{500(\mathrm{c})}=10^{13}-10^{14} \mathrm{M}_{\odot}$ $\left(r_{500(\mathrm{c})} \approx 340-600 \mathrm{kpc}\right)^{1}$. This definition places groups between lower-mass galactic halos $\left(M_{500} \lesssim 10^{13} \mathrm{M}_{\odot}\right)$ and more massive clusters $\left(M_{500} \gtrsim 10^{14} \mathrm{M}_{\odot}\right)$. For the benefit of readers more used to masses within other overdensity thresholds, we note that in this halo mass range masses calculated within spheres of average density equal to 200 times the critical, or 200 times the mean, density $\left(M_{200}\right.$ and $M_{200 m}$, respectively), or to the virial overdensity $\Delta_{\mathrm{vir}} \approx 18 \pi^{2}+82(\Omega(z)-1)-39(\Omega(z)-1)^{2}$ [36] that is based on the analytic solution of spherical top-hat collapse $\left(M_{\mathrm{vir}}\right)$, are offset from $M_{500}$ by $+0.16 \operatorname{dex}\left(M_{200}\right),+0.31$ $\operatorname{dex}\left(M_{200 m}\right)$, and +0.25 dex $\left(M_{\mathrm{vir}}\right)$, respectively (and the corresponding radii from $r_{500}$ by factors of 1.5, 2.6, and 2.1 $)^{2}$. A typical group with mass of $M_{500}=10^{13.5} \mathrm{M}_{\odot}$ has a radius $R_{500}=480 \mathrm{kpc}$, a virial temperature $T_{\mathrm{X}} \sim 1 \mathrm{keV}$, and a velocity dispersion $\sigma \approx 440 \mathrm{~km} \mathrm{~s}^{-1}$ at $z=0$.

This review is organized into sections as follows: We begin with an overview of the cosmological simulations we discuss in Section 2. The methods used in simulation of groups are discussed in a series of subsections throughout Section 3. Section 4 comprises the results of current simulations creating groups, and is divided into five main subsections: the baryonic content of groups (Section 4.1), the connection between the central galaxy and the IGrM (Section 4.2), the multiphase IGrM (Section 4.3), satellite galaxies in groups (Section 4.4), and the impact of galaxy group astrophysics on LSS cosmology (Section 4.5). We discuss future directions in Section 5 and make a short final statement in Section 6.

\section{Overview of Simulations That Model Groups}

Cosmological simulations use a variety of hydrodynamics schemes, span many orders of magnitude in mass and spatial resolution, and model volumes of vastly different sizes and contents. This rich diversity in modeling approaches reflects the wide range of astrophysical processes and objects that different simulations attempt to model. In Table 1, we list recent simulations that include galaxy groups and are therefore of particular interest to our review. 
Table 1. Modern cosmological hydrodynamic simulations with groups that run to $z=0$.

\begin{tabular}{|c|c|c|c|c|c|c|c|}
\hline Simulation & $\begin{array}{l}\text { Simulation } \\
\text { Code }\end{array}$ & $\begin{array}{l}\text { Hydrodynamic } \\
\text { Scheme }\end{array}$ & $\begin{array}{l}\text { Baryon } \\
\text { Resolution } \\
\left(\mathbf{M}_{\odot}\right)\end{array}$ & $\begin{array}{l}\text { Volume } \\
\left(\mathrm{Mpc}^{3}\right)\end{array}$ & $\begin{array}{l}\text { AGN } \\
\text { Feedback } \\
\text { Scheme }\end{array}$ & $\begin{array}{l}f_{\text {gas,500 }} \text { at } \\
M_{500}= \\
10^{13.5} \mathrm{M}_{\odot}\end{array}$ & $\begin{array}{l}f_{*, 500} \text { at } \\
M_{500}= \\
10^{13.5} \mathbf{M}_{\odot}\end{array}$ \\
\hline cosmo-OWLS a & GADGET-3 & Classical SPH & $1.2 \times 10^{9}$ & $2.1 \times 10^{8}$ & Thermal & 0.05 & \\
\hline Illustris ${ }^{b}$ & AREPO & Moving Mesh & $1.3 \times 10^{6}$ & $1.2 \times 10^{6}$ & Dual & 0.01 & 0.04 \\
\hline EAGLE $^{c}$ & GADGET-3 & Modern SPH & $1.8 \times 10^{6}$ & $1.0 \times 10^{6}$ & Thermal & 0.11 & 0.01 \\
\hline Liang et al. [37] & GADGET-2 & Classical SPH & $9.0 \times 10^{7}$ & $2.9 \times 10^{6}$ & None & 0.09 & 0.06 \\
\hline Horizon-AGN ${ }^{\mathrm{d}}$ & RAMSES & AMR & $1 \times 10^{7}$ & $2.9 \times 10^{6}$ & Dual & 0.09 & \\
\hline BAHAMAS ${ }^{\mathrm{e}}$ & GADGET-3 & Classical SPH & $1.2 \times 10^{9}$ & $2.1 \times 10^{8}$ & Thermal & 0.04 & 0.02 \\
\hline C-EAGLE/Hydrangea ${ }^{f}$ & GADGET-3 & Modern SPH & $1.8 \times 10^{6}$ & 30 zooms & Thermal & 0.08 & 0.02 \\
\hline FABLE $g$ & AREPO & Moving Mesh & $9.4 \times 10^{6}$ & $2.0 \times 10^{5}+6$ zooms & Dual & 0.07 & 0.02 \\
\hline The Three Hundred ${ }^{\mathrm{h}}$ & GADGET-3 & Modern SPH & $3.5 \times 10^{8}$ & 324 zooms & Dual & 0.10 & 0.02 \\
\hline IllustrisTNG100 ${ }^{\mathrm{i}}$ & AREPO & Moving Mesh & $1.4 \times 10^{6}$ & $1.4 \times 10^{6}$ & Dual & 0.08 & 0.02 \\
\hline IllustrisTNG300 j & AREPO & Moving Mesh & $1.1 \times 10^{7}$ & $2.8 \times 10^{7}$ & Dual & 0.08 & \\
\hline ROMULUS k & CHANGA & Modern SPH & $2.1 \times 10^{5}$ & $1.5 \times 10^{4}+3$ zooms & Thermal & 0.11 & 0.04 \\
\hline SIMBA $^{1}$ & GIZMO & Meshless Finite Mass & $1.8 \times 10^{7}$ & $2.9 \times 10^{6}$ & Dual & 0.04 & 0.02 \\
\hline IllustrisTNG50 m & AREPO & Moving Mesh & $8.5 \times 10^{4}$ & $1.4 \times 10^{5}$ & Dual & 0.09 & \\
\hline Magneticum-Box $2 / \mathrm{hr}^{\mathrm{n}}$ & GADGET-3 & Modern SPH & $1.4 \times 10^{8}$ & $1.3 \times 10^{8}$ & Dual & & \\
\hline
\end{tabular}

${ }^{a}$ Le Brun et al. [7]; ${ }^{b}$ Vogelsberger et al. [38]; ${ }^{c}$ Schaye et al. [39]; ${ }^{d}$ Dubois et al. [40]; ${ }^{\mathrm{e}}$ McCarthy et al. [41]; ${ }^{\mathrm{f}}$ Bahé et al. [42], Barnes et al. [43]; ${ }^{\mathrm{g}}$ Henden et al. [44]; ${ }^{\mathrm{h}}$ Cui et al. [45]; ${ }^{\mathrm{i}}$ Pillepich et al. [46]; ${ }^{j}$ Nelson et al. [47]; ${ }^{\mathrm{k}}$ Tremmel et al. [48,49]; ${ }^{1}$ Davé et al. [50]; ${ }^{\mathrm{m}}$ Nelson et al. [51]; ${ }^{\mathrm{n}}$ http:/ / www.magneticum.org (accessed on 10 June 2021).

This list encompasses simulations with three broad classes of hydrodynamics schemes, as indicated in the third column of Table 1. Adaptive Mesh Refinement (AMR; [52]) uses a fixed (Eulerian) grid whose cells are locally and dynamically (de-/)refined, typically depending on the local gas density. Smoothed Particle Hydrodynamics (SPH; [53,54]), on the other hand, is a Lagrangian scheme in which gas mass is discretized into a finite number of particles that move under the influence of gravity and hydrodynamic forces; the latter are calculated by smoothing over neighboring particles. The third class is a hybrid of these two: in the "moving mesh" approach [55], an unstructured grid is used that moves with the local gas flow, whereas in "meshless finitie mass" simulations ([56], see also [57]), mass is discretized into particles like in SPH, but with explicit accounting of mass flows between neighboring particles ${ }^{3}$. We note that, as we discuss in Section 3.1, early SPH implementations suffered from systematic problems that have motivated improved "modern" formulations of SPH (see e.g., [58-61]); most SPH simulations that we discuss use one of these modern variants.

Many simulations listed in Table 1 evolve periodic, cosmological cubes with side length $\approx 100 \mathrm{Mpc}$, large enough to contain at least several dozen groups. In addition to modeling hydrodynamics and gravity, they all contain "subgrid" prescriptions for astrophysical processes that originate on unresolved scales, such as gas cooling, the formation of stars and black holes, and feedback associated with it (see Section 3). The "galaxy formation model" formed by these prescriptions is nowadays often calibrated to reproduce a particular set of galaxy properties. The Illustris [38], EAGLE (Evolution and Assembly of GaLaxies and their Environments; [39] ${ }^{4}$, and Horizon-AGN [40] simulations are representative examples of this approach. Their ability to (broadly) reproduce galactic stellar mass functions, galaxy colors and star formation rates (SFRs), and even galaxy morphologies signified a transformational advance over previous generations of simulations. Particularly relevant for groups, almost all the simulations listed in Table 1 model the accretion of gas onto SMBHs, and the resulting AGN feedback. In some recent simulations, including the IllustrisTNG project $[46,47]^{5}$ and SIMBA [50], these models are explicitly calibrated against gaseous properties of group-scale halos.

Simulations using the same or slightly modified codes as in some of the abovementioned projects target volumes $\gg 10^{6} \mathrm{Mpc}^{3}$, with the aim to model clusters and/or LSS. For example, the C-EAGLE/Hydrangea simulations [42,43] extend EAGLE with 30 "zoom-in" simulations centered on clusters with $M_{200}=10^{14.0}-10^{15.4} \mathrm{M}_{\odot}, 24$ of which (the Hydrangea suite) with high-resolution regions that extend to $10 r_{200}$ at $z=0$ [42]. The zoom technique allows these simulations to reach the same resolution $\left(\approx 2 \times 10^{6} \mathrm{M}_{\odot}\right.$ for baryons) as the 100 Mpc EAGLE "Reference" run, but with slightly adjusted AGN 
feedback parameters for more realistic gas fractions in groups as we detail in Section 3.8. The only simulation that reaches a comparable resolution in a full $\gg 10^{6} \mathrm{Mpc}^{3}$ volume is the TNG300 run of the IllustrisTNG suite: it evolves a $(\approx 300 \mathrm{Mpc})^{3}$ volume with a baryon mass resolution of $\approx 1.1 \times 10^{7} \mathrm{M}_{\odot}$, that is, $\approx 6$ or 8 times lower resolution than (C-)EAGLE or the $\approx 100 \mathrm{Mpc}$ TNG100 simulation, respectively. We note that, irrespective of this resolution difference, all TNG simulations use the same subgrid model and parameters. The degree of numerical convergence between these different resolution levels is discussed in for example, Pillepich et al. [67] and Donnari et al. [68]; we refer to Schaye et al. [39] for the opposite "weak convergence" approach of re-calibrating parameters for different resolution levels.

The cosmo-OWLS [7] and BAHAMAS (BAryons and HAloes of MAssive Systems) [41] simulations run larger periodic volumes at lower resolution. cosmo-OWLS varied different aspects of the subgrid models, switching different physics models on and off as well as changing the efficiencies of stellar and AGN feedback. No attempt was however made to calibrate the simulations to match observations, even though the default model reproduces different aspects of groups relatively well. BAHAMAS, on the other hand, explicitly calibrated the stellar and AGN feedback to reproduce the gas fractions of galaxy groups and the galaxy stellar mass function in order to ensure a realistic treatment of the effects of baryons on the matter power spectrum. The Magneticum simulations [69] are a series of volumes that mainly concentrate on LSS and cluster astrophysics. We list their Box $2 / \mathrm{hr}$ simulation in Table 1, described in Castro et al. [70], which is capable of resolving a statistical sample of groups in a volume $500 \mathrm{Mpc}$ on a side ${ }^{6}$. Lower resolution, Gpc-scale Magneticum volumes employ a strategy of calibrating subgrid modules to a Planck cosmology, and then exclusively varying cosmological parameters to explore the impact on baryonic properties. The Three Hundred project [45] simulated 324 clusters with $M_{\mathrm{vir}}(z=0) \gtrsim 1.2 \times 10^{15} \mathrm{M}_{\odot}$ out to a radius of $15 \mathrm{~h}^{-1} \mathrm{Mpc}$; similar to Hydrangea, the simulations therefore also contain many groups in the periphery of the central clusters.

We also list the Liang et al. [37] simulations and the Henden et al. [44] FABLE (Feedback Acting on Baryons in Large-scale Environments) simulations, both of which were run at lower resolution than their contemporary counterparts but with the aim to reproduce properties of groups and clusters. The former is an example of a simulation suite that does not include AGN feedback. In contrast, FABLE calibrated their subgrid models to reproduce the galactic stellar mass functions and, specifically, the gas and stellar contents of $M_{500} \approx 10^{13-15} \mathrm{M}_{\odot}$ halos. They simulated one smaller volume, supplemented by 6 zoom simulations extending up to a $M_{500}=10^{15} \mathrm{M}_{\odot}$ cluster.

Finally, we will also discuss two higher-resolution simulations that include groups: the ROMULUS suite $[48,49,72,73]$ and the IllustrisTNG50 (TNG50; [51,74]) simulation. ROMULUS includes a small-volume run, ROMULUS25, and three group/cluster zooms called ROMULUSG1, ROMULUSG2 and ROMULUSC, all at a baryon mass resolution of $2.1 \times 10^{5} \mathrm{M}_{\odot}$. TNG50 is a larger volume, $\approx 50 \mathrm{Mpc}$ on a side, which contains 20 halos with $M_{500}>10^{13} \mathrm{M}_{\odot}$ and reaches an even higher mass resolution of $8.5 \times 10^{4} \mathrm{M}_{\odot}$ for baryons.

Many other simulations have modeled groups from cosmological initial conditions, often with the zoom-in approach. These include the three $10^{13} \mathrm{M}_{\odot}$ group zooms by Feldmann et al. [75], which focus on the evolution and morphology of the central group galaxy; the 10 EAGLE-CGM zooms of $\sim 10^{13} \mathrm{M}_{\odot}$ groups by Oppenheimer et al. [76] to understand how the circumgalactic medium (CGM) around passive galaxies differs from star-forming galaxies at $10 \times$ lower halo mass, and the Joshi et al. [77] 2-3 $\times 10^{13} \mathrm{M}_{\odot}$ zoom to study environmental processing of satellite galaxies.

These simulations span a factor of 10,000 in both mass resolution and volume, and were run to study objects on a wide range of scales-from $M_{\star}<10^{8} \mathrm{M}_{\odot}$ dwarf galaxies to the $\gg$ Mpc scale LSS. However, they all overlap at the mass scale of our fiducial intermediatemass group with $M_{500}=10^{13.5} \mathrm{M}_{\odot}$. In Table 1, we therefore list the approximate total gas and stellar mass fractions inside $R_{500}$, defined as

$$
f_{\text {gas }, 500} \equiv \frac{M_{\text {gas }}\left(<R_{500}\right)}{M_{\text {tot }}\left(<R_{500}\right)}
$$


and

$$
f_{\star, 500} \equiv \frac{M_{\star}\left(<R_{500}\right)}{M_{\text {tot }}\left(<R_{500}\right) .}
$$

For a more detailed discussion of these fractions, and their dependence on halo mass, we refer the interested reader to Figures 14 and 15 in the companion review by Eckert et al. [78].

\section{Computational Methods Relevant for Simulations of Groups}

The cosmological simulations we discuss are N-body+hydro simulations beginning from cosmological initial conditions at $z \gtrsim 100$. They all contain cooling, star formation, and stellar feedback, which are necessary to form realistic galaxies. All but the Liang et al. [37] simulation include the SMBH seeding, SMBH accretion, and AGN feedback, which are necessary to reproduce key properties of groups. All Magneticum simulations include a passive magnetic field [79] and IllustrisTNG uses a magnetic hydrodynamic (MHD) solver that self-consistently follows the magnetic field, which is initially seeded in the initial conditions [80]. Separate code modules are written for these different aspects and while our discussion here is not an exhaustive list of code modules in every simulation, we focus on specific methods, prescriptions and subgrid models that impact the group scale, while providing some additional context.

\subsection{Hydrodynamics}

Historically, the choice of hydrodynamic scheme has had significant effects on the appearance of simulated clusters, in particular the entropy profiles near cluster centers. Grid-based, Eulerian mesh codes tend to produce higher entropy, flatter cores than particlebased schemes as first shown by Frenk et al. [81]. This behavior is believed to be a result of numerical diffusion and over-mixing in mesh codes and/or the absence of heat diffusion in "classical" SPH simulations [82]. Mitchell et al. [6] explored these phenomena using idealized cluster merger simulations in both FLASH (grid-based) and GADGET-2 (SPH), finding that the former creates flatter high-entropy cores by mixing entropy through vortices and turbulent eddies. They argued that the suppression of mixing and fluid instabilities (e.g., Kelvin-Helmholtz) in SPH simulations appear to preserve the entropy low of individual gas particles resulting in a power-law entropy distribution reminiscent of a cool core cluster.

Recent numerical improvements in modern SPH and AMR codes have greatly improved their ability to recreate a wider range of entropy profiles. For example, adding artificial conduction to GADGET-3 simulations allowed for the creation of cored profiles in SPH simulations by mimicking thermal diffusion, resulting in the production of both cool core (CC) and non-cool core (NCC) entropy profiles across a sample of massive cluster simulations [83]. Hahn et al. [84] ran a set of RAMSES AMR simulations which also produced the dichotomy of CC/NCC clusters. The nIFTy simulation code comparison project compared one $10^{15} \mathrm{M}_{\odot}$ cluster [85], finding that modern SPH methods could create entropy cores just as grid-based and moving mesh methods do. These updated SPH implementations, such as ANARCHY [59] used by EAGLE and the Beck et al. [60] scheme used by Magneticum, often include a combination of pressure-entropy formulations of $\mathrm{SPH}$, higher-order SPH kernels, and new treatments for thermal conduction and artificial viscosity. Cluster simulation comparison projects using modern SPH schemes (like the nIFTy project and The Three Hundred project [45]) now find that hydrodynamic scheme makes less of a difference than the inclusion of subgrid models on the appearance of entropy cores. While the appearance of entropy cores does not necessarily depend on the AGN feedback scheme at the cluster scale, the lower potentials of galaxy groups may yield a different answer.

The majority of the simulations explored here use either AREPO moving mesh or GADGET-2/GADGET-3 SPH, either classical or modern as listed in Table 1. The CHANGA code has a number of SPH updates putting it in the modern category. 


\subsection{Gas Cooling and Heating}

All simulations we discuss include radiative cooling and photoionization heating by the meta-galactic UV/X-ray background (UVB, for e.g., [86]). A module accesses cooling/photo-heating rate tables usually as a function of density, temperature and redshift. These lookup tables are usually calculated from CLOUDY [87] models element-by-element, where the redshift dependence accounts for the evolving UVB. Most of the GADGET3 simulations use Wiersma et al. [88] rates calculated for 11 elements, SIMBA uses the GRACKLE library [89] that tabulates self-shielding from the UVB for dense gas, and the AREPO-based simulations track additional photo-heating from local AGN that suppresses cooling in nearby gas [90].

For IGrM temperatures and densities that emit in the X-ray, cooling is driven by the balance of recombination and collisional ionization, with photo-ionization/heating usually playing an insignificant role. Often ionization equilibrium is assumed, but some simulations follow non-equilibrium rates of primordial elements (e.g., SIMBA). Cen and Fang [91] integrated non-equilibrium tracking of high oxygen ions ( $\mathrm{OV}-\mathrm{O}$ IX) in uniform mesh simulations, following how these ion species could deviate from equilibrium conditions when the recombination time became significant compared to the cooling time. Oppenheimer et al. [76] ran zoom SPH simulations following all ions from 11 metal species and including collisional [92], photo-ionization [93], Auger ionization and charge exchange [94] processes in low-mass groups. While non-equilibrium processes involving metals are not likely to significantly alter dynamics of cooling at IGrM temperatures, they can have an impact on the observational diagnostics, such as O VII and O VIII line emission [92,94]. Further exploration of IGrM simulations integrating non-equilibrium ionization and cooling is necessary in light of the coming launch of XRISM that will allow the measurement of line emission in the IGrM and ICM.

In contrast to simulations with radiative cooling, non-radiative (or adiabatic) simulations do not contain gas cooling (or any galaxy or SMBH formation). Frenk et al. [81], Lewis et al. [95], and Voit et al. [96] ran such simulations, finding that a baseline $K \propto R^{1.1}$ entropy profile is created in the absence of cooling, feedback, and other processes related to galaxy formation. ${ }^{7}$.

We also note that the ROMULUS simulations do not include metal-line cooling at $T>10^{4} \mathrm{~K}[48]$.

\subsection{Star Formation, Stellar Evolution, and Nucleosynthetic Production}

The formation of stars, their evolution and the release of elements are included in subgrid models for all the simulations we discuss. The multi-phase ISM is never resolved in separate phases, but relies on applying subgrid models that encompass multiple phases in a single gas parcel. A density threshold, above which the multi-phase ISM forms, is often the only criterion used for determining if gas can form stars. Different subgrid ISM models (e.g., [99-101]) use different motivations for their choices of unresolved phases, but all are calibrated to reproduce the Kennicutt [102] SFR surface density as a function of gas surface density relation. Star particles are spawned from gas parcels probabilistically and represent a population of individual equal-age stars, since they are at minimum $\sim 10^{5} \mathrm{M}_{\odot}$. Stellar death is modeled as a time-dependent return of a star particle's mass to surrounding gas as a function of star particle age, based on expectations from stellar evolution. The initial mass function (IMF) determines the proportion of stars that die and can induce a systematic shift in the stellar mass formed per amount of stellar luminosity emitted. Fortunately, the simulations used in the comparisons of $M_{*}$ in Section 4.2 use either a Kroupa [103] IMF (ROMULUS) or a Chabrier [104] IMF (every other simulation), which are fairly similar in their shapes.

The release of elements from stars is the source for the enrichment of the IGrM, about which an entire review by Gastaldello et al.[105] is written in this Special Issue "The Physical Properties of the Groups of Galaxies". In their Section 3.2, they include a discussion of chemical evolution models in cosmological hydrodynamic simulations. We note here that the simulations we discuss frequently all use chemical evolution models that include the 
elemental yields taken from stellar evolution models of Type II Supernovae (SNe), Type Ia $\mathrm{SNe}$, and AGB stars. We note that yields have a great deal of uncertainty associated with them as explained in Wiersma et al. [106]. Uncertainties arise in the production of iron from Type Ia SNe since the Type Ia rate is not well constrained, in the transition stellar mass from Type II SNe to AGB stars, in the shape of the IMF, and in the calculations of the elemental yields themselves. These uncertainties can directly affect the rates of gas cooling (Section 3.2), which can be dominated by metal-line emission at many IGrM temperatures (i.e., between $T \approx 10^{4.5}-10^{6.5} \mathrm{~K}$ for a $\mathrm{Z}=0.3 \mathrm{Z} \odot$ plasma, [92,94]).

\subsection{Metal Spreading}

The distribution of metals in the IGrM depends on (1) how metals are released from star particles into the surrounding gas; and (2) how metals diffuse between gas elements. This is a separate issue from stellar feedback that applies mechanical feedback energy to gas elements, which we discuss in the next section.

Metal enrichment to large distances can occur without mechanical feedback if the number of enrichment neighbors extends over a large volume, which may unrealistically enrich diffuse environments. In cases where the number of neighbors is large and/or the resolution is low, simulated star particles may artificially enrich diffuse environments like the IGrM without superwinds. This effect is most obvious in the uniform mesh simulations of Cen and Fang [91], where O VII and O VIII absorption statistics of diffuse gas remain at similar levels with and without superwinds. Modern simulations typically release metals over several dozen neighboring gas elements, based on either the smoothing kernel in the case of SPH simulations (e.g., [39]), or mesh cells in the case of moving mesh simulations (e.g., [46]), which appear capable of enriching diffuse environments in simulations without invoking superwind feedback (e.g., [107]). Tornatore et al. [108] tested the effect of the number of neighboring SPH particles on ICM properties, finding only moderate effects on the ICM metallicity. On the other hand, the Davé et al. [109] simulations spread metals over very few (i.e., 3) neighboring SPH particles, resulting in much lower IGrM oxygen metallicity arising from Type II SNe without mechanical feedback in contrast to their stellar feedback runs that have $\sim 1$ dex higher oxygen levels that better agree with observational constraints [110]. However, the Davé et al. [109] IGrM iron enrichment remains similar with and without stellar feedback, indicating enrichment from a different source-Type Ia $\mathrm{SNe}$ from older intracluster stars. Higher IGrM/ICM enrichment levels can also be achieved through increased numerical resolution as reviewed in Section 2.1.2 of Biffi et al. [111], owing primarily to the ability to resolve metal-enrichment from smaller halos at high-z.

While metal diffusion occurs naturally in mesh-based codes via advection between gas cells, metal diffusion between gas elements must be explicitly modeled in modern SPH implementations. The EAGLE simulations use SPH kernel-smoothed metallicities to calculate cooling rates [106] to estimate the dynamical effects of metal spreading in a manner that is consistent with the SPH formalism. The ROMULUS simulations apply the Shen et al. [112] metal diffusion algorithm to mimic turbulent diffusion based on the velocity shear between particles. Variations of metal mixing implementations are explored using GIZMO meshless finite mass codes in the FIRE-2 simulations [113] and the Rennehan [114] simulations, which found that the mixing algorithm significantly affects the resultant CGM and warm-hot IGM metallicity distributions.

In summary, the algorithm that describes how stars release metals into surrounding gas, the simulation resolution, and the metal mixing algorithm (if any is applied), are important considerations when simulating IGrM metallicities as discussed in the companion review by Gastaldello et al.[105].

\subsection{Stellar Feedback}

Stellar feedback, directly associated with stellar mass loss and enrichment, is discussed independently and is usually modeled by separate subgrid prescriptions. This owes to its uncertainty and range of possible outcomes that dramatically change the properties and 
appearances of galaxies in cosmological simulations (e.g., [62,115-119]). Stellar feedback has been identified as a key component of the overall solution to the overcooling problem whereby gaseous baryons are too efficiently converted into stars (e.g., [120]). Additionally, this feedback provides a pathway to enriching the high-redshift intergalactic medium (IGM; e.g., [121-126]), which is observed to have metal absorption far from the locations of galaxies (e.g., [127-131]). The enriched high- $z$ IGM certainly contributes to the IGrM metal content of simulated $z \sim 0$ groups that show enrichment levels consistent with observations (e.g., [109,132,133]).

Early cosmological simulations took the expected energy from Type II SNe and imparted velocity kicks to gas articles in star-forming regions [99]. Oppenheimer and Davé [134] applied a momentum-driven wind scaling [135] to their simulations to mimic the acceleration of dust-driven winds by stellar UV radiation pressure, resulting in greater mass-loading for lower-mass galaxies. The large range of stellar feedback prescriptions explored in the OWLS (OverWhelmingly Large Simulations; [116]) project, including varying the wind mass-loading (as a proportion of the SFR), wind velocity $\left(v_{\text {wind }}\right)$, and attempting thermally heated wind prescriptions, demonstrated very different outcomes for galaxies and gas.

Many modern cosmological simulations treat stellar feedback explicitly as a tunable subgrid model to reproduce key observations of galaxies including the galactic stellar mass function, galaxy sizes, and their central SMBH masses. Different physical motivations are used to justify parameter choices, but the resultant models are often far from the physical mechanisms of feedback. EAGLE tunes their feedback to heat SPH particles to $10^{7.5} \mathrm{~K}$ [39], justifying the choice of temperature to prevent catastrophic cooling and allow feedback to efficiently apply mechanical work [136]. IllustrisTNG uses a group finder to estimate the dark matter halo velocity dispersion $\left(\sigma_{\mathrm{DM}}\right)$ based upon Oppenheimer and Davé [137], and scale their kinetic wind velocities in proportion to $\sigma_{\mathrm{DM}}$ and the mass loading (i.e., the proportion of mass launched relative to SFR) in inverse proportion to $\sigma_{\mathrm{DM}}$ to a power that was calibrated [46]. SIMBA applies the wind velocity and mass loading scalings derived by Muratov et al. [138] from FIRE zoom simulations [139] that followed multiple sources of stellar feedback (radiation pressure, $\mathrm{SNe}$, stellar winds, and photo-ionization from stars) in their kinetic wind model [50].

The complexity of these modern subgrid stellar feedback prescriptions can be quantified in the amount of energy returned per unit of stellar mass formed. Davies et al. [140] calculated that EAGLE dumps $1.74 \times 10^{49} \mathrm{erg} \mathrm{M}_{\odot}^{-1}$ via thermal heating, and IllustrisTNG dumps $1.08 \times 10^{49} \operatorname{erg~}_{\odot}^{-1}$ with $90 \%$ going to a velocity kick and $10 \%$ going to thermal heating. This is the approximate energy injection rate expected if all SNe energy input into winds, that is, the expectation if there is $\sim 1 \mathrm{SN}$ with $10^{51}$ ergs per $100 \mathrm{M}_{\odot}$ formed. SIMBA limits their wind velocity to the total supernova energy available, which applies only to high- $z$ small galaxies. The ROMULUS simulations apply the Stinson et al. [141] 'blastwave' feedback model at an efficiency of $\approx 0.75$, with cooling temporarily disabled to prevent radiative losses.

It should also be noted that metal mass loading is treated differently than total mass loading in some simulations. Illustris and the TNG simulations apply reduced metal-loading that is $2.5 \times$ lower than mass-loading, because Vogelsberger et al. [90] argued that winds punch through low-density cavities in the ISM, reducing the metallicity of the ejected material. Alternatively, SIMBA applies higher metal loading that can be up to a factor of twice as high but is more typically $10 \%-20 \%$ higher.

While stellar feedback can reduce the efficiency of galaxy formation at lower masses, this mechanism becomes inefficient at preventing star formation in halos greater than $10^{12} \mathrm{M}_{\odot}$ (e.g., $[115,142,143])$, which is one of the key motivations for applying SMBH feedback.

\subsection{Black Hole Seeding}

Subgrid implementations of black hole (BH) seeding (Section 3.6), accretion (Section 3.7), and AGN feedback (Section 3.8) in simulations are introduced in Section 5.1 of the companion review by Eckert et al. [78], which we expand upon here with discussions of specific implementations applied in the simulations listed in Table 1. Most of these use a ver- 
sion of the Booth and Schaye [144] BH seeding module, which applies a friends-of-friends group finder to identify halos, originally performed by Di Matteo et al. [145], then seeding $10^{-3} M_{\text {gas }}$ sink particles in halos resolved with 100 dark matter particles (as in OWLS, Cosmo-OWLS, and BAHAMAS).

Illustris and EAGLE add $10^{5} \mathrm{~h}^{-1} \mathrm{M}_{\odot}$ BH seeds in $10^{10} \mathrm{~h}^{-1} \mathrm{M}_{\odot}$ halos, while IllustrisTNG uses larger seeds, $8 \times 10^{5} \mathrm{~h}^{-1} \mathrm{M}_{\odot}$ to avoid the need for boosted Bondi accretion (see next Section). SIMBA seeds $10^{4} \mathrm{~h}^{-1} \mathrm{M}_{\odot}$ BHs when $M_{*}>10^{9.5} \mathrm{M}_{\odot}$, skipping the attempt to follow $\mathrm{BH}$ accretion in low-mass halos. In general, dynamical friction is insufficiently resolved, therefore the default of these models is to continually re-position BHs to the local potential minimum (with the exception of Magneticum; see [146]).

The ROMULUS simulations instead seed SMBHs based on local gas properties: they can form whenever and wherever (i) gas density is 15 times the threshold for star formation; (ii) the local metallicity is low $\left(Z<3 \times 10^{-4}\right)$; and (iii) the temperature is just below the limit for atomic cooling. Consequently, SMBHs appear in ROMULUS at a higher redshift and in lower mass halos $\left(10^{8}-10^{9} \mathrm{M}_{\odot}\right)$ than in the other models discussed above. Moreover, SMBHs are not pinned to the local potential minimum; instead, the effects of unresolved dynamical friction are captured with a subgrid model [147]. This degree of freedom alters the SMBHs' growth and feedback trajectories $[48,49]$.

\subsection{Black Hole Growth}

Once seeded, black holes primarily grow through the accretion of gas, but can also grow through SMBH-SMBH mergers. For accretion, the most common implementation uses the Bondi-Hoyle accretion rate limited to the Eddington luminosity. As discussed in Section 5.1 of the companion review by Eckert et al. [78], initial implementations boosted the Bondi-Hoyle accretion rate by a large constant factor $(\approx 100)$ to compensate for the lack of dense gas in early low-resolution simulations [148]. Instead, Booth and Schaye [144] introduced a density-dependent boost to the Bondi-Hoyle accretion rate that only affects black holes surrounded by gas with an (unresolved) dense phase.

The Booth and Schaye [144] boost formula is used by many simulations, including cosmo-OWLS, BAHAMAS, Horizon-AGN, ROMULUS, while Illustris and FABLE use the older constant Springel et al. [148] boost. Magneticum uses a temperature-dependent boost [149] that increases for cooler gas to approximate turbulent-driven chaotic cold accretion rates [150]. (C-)EAGLE and IllustrisTNG forgo the need for boosted Bondi at their higher resolutions. The former modify their accretion rates by an additional viscous timescale to account for the angular momentum of infalling gas [151], with the intention to significantly reduce SMBH growth in low-mass halos. In this model, a longer viscous timescale due to high angular momentum of gas around the black hole delays accretion and translates into a higher $M_{\star}$ threshold where the $\mathrm{SMBH}$ accretion rate approaches the $\propto M_{\mathrm{BH}}^{2}$ Bondi limit ([62], but see [143]). A similar motivation is used in ROMULUS to modify the Bondi rate to account for gas rotation in addition to the relative velocity between the gas and the SMBH [48].

SIMBA calculates black hole accretion rates from cool gas with the 'torque-limited accretion' model [152,153], in an attempt to diverge from the self-regulated nature of Bondi accretion [144]. This model results in a much shallower dependence on $M_{\mathrm{BH}}$ than the Bondi formula [154], while also recognizing that there are preferred orientations for accretion in addition to feedback. Accretion from hot gas is modeled with the standard Bondi-Hoyle formula, but the torque-limited mode from cool gas generally dominates SMBH growth in SIMBA.

\subsection{AGN Feedback}

Groups provide some of the best evidence that AGN feedback significantly transforms the distribution of baryons, both in terms of the bulk transport of gas outward and the reduction in star formation [44,132]. Figure 1 demonstrates how adding AGN feedback using the Booth and Schaye [144] AGN prescription both reduces the gas fraction within $R_{500}$ and the luminosities of galaxies [132]. 

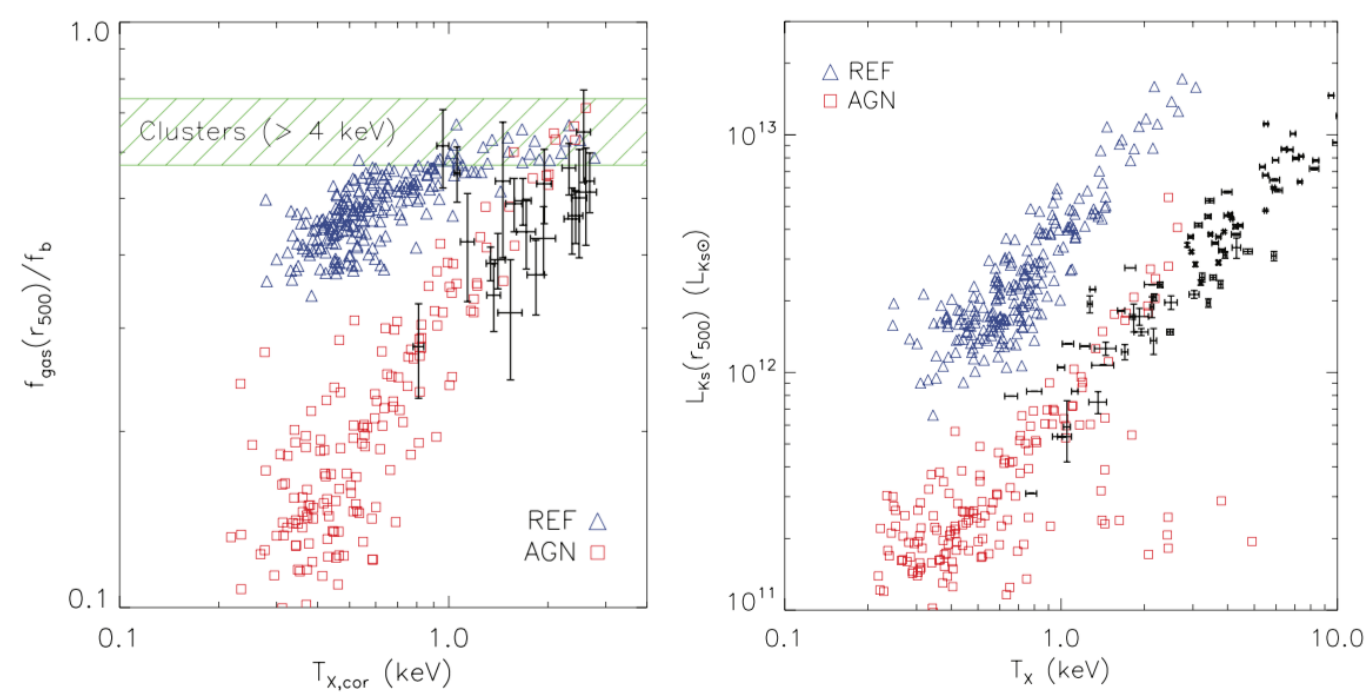

Figure 1. Figure panels adapted with permission from McCarthy et al. [132] showing OWLS simulations, with only stellar feedback (REF, blue triangles) and with additional AGN feedback (AGN, red squares). (Left) The AGN feedback provide a better fit to the Sun et al. [2] observationally derived $f_{500}$ data (black points) as a function of X-ray temperature; (Right) AGN feedback reduces the integrated K-band luminosity within $R_{500}$ to observable values compiled by Lin and Mohr [155].

Simulations usually apply an AGN feedback model that uses an AGN feedback efficiency term, $\varepsilon$, such that the feedback power is defined as $\dot{E}_{\mathrm{AGN}}=\varepsilon \dot{M}_{\mathrm{BH}} c^{2}$. Physically, $\varepsilon$ is a product of two efficiencies, the radiative efficiency $\left(\varepsilon_{r}\right)$, and the feedback efficiency $\left(\varepsilon_{f}\right)$. The radiative efficiency is the energy radiated away from the accretion onto the black hole, and is often assumed to be $10 \%$ based on the accretion onto a Schwarzschild BH [156]. The feedback efficiency is the fraction of the radiated energy that is imparted to the gas either thermally and/or kinetically.

A number of simulations that we list in Table 2 use a single-mode thermal model assuming $\varepsilon_{f}=0.15$ (i.e., resulting in $\varepsilon=0.015$ ), based on the value calibrated by Booth and Schaye [144] to reproduce the SMBH mass-halo mass relation derived from observations. The EAGLE Reference simulations apply a temperature increase of $\Delta T=10^{8.5} \mathrm{~K}$ to a neighboring SPH particle, once enough SMBH feedback energy has been accumulated to heat at least one SPH particle. The C-EAGLE/Hydrangea simulations run at the same resolution use a $\Delta T=10^{9.0} \mathrm{~K}$, which leads to less frequent, more bursty feedback that becomes more effective when heating gas above the virial temperatures of massive clusters [158]. The multiple cosmo-OWLS simulations varied $\Delta T$ while keeping $\varepsilon=0.015$ [7]. Their spatial resolution, nearly 1 dex lower than EAGLE, makes their heating temperature not directly comparable to the EAGLE $\Delta T$, since heating a single particle involves injecting a much larger amount of energy. In both cases, however, larger $\Delta T$ leads to a higher efficiency in transporting baryons beyond the virial radius. In BAHAMAS, feedback energy is accumulated until multiple gas particles can be heated simultaneously; McCarthy et al. [41] found that heating $20 \mathrm{SPH}$ particles $\left(\sim 2 \times 10^{10} \mathrm{M}_{\odot}\right)$ to $\Delta T=10^{7.8} \mathrm{~K}$, which was chosen as a calibration to prevent over-efficient assembly of intermediate-mass galaxies (see their Figure 3).

ROMULUS, with its higher resolution, uses a much lower efficiency $(\varepsilon=0.002)$, which was selected from a parameter search performed by Tremmel et al. [48] using galaxies in $10^{11-12} \mathrm{M}_{\odot}$ halos and not group/cluster-mass objects. Unlike stellar feedback, AGN feedback in ROMULUS is not subject to cooling shutoff.

Sijacki et al. [159] introduced the dual AGN model, with a "quasar" mode injecting thermal energy at high accretion rates, which is often defined as a relative fraction of the Eddington accretion rate, $f_{\mathrm{Edd}} \equiv \dot{m}_{\mathrm{Edd}} / \dot{m}_{\mathrm{BH}}$ where $\dot{m}_{\mathrm{Edd}}$ is the Eddington accretion limit, and a "radio" mode injecting mechanical energy in the form of bubbles at lower accretion rates. The radio mode (at low Eddington ratios) has more energy to impart 
into the gas since it bypasses the (inefficient) conversion from thermal to kinetic energy. Illustris switches to this mode when $f_{\text {Edd }}<0.05$, and releases built-up bubble events with sizes $\sim 100 \mathrm{kpc}$ when the $\mathrm{BH}$ grows by $15 \%\left(\delta M_{\mathrm{BH}}=0.15\right)$. The aggressiveness of this scheme over-evacuates the IGrM [160]. FABLE applied the same algorithm with similar energy efficiencies, but using smaller bubble events and a lower $f_{\text {Edd }}=0.01$ transition to radio mode that were the result of tuning their AGN feedback to reproduce IGrM gas fractions and galaxy stellar masses. Finally, more recent Magneticum simulations use the Steinborn et al. [149] thermal AGN feedback model that smoothly transitions between radio-mode and quasar-mode based on the fractional Eddington accretion rate.

Table 2. AGN feedback modules used in simulations.

\begin{tabular}{|c|c|c|c|c|c|c|}
\hline Simulation & Mode & Injection & Energy Dump & Efficiency $(\varepsilon)$ & Frequency & Loading Factor \\
\hline cosmo-OWLS & - & Thermal & $10^{8.0} \mathrm{~K}$ & 0.015 & Build-up & 1 particle \\
\hline Illustris & Quasar & Thermal & - & 0.01 & Continuous & \\
\hline- & Radio & Bubble & - & 0.07 & $\delta M_{\mathrm{B} H}=0.15$ & \\
\hline EAGLE & - & Thermal & $10^{8.5} \mathrm{~K}$ & 0.015 & Build-up & 1 particle \\
\hline Horizon-AGN & Quasar & Thermal & $10^{7} \mathrm{~K}$ & 0.015 & Build-up & \\
\hline - & Jet & Kinetic & $10^{4} \mathrm{~km} \mathrm{~s}^{-1}$ & 0.10 & Continuous & \\
\hline BAHAMAS & - & Thermal & $10^{7.8} \mathrm{~K}$ & 0.015 & Build-up & 20 particles \\
\hline $\begin{array}{l}\text { C- } \\
\text { EAGLE/Hydrangea }\end{array}$ & - & Thermal & $10^{9.0} \mathrm{~K}$ & 0.015 & Build-up & 1 particle \\
\hline ROMULUS & - & Thermal & - & 0.002 & Continuous & \\
\hline FABLE & Quasar & Thermal & - & 0.01 & $\delta t=25 \mathrm{Myr}$ & \\
\hline- & Radio & Bubble & - & 0.08 & $\delta M_{\mathrm{B} H}=0.01$ & \\
\hline IllustrisTNG & "High" & Thermal & - & 0.02 & Continuous & \\
\hline- & "Low" & Kinetic "Pulse" & - & $\leq 0.2$ & Build-Up & $\begin{array}{l}\text { Weinberger et al. [157] } \\
\text { Equation (13) }\end{array}$ \\
\hline SIMBA & "Radiative" & Kinetic & $1000 \mathrm{~km} \mathrm{~s}^{-1}$ & $0.003^{\mathrm{a}}$ & Continuous & \\
\hline- & "Jet" & Kinetic "Jet" & $8000 \mathrm{~km} \mathrm{~s}^{-1}$ & $0.03^{\mathrm{a}}$ & Continuous & \\
\hline
\end{tabular}

${ }^{\text {a }}$ Values for a $10^{9} \mathrm{M}_{\odot} \mathrm{SMBH}$.

Several simulations attempt to simulate jets or jet-like feedback at low Eddington rates. Horizon-AGN uses the Dubois et al. [161] bipolar kinetic jet aligned with the spin of the SMBH. IllustrisTNG integrates the Weinberger et al. [157] model, where randomly-oriented, directional kinetic "pulses" predominantly take over once a SMBH grows above a certain mass based on their Equation (5). In practice, this choice yields significant implications as TNG100 SMBHs growing above a threshold mass of $M_{\mathrm{SMBH}} \approx 10^{8.1} \mathrm{M}_{\odot}$ at late times leads to a sharp transition of galaxy properties (reduced SFRs, quenched galaxies, redder colors) and gaseous halo properties (lower $f_{\text {gas }}$ ) [162,163]. Low Eddington ratio feedback in SIMBA uses a bipolar kinetic jet perpendicular to the angular momentum of the local disc that has a constant momentum input rate $(20 \times$ the radiative luminosity of the accretion disc divided by the speed of light). This is meant to complement the preferred orientation of the Davé et al. [50] torque-limited accretion model. This model also decouples for $10^{-4}$ of a Hubble time, which can transport launched jet particles over $\sim 10 \mathrm{kpc}$.

\subsection{Transport Processes and Magnetic Fields}

It is fair to say that the effects of viscosity, thermal conduction, and turbulence (which we collectively refer to as 'transport processes') on the IGrM have received significantly less attention in comparison to, for example, the overall gravitational and hydrodynamical evolution of groups and the impact of processes associated with galaxy formation (such as radiative cooling and feedback processes). Indeed, from the cosmological simulations standpoint, the vast majority of existing simulations completely neglect the roles of viscosity and conduction (treating the IGrM as an inviscid and non-conducting fluid), while the effects of turbulence are generally only captured on relatively large, well-resolved scales.

However, there are no a priori compelling physical reasons for neglecting these processes, as the IGrM and the ICM are both plasmas where, generally speaking, one expects such transport processes to be active [164]. To include their effects in cosmological simulations is non-trivial, though, as the hydro solvers that normally evaluate the standard (inviscid) hydrodynamic equations must be replaced with more complex solvers capable of 
evaluating the full Navier Stokes equations in a stable fashion, at least if one wishes to model the effects of viscosity (e.g., [165]). Furthermore, since the transport of heat by viscosity and conduction preferentially occurs along magnetic field lines (and is strongly suppressed perpendicular to the field lines), it really only makes sense to include their effects in the context of MHD simulations that self-consistently follow the evolution of the magnetic fields. While there is a growing interest in the inclusion of anisotropic thermal conduction, viscosity and magnetic fields in simulations (e.g., $[55,166])$, we are presently unaware of any large-scale cosmological simulations that include both and have evaluated their impacts on the plasma in groups and clusters ${ }^{8}$. In terms of modeling turbulence, modern hydrodynamical solvers accurately follow the cascade of energy, momentum, and mass down to the effective resolution scale of the simulations but generally not below this. Thus, there are ongoing efforts to model the turbulent cascade to smaller scales using physically-motivated subgrid models (e.g., [112,168]). Whether turbulence plays a large role or if viscosity is able to strongly damp the cascade depends on the dimensionless Reynolds number but, at present, this is a very poorly constrained quantity for the IGrM and ICM.

What impact might we expect from including anisotropic conduction, viscous heating and/or small-scale turbulence in simulations of the IGrM? After the launch of Chandra there was much excitement with the discovery of large bubbles of relativistic plasma that appear, at least in some cases, to remain structurally intact even after buoyantly rising to relatively large distances from their inflation points (e.g., [169]). This is difficult to understand from an unmagnetized, inviscid (and therefore highly turbulent) fluid standpoint, as the bubbles should have been rapidly destroyed/mixed via Kelvin-Helmholtz and Rayleigh-Taylor instabilities. High-resolution idealized simulations of viscous and/or magnetized clusters, however, demonstrated that the bubbles were much more structurally stable and longlived when these processes were included (e.g., $[170,171])$. Thus, how and where bubbles transmit their energy to the IGrM will likely be impacted by the inclusion of transport processes and magnetic fields. In addition, damping of sound waves (e.g., produced during the inflation of bubbles) via the viscous friction has been proposed as another way in which the central AGN might couple its energy to the gas over a large volume (e.g., [172,173]). The evolution of satellite galaxies (i.e., when and how fast they are stripped) may also be strongly affected by the inclusion of conduction, viscosity, magnetic fields and/or small-scale turbulence (see, e.g., Figure 8 of [165] for a dramatic demonstration of viscous stripping in clusters). While, theoretically, we expect thermal conduction and viscous dissipation to be considerably more important in massive clusters compared to groups, as the transport coefficients have strong temperature dependencies, much depends on the geometry of the magnetic field lines and, at present, the impact of these transport processes on the evolution of the IGrM and the satellites in groups remains an important unknown. The advent of new codes such as AREPO and GIZMO that are capable of accurately incorporating their effects is a promising step in the right direction and we expect to see important progress in answering these questions in the coming years.

\subsection{Cosmic Rays}

Although cosmic ray (CR) physics has not yet been included in cosmological simulations of groups or clusters, recent advancements in CR hydrodynamics and results from both idealized simulations of massive galaxies and cosmological simulations of lower mass galaxies demonstrate that cosmic rays may be an important source of pressure and energy in galaxy groups. In this subsection we briefly describe existing approaches to modeling CR physics and their expected effects in galaxy groups.

In hydrodynamics galaxy simulations, cosmic rays are typically modeled as a relativistic fluid of GeV protons, separate from the thermal gas [174-176]. This CR fluid advects with the gas and can provide non-thermal pressure support, inject momentum, or heat the gas. Additionally, cosmic rays can move relative to the gas through diffusion or streaming, both of which are approximations of the bulk flow of CR energy density along magnetic field lines (see $[177,178]$ for a comprehensive review). Unfortunately, there is no empirical 
or theoretical consensus on the CR pressure in galaxy groups or on the correct model for $\mathrm{CR}$ hydrodynamics. For this reason, quantitative predictions of galaxy, outflow, and halo properties from simulations can vary by orders of magnitude depending on the model parameters [179-181]. However, there are several qualitative ways in which cosmic rays alter galaxy and halo properties that are consistently demonstrated in simulations.

(1) Cosmic ray transport (either streaming or diffusion) can drive galactic outflows [182-186]. Cosmic rays are injected into the ISM during stellar feedback events (typically $~ 10 \%$ of the total supernova energy). CR transport redistributes CR pressure out of the galaxy, creating a non-thermal pressure gradient that exerts a force opposing gravity. If the force exerted by the $\mathrm{CR}$ pressure gradient is sufficiently strong, it will trigger galactic outflows. Relative to thermally driven winds, CR driven winds are cooler, smoother, and more mass-loaded [187]. Additionally, since cosmic rays do not suffer radiative losses, CR-driven winds may continue accelerating gas at large distances from the galactic disc. However, since the gravitational force is stronger in more massive galaxies, CR-driven winds may become inefficient in galaxy groups [188].

(2) Streaming cosmic rays impart energy to heat the surrounding gas. In massive galaxies, this CR heating rate can efficiently balance radiative cooling, preventing a cooling catastrophe (e.g., [189-192]). CR heating may also be a key aspect in the self-regulated AGN feedback cycle. As cosmic rays lose energy, gas cools more efficiently, fueling AGN feedback which re-injects CR energy into the IGrM [193].

(3) CR pressure qualitatively alters the structure of multiphase gas in galactic halos (e.g., [180,194-196]). Non-thermal pressure support enables cool gas to exist at lower densities than expected from purely thermal equilibrium. Figure 2 demonstrates how the density contrast between cold and hot gas in a two-phase medium diminishes with increasing CR pressure support. In the extreme case of a CR pressure-dominated galaxy halo, cool and hot gas can exist at the same densities. However, CR pressure is unlikely to be the dominant source of pressure in the halos of massive galaxies. Therefore, the likely effect of cosmic rays in the IGrM is a modest decrease of cool cloud and cool filament densities [197,198].

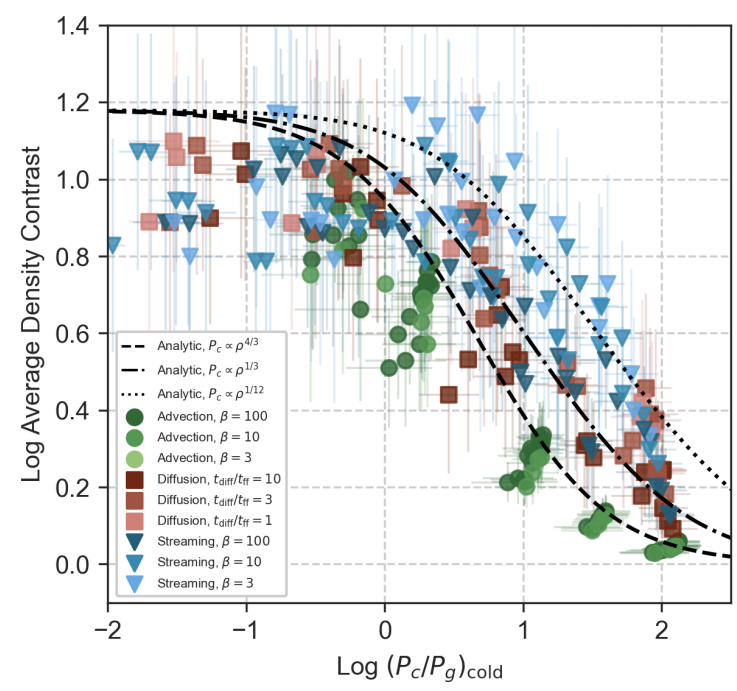

Figure 2. The average density contrast between cold and hot gas phases as a function of cosmic ray pressure support in the cold gas [199]. Each point represents a time-averaged measurement from an idealized simulation of thermal instability with varying initial conditions and cosmic ray physics. The black lines show different analytical predictions for various degrees of coupling between cosmic rays and gas. With increasing cosmic ray pressure, the density of cool gas decreases. However, the detailed quantitative predictions are sensitive to the invoked cosmic ray transport model. 


\section{Results of Simulations at the Group Scale}

\subsection{The Baryonic Content of Group Halos}

The intermediate scale of group halos between galactic halos and clusters provides a unique lever arm on the nature of superwind feedback. Unlike most galactic halos, the temperature, density, and metallicity of their gaseous baryons can be probed via soft X-ray emission without stacking, while their shallower gravitational potential wells relative to clusters allow energetic feedback to remove a significant fraction of baryons. Twenty years ago, IGrM observations from missions including ROSAT provided only singular data points per group, which were then compiled into the $L_{X}-T_{X}$ relation. The steep $L_{X} \propto T_{X}^{4.9}$ relation observed for groups [200] relative to clusters [201] indicated a significant deviation from the $L_{X} \propto T_{X}^{2}$ relationship expected for virialized gaseous halos retaining all their baryons in hydrostatic equilibrium (e.g., [202], see companion review by Lovisari et al. [1]).

With the launch of more powerful X-ray missions, such as Chandra and XMM-Newton, hot gas profiles around groups could be resolved. This revealed that the IGrM had far fewer gaseous baryons than the cosmic ratio, $f_{\mathrm{b}} \equiv \Omega_{\mathrm{b}} / \Omega_{\mathrm{M}} \simeq 0.16$, inside $R_{2500}$ and a still low proportion inside $R_{500}$ [2,5]. Altogether these results indicated that many of the baryons are removed from the hot phase. Earlier theoretical work argued that more efficient line cooling at cooler group temperatures, as opposed to clusters where Bremsstrahlung cooling dominates $\left(\Lambda_{\text {cool }} \propto T^{0.5}\right)$, could more efficiently build the stellar component of galaxies [203,204]. While Gonzalez et al. [205] measured that the integrated group stellar masses from the BGG, the intragroup light (IGrL), and satellite galaxies could account for the missing gaseous baryon resulting in a groups retaining all baryons inside $R_{500}$, this was later challenged. Balogh et al. [206] argued that groups could not be far more efficient than clusters in converting their gas to stars due to the hierarchical requirement that clusters are assembled from progenitor groups. More recent compilations of group stellar masses summed from BGGs, satellites and IGrL [207] find lower stellar mass fractions, strongly suggesting that groups are missing baryons.

Cosmological volume simulations with periodic volumes 100-150 comoving Mpc on a side contain populations of groups that can be statistically compared to observations. Initial simulations by Davé et al. [109], including only stellar superwind feedback, were able to enrich the IGrM to observed levels [200] and additionally add entropy to group halos as observed [208]; however the continued late-time star formation in these groups is a telltale sign that these simulations fail to solve the cooling flow problem [209]. Liang et al. [37] used GADGET-2 SPH simulations similar to Davé et al. [109] to successfully fit a range of X-ray observations, including IGrM masses inside $R_{500}$. Nevertheless, the total stellar masses exceeded observations by at least a factor of two, and the total baryon content (gas+stars) of groups exceeded $80 \%$ of the cosmic fraction. These simulations demonstrated that it is possible to reproduce a wide range of IGrM properties, while assembling the wrong galaxies, which in these cases had too much late-time star formation.

McCarthy et al. [132] made the case for AGN feedback by using simulations from the OWLS [116] suite of simulations by comparing to Chandra observations from Sun et al. [2] that resolved X-ray emission profiles out to $R_{500}$ in group-scale objects. Comparing OWLS simulations without and with Booth and Schaye [144] AGN feedback, McCarthy et al. [132] demonstrated that the latter could much better reproduce $f_{\text {gas }}\left(R_{500}\right)$, the $L_{X}-T_{X}$ relation, as well as the stellar K-band luminosity of the BGG and all stars within $R_{500}$ for the approximately 200 group-sized objects in $100 \mathrm{~h}^{-1} \mathrm{Mpc}$ boxes with gas element resolution of $1.2 \times 10^{8} \mathrm{M}_{\odot}$ (see Figure 1). We refer the reader to the Eckert et al. [78] companion review (their Section 5.2) for a discussion of a broader range of simulations and their baryon fractions within $R_{500}$. 


\subsubsection{Gaseous and Stellar Masses in Recent Simulations}

One can consider as "contemporary" intermediate resolution simulations those that resolve gas resolution elements at $\sim 10^{6} \mathrm{M} \odot$ mass resolution in $\sim 10^{6} \mathrm{Mpc}^{3}$ volumes. The first simulations to satisfy these criteria were Illustris [38] and EAGLE [39]. Both simulations follow SMBH growth and AGN feedback, and are tuned to match the $z=0$ stellar mass function, some other galaxy characteristics such as galaxy sizes, and SMBH demographics. However, they both were not tuned to reproduce the properties of X-ray emitting halos, and fail to reproduce gaseous properties of groups and poor clusters. Genel et al. [160] showed that Illustris severely underpredicts the IGrM mass within $R_{500}$, by as much as a factor of $10 \times$ compared to observations by Giodini et al. [210]. EAGLE produces group halos with much higher gas fractions, exceeding $80 \%$ of $f_{\mathrm{b}}$ at $M_{200}>10^{13.5} \mathrm{M}_{\odot}$ [211]. We plot gas, stellar, and baryon fractions inside $R_{200}$ as a function of $M_{200}$ in Figure 3 for EAGLE and other contemporary simulations ${ }^{9}$. Schaye et al. [39] found the IGrM masses derived from virtual X-ray observations to be too high by a factor of two and the $L_{X}-T_{X}$ relationship to be too luminous for a given $T_{X}$. This reveals that simulations tuned to fit galaxies can deviate significantly for gaseous properties on group scales, which is why accurately simulating the IGrM can provide orthogonal constraints on the processes governing galaxy formation and evolution.

BAHAMAS explicitly tuned their AGN feedback prescriptions to reproduce properties of groups/clusters and massive galaxies as explained in Section 3.8. The FABLE simulations ([44], not shown in Figure 3) also explicitly tuned their feedback to reproduce massive halos, at $\sim 100 \times$ higher mass resolution than BAHAMAS but in a $1000 \times$ smaller volume that is augmented by a series of zooms extending up to cluster masses. These simulations have $f_{\text {gas, } 500}$ for a $M_{500}=10^{13.5} \mathrm{M}_{\odot}$ between 0.04 and 0.07 in Table 1 , which reflects the uncertainty in the Sun et al. [2] and Lovisari et al. [5] observations (see Figure 5 of the companion review by Eckert et al. [78]).

The C-EAGLE/Hydrangea zooms [42,43] use the EAGLE model with a higher AGN heating temperature $\left(\Delta T=10^{9.0} \mathrm{~K}\right)$. As shown by Schaye et al. [39], this change lowers (i.e., improves) $f_{\text {gas, } 500}$ in $M_{500} \lesssim 10^{13.5} \mathrm{M}_{\odot}$ groups, but more massive objects remain too baryon rich, especially in the regime of rich groups/poor clusters [43]. Similarly, the IllustrisTNG AGN feedback was calibrated to $f_{\text {gas, }}$ 500 of groups at TNG100 resolution [157], though the simulations still predict $f_{\text {bar, } 200} \gtrsim 0.12$ for halos with $M_{200}>10^{13.5} \mathrm{M}_{\odot}$, substantially higher than the explicitly calibrated BAHAMAS simulation (see the right-hand panel of Figure 3). SIMBA [50] predicts $f_{\text {gas,200 }} \approx 0.05$ at $M_{500}=10^{13.5} \mathrm{M}_{\odot}$ and agrees well with BAHAMAS in this metric within the overlapping halo mass range. Interestingly, the companion review by Eckert et al., however, shows that $f_{\text {gas,500 }}$ in SIMBA increases more rapidly with halo mass in SIMBA compared to BAHAMAS (their Figure 15), so that more baryons are contained near the center of poor clusters with $M_{500}=10^{14-14.5} \mathrm{M}_{\odot}$. We will return to the importance of the radial range for assessing the baryon content of simulated groups below.

Higher resolution simulations $\left(m_{\mathrm{bar}} \sim 10^{5} \mathrm{M}_{\odot}\right)$ currently contain at best a handful of group halos, but still yield constraining results. The ROMULUS suite [48,49] predicts $f_{\text {gas }, 200}=0.10-0.12$ between $M_{200}=10^{12.5-14.0} \mathrm{M}_{\odot}$ and a higher $f_{*, 200}$ than all the aforementioned simulations (orange lines in Figure 3). These halos therefore retain nearly all their baryons, in strong conflict with the observational evidence outlined above. It is unlikely that this shift is a direct consequence of the higher resolution, since the higherresolution simulation of the IllustrisTNG family, TNG50 [51], predicts $f_{\text {gas,500 }}$ rising from 0.06 to 0.12 over the mass range $M_{500}=10^{13.0-14.0} \mathrm{M}_{\odot}$ (not shown), in excellent convergence with TNG100 (we find a similar result for the lower-resolution version, TNG300). 

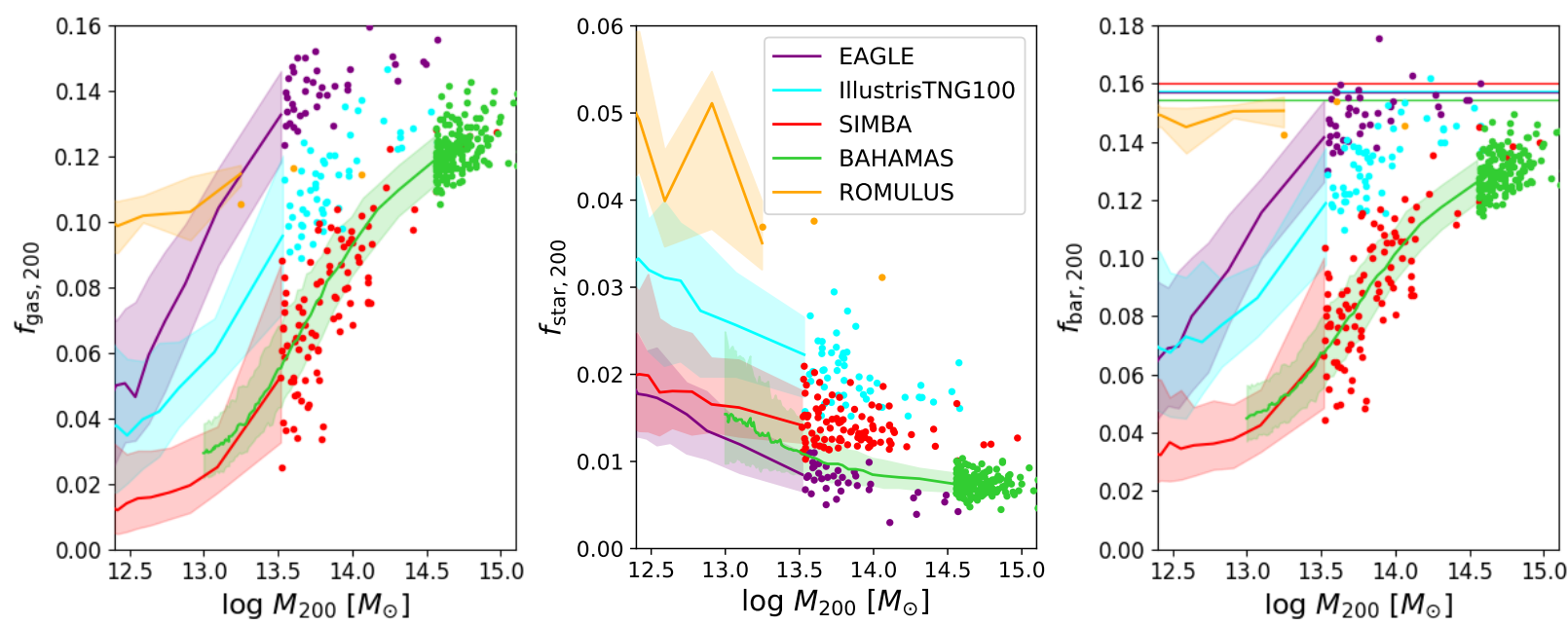

Figure 3. Gas, stellar and combined baryon fractions inside $R_{200}$ from a variety of widely-used, contemporary cosmological hydrodynamic simulations. Medians and 1- $\sigma$ spreads are shown with lines and shading up to a mass where individual objects are plotted as points. The cosmic baryon fraction $f_{b}=\Omega_{\mathrm{b}} / \Omega_{\mathrm{M}}$ is plotted in the right-hand panel for the given simulation cosmology. IGrM measurements rarely extend to $R_{200}$, therefore this plot does not include observations. It is notable that stellar contents do vary by a factor of more than $3 \times$ between different simulations in the group regime, and more than double between EAGLE and IllustrisTNG. ROMULUS retains almost all of its baryons within $R_{200}$ while BAHAMAS and SIMBA eject more than half their baryons at $M_{200} \lesssim 10^{13.5} \mathrm{M}_{\odot}$. For reference, $M_{500}$ is typically 0.16 dex lower than $M_{200}$ for this halo mass range.

The determination of IGrM properties within $R_{500}$ is physically motivated, but measurements within fixed apertures are observationally more straightforward. In Figure 4, we therefore show the integrated IGrM masses as a function of halo mass as predicted by EAGLE, SIMBA, and the three IllustrisTNG boxes out to fixed radii of 100, 200, and $400 \mathrm{kpc}$ in the 3 subpanels. For comparison, we obtain the equivalent masses from the observations of Sun et al. [2] and Lovisari et al. [5] by integrating their measured electron density profiles out to the same radii. While the relative differences between the simulations shown in Figure 4 are consistent across the three radial cuts-remarkably close agreement between the three TNG runs, with EAGLE and SIMBA offset by $\approx+0.1$ and -0.5 dex, respectively, at $M_{500}=10^{13.5} \mathrm{M}_{\odot}$ 一the comparison to the observations reveals additional details in each panel. Close to the group center $(r \leq 100 \mathrm{kpc})$, EAGLE shows promising agreement with the observations, owing to its comparatively less aggressive AGN feedback. SIMBA, on the other hand, despite having calibrated its AGN model to match the IGrM fraction inside $R_{500}$, is evacuating the central region too efficiently. Within the larger $400 \mathrm{kpc}$ aperture (right-hand panel), the IGrM masses of EAGLE are higher than observed, while SIMBA is at least marginally consistent with the observations at the high-mass end $\left(M_{500} \gtrsim 10^{14} \mathrm{M}_{\odot}\right)$. The IGrM masses of IllustrisTNG are consistent with the observations across radii, albeit with a tendency of being too low in the center $(r<100 \mathrm{kpc})$ of low-mass groups $\left(M_{500} \lesssim 10^{13.5} \mathrm{M}_{\odot}\right)$. 

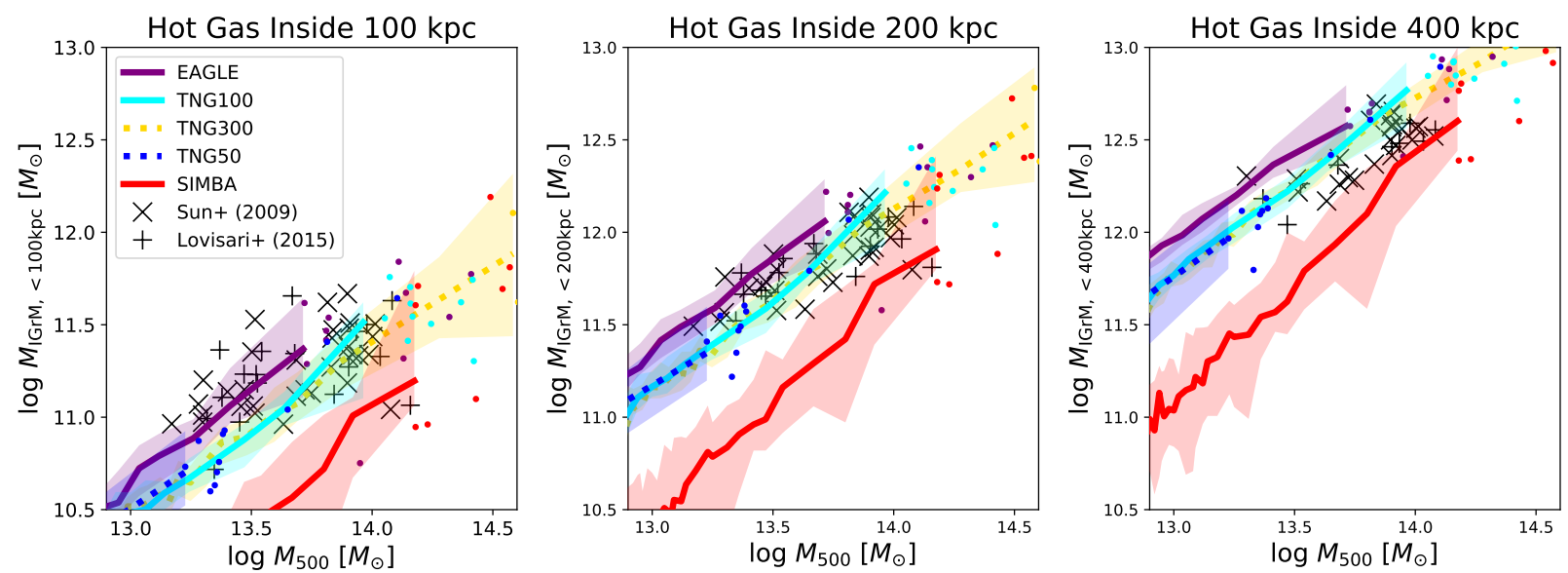

Figure 4. IGrM masses (gas at $T \geq 10^{6} \mathrm{~K}$ ) within three fixed 3D apertures plotted for EAGLE (purple), SIMBA (red), and all three IllustrisTNG volumes (dark blue, cyan, and yellow). Medians and $1 \sigma$ scatter are indicated by lines (dotted for TNG50 and TNG300, solid for others) and shaded regions, respectively. Different panels correspond to limiting radii of $100 \mathrm{kpc}$ (left), $200 \mathrm{kpc}$ (middle), and $400 \mathrm{kpc}$ (right). Observed IGrM masses of individual groups within these radii, obtained from the (3D) profiles of Sun et al. [2] and Lovisari et al. [5], are shown as black symbols. While differences between simulations are approximately consistent across the three limiting radii, the agreement with observations differs noticeably between the group center (left-hand panel) and outskirts (right-hand panel).

\subsubsection{Gaseous Profiles in Recent Simulations}

We have seen above that even simulations that were calibrated to match gas and stellar fractions within the virial radius can make discrepant predictions about the IGrM content within other radii. A more challenging test of the simulations is therefore provided by their predicted IGrM profiles, which are plotted for $z \approx 0$ groups of $M_{500}=10^{13.5}-10^{14.0} \mathrm{M}_{\odot}$ from EAGLE, TNG100, SIMBA, and ROMULUS ${ }^{10}$ in Figure 5. In the top left panel, we show stacked profiles of electron density $n_{\mathrm{e}}$, calculated as $n_{\mathrm{e}}=\rho_{\mathrm{IGrM}} /\left(\mu_{\mathrm{e}} m_{\mathrm{H}}\right)$ with $\rho_{\mathrm{IGrM}}$ the density of the hot IGrM gas (here defined as $T>10^{6} \mathrm{~K}$ ), $\mu_{\mathrm{e}}=1.14$ the mean molecular weight per free electron, and $m_{\mathrm{H}}$ the proton mass. Stacked IGrM temperature profiles are shown in the top-right panel. In both cases, we compare to observed profiles of individual groups in the same mass range from thin dashed lines color-coded by mass (Sun et al. [2]); for density we also plot the stacked observed profile of black dash-dotted lines (Lovisari et al. [5]).

All simulations (except ROMULUS, which may be affected by its small sample size) agree closely with each other for the temperature profile beyond $\approx 0.1 R_{500}$, and are broadly consistent with the observations of Sun et al. [2]. This similarity is interesting in the context of AGN feedback prescriptions that heat the gas differently resulting in similar temperature profiles throughout most of the IGrM, indicating that virialization primarily sets the IGrM temperature. Significantly more variety is seen in the density profiles (top-left); all simulations predict a comparable and realistic density around $R_{500}$, the increase towards smaller radii is substantially stronger for ROMULUS, and weaker for SIMBA, than observed. TNG100 and EAGLE, on the other hand, agree quite closely with each other and the observations, albeit with a slight $(\leqslant 0.2$ dex) excess of gas towards the outskirts-especially for EAGLE— and a more substantial deficit $(\approx 0.2-0.5$ dex $)$ near the center that is stronger for TNG100.

Of particular interest are two physically motivated combinations of density and temperature: the IGrM pressure $P \equiv n_{\mathrm{e}} T$ and its entropy $K \equiv T /\left(n_{\mathrm{e}}^{2 / 3}\right)$. These not only highlight additional discrepancies between simulations and observational data, but reveal imprints of the subgrid prescriptions, most notably AGN feedback schemes. We show their radial profiles, normalized to their analytically expected values within $R_{500}$ : for pressure, $P_{500} \equiv k_{\mathrm{B}} T_{500} n_{\mathrm{e}, 500}$ and for entropy, $K_{500} \equiv k_{\mathrm{B}} T_{500}\left(n_{\mathrm{e}, 500}\right)^{-2 / 3}$, where $T_{500}$ is taken as the virial temperature $k_{\mathrm{B}} T_{500} \equiv G M_{500} \mu m_{\mathrm{H}} / R_{500}$ with mean molecular weight $\mu=0.59$ and the electron density $n_{\mathrm{e}, 500}$ as the ideal value of $n_{\mathrm{e}, 500} \equiv 500 f_{\mathrm{b}} \rho_{\text {crit }} /\left(\mu_{\mathrm{e}} m_{\mathrm{H}}\right)$ in the bottom row of Figure 5. 

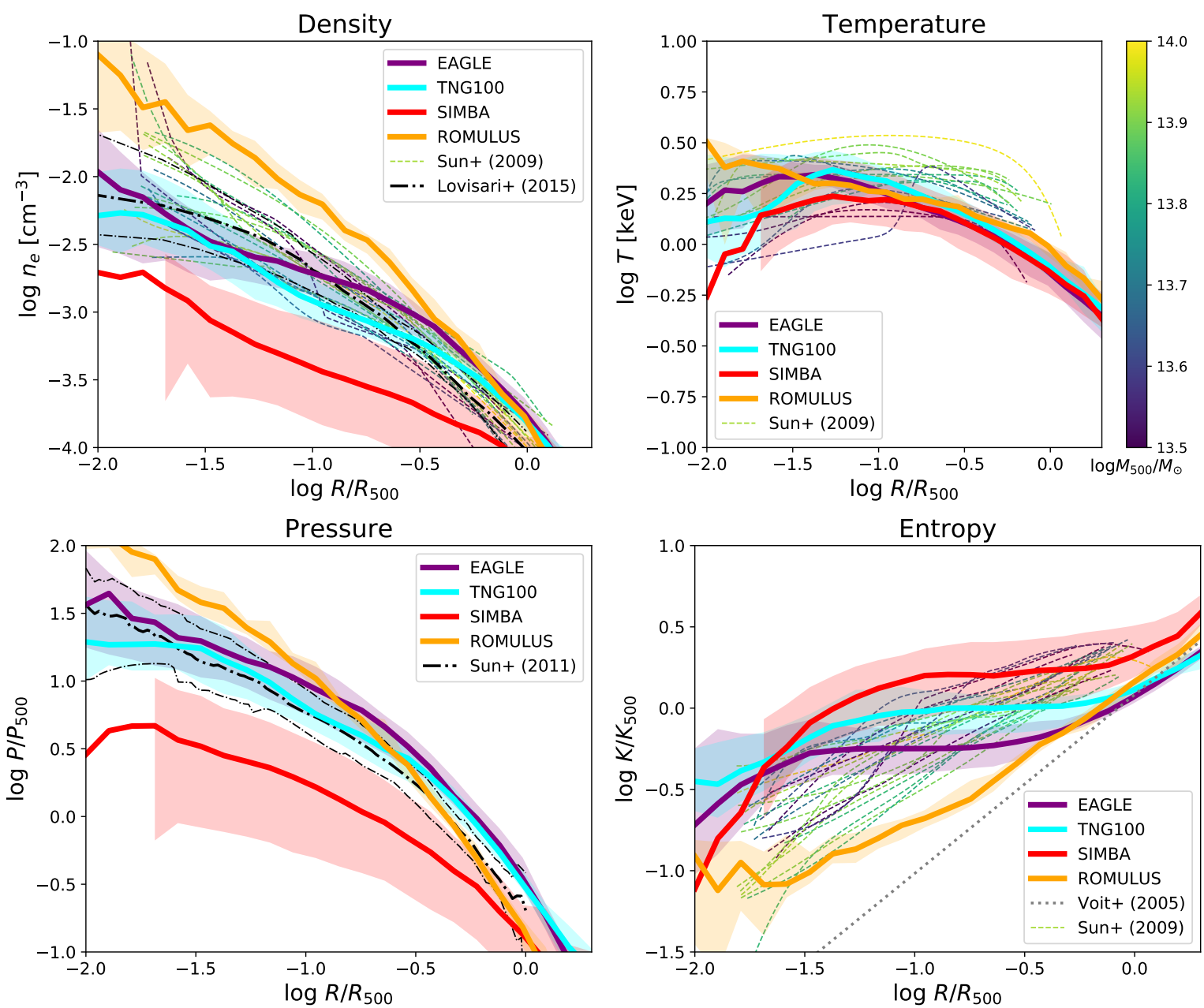

Figure 5. Mass-weighted 3D radial profiles of IGrM density (top left), temperature (top right), pressure (bottom left) and entropy (bottom right) in groups with $M_{500}=10^{13.5}-10^{14.0} \mathrm{M}_{\odot}$ from the EAGLE $(N=18$ groups, purple), TNG100 ( $N=35$, cyan), SIMBA ( $N=80$, red), and ROMULUS ( $N=1$, orange) simulations. For the first three, we show profiles at $z=0$, while for ROMULUS we have stacked the profiles obtained from the single group in this mass range at five snapshots with $z \leq 0.36$. Running medians within each simulation are plotted as solid lines, with shaded bands representing the $1 \sigma$ scatter. For comparison, profiles of individual groups in the same mass range derived from X-ray observations [2] are shown as thin dashed lines in three panels, colored by (temperature-derived) halo mass; for pressure, we instead show the SZ-based profiles of the same groups by Sun et al. [212] as black dash-dotted lines (thick and thin ones for the median and $1 \sigma$ scatter, respectively). In the top-left panel, the stacked density profile from X-ray observations of Lovisari et al. [5] is shown in the same fashion, while the grey dotted line in the bottom right panel represents the "base line" $K \propto r^{-1.1}$ entropy profile seen in non-radiative simulations (e.g., Lewis et al. [95], Voit et al. [96]). With the exception of temperature at large radii, there is little agreement between simulations. The entropy profiles in particular are also clearly different from what is observed in all four cases, even for simulations such as EAGLE and TNG100 that approximately reproduce the observed pressure profile. In general, stronger AGN feedback prescriptions raise entropies and reduce densities and pressures.

At large radii, all simulations follow a power-law entropy profile with an index close to 1.1, as expected from accretion and associated shocks [213] and as found in non-radiative simulations (e.g., $[95,96]$ ). Within $\approx 0.3 R_{500}$, the median profiles of three simulations (SIMBA, TNG and EAGLE) flatten to an extended entropy core, while ROMULUS entropy profiles keep decreasing and only flatten in the very center. The magnitude of these simulated entropy cores appears to scale broadly with the aggressiveness of the AGN feedback. A factor of 100 difference in the energy coupling efficiency $\varepsilon$ of AGN feedback progressively raises the entropy levels from ROMULUS $(\varepsilon=0.002)$ to TNG $(\varepsilon=0.2$ for their pulse mode). SIMBA, however, exemplifies that the situation is more complex; it has the highest entropy core levels ( $\gtrsim K_{500}$ beyond $\left.0.03 R_{500}\right)$ despite an AGN coupling 
efficiency comparable to EAGLE (see Table 2). It is plausible that the high entropy core of SIMBA is, at least in part, due to its decoupled kinetic AGN feedback scheme [50], which can efficiently inject entropy at large radii. At the same time, it still allows transport of low-entropy gas towards the center, leading to the strong drop in central entropy (which, to a lesser extent, is also seen for EAGLE).

Although the core entropy levels in TNG100 and EAGLE are lower than for SIMBA, their AGN feedback schemes - randomly-oriented, pulsed feedback [157] and highly energetic thermal injection [39], respectively—are still creating extended cores. The only simulation without a clear high-entropy core is ROMULUS, which injects AGN energy thermally but with a much lower temperature increase. As we have seen above, this low entropy injection correlates with higher group baryon fractions than in the other simulations.

A comparison of these predictions to the Sun et al. [2] profiles clearly reveals that none of the scaled entropy profiles from any of the simulations resemble the observations. The latter do not have large extended plateaus; they typically scale with radius as $R^{0.7}$ for $R<R_{500}$ (see also Figure 10 of Sun et al. [2] and Figure 4 of O'Sullivan et al. [8] for the CLoGS group sample). This is despite reasonable agreement in terms of the pressure profiles (especially for EAGLE and TNG100), which indicates that the simulated IGrM remains approximately in pressure equilibrium throughout the (significant) AGN energy and entropy injection [43]. The FABLE simulations (not shown [44]) show more promising agreement of IGrM profiles (including entropy), although Henden et al. [44] discuss that even their explicitly calibrated (bubble) feedback might be too energetic at late times, since the $z=0$ FABLE groups fall within the scatter, but mostly below the median, of the Sun et al. [2] density profiles (see their Figure 11). It will be interesting to see whether future simulations can overcome this shortcoming with more sophisticated AGN feedback models and higher resolution, or whether observational selection biases (i.e., preferential inclusion of cool-core systems with dense, bright centers in X-ray selected samples as discussed by, e.g., Henden et al. [44]) are responsible for at least part of the discrepancy (see Section 5).

For deeper insight into the predicted IGrM entropy profiles, it is instructive to consider them in context with their more massive cluster counterparts. This comparison is shown in the left panel of Figure 6, where we plot scaled $z \approx 0$ entropy profiles in analogy to the bottom-left panel of Figure 5 but over a wide range of halo mass, $M_{500}=10^{13.0-15.0}$. For clarity, only IllustrisTNG (TNG100 and TNG300 combined, with TNG300 dominating because of its larger volume; solid lines) and ROMULUS (dashed lines) are shown, with halos median-stacked within 0.5 dex bins in $M_{500}$. These are compared to three observational samples that together span a similar mass range: the Sun et al. [2] and (lower-mass) CLoGS [8] groups as well as clusters from the ACCEPT survey [9].

There are two features of this comparison that are particularly worth highlighting. First, the median entropy profiles of IllustrisTNG feature prominent entropy cores that are significantly higher than observed across the selected mass range, from poor groups (purple) to massive clusters (yellow). The same is true for EAGLE and SIMBA clusters (not shown). On cluster scales, these high-entropy cores corresponding to non-cool-core (NCC) systems have previously been highlighted by C-EAGLE (Barnes et al. [43]), IllustrisTNG (Barnes et al. [214]), and SIMBA (Robson and Davé [215]); in the case of IllustrisTNG, Barnes et al. [214] found a rapid decline in the fraction of cool-core (CC) systems at $z<1$ and identified the cause to be the AGN feedback implementation, which appears too efficient at removing baryons from the inner $0.01 R_{500}$.

Second, there is a clear trend towards lower (normalized) entropy with increasing halo mass at fixed radius, in both the observations and the IllustrisTNG simulations ${ }^{11}$. We speculate that these features may be a signature of quantized feedback dumping a fixed amount of entropy per AGN feedback event; further investigation of this topic is clearly warranted. 

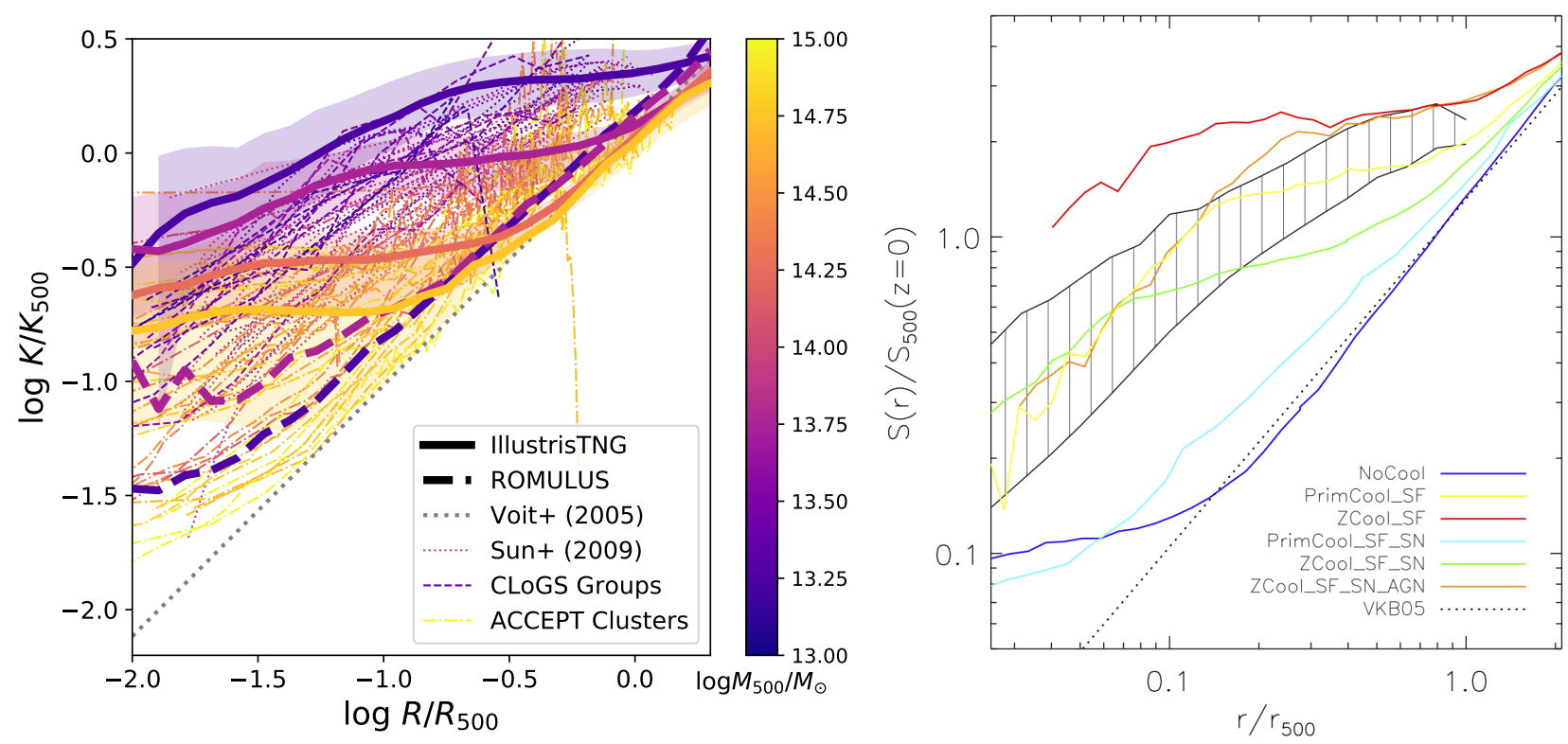

Figure 6. (Left) Normalized mass-weighted 3D entropy profiles at $z \sim 0$ for groups and clusters with $M_{500}=10^{13}-10^{15} \mathrm{M}_{\odot}$ in TNG100 and TNG300 (combined, thick solid lines) and ROMULUS (thick dashed lines). We show median-stacked profiles of halos in 0.5 dex bins, with 264 (7), 110 (5), 91, and 15 halos per bin for TNG (ROMULUS) in order of increasing mass, color-coded by mass from low-mass groups (purple) to massive clusters (yellow). For TNG, we also indicate their $1 \sigma$ scatter with shaded bands. These predictions are compared to individual halos from three observed samples, all colored analogously by halo mass: low-mass CLoGS groups (dashed lines [8]), massive groups (dotted lines[2]), and a random $20 \%$ subset of the ACCEPT clusters (dash-dotted lines [9]). The gray dotted line indicates the $K \propto r^{1.1}$ scaling found in non-radiative simulations [96]. In agreement with observations, TNG (but not ROMULUS) predicts higher normalized entropy at lower mass, but with higher-entropy cores than observed. In contrast, ROMULUS groups have steeper and lower entropy profiles that are more typical of observed massive clusters. (Right) Normalized mass-weighted 3D entropy profiles of $M_{500}=10^{13.25}-10^{14.25} \mathrm{M}_{\odot}$ groups predicted by OWLS simulations with different subgrid models [116], reproduced with permission from McCarthy et al. [158] (note that their symbol for astrophysical entropy is $S$, rather than K). Simulations include a run without any cooling or feedback (blue); without feedback but with star formation and cooling without (yellow) or with (red) the contribution from metal lines; with the addition of SNe feedback (cyan and green, respectively); and with AGN feedback added in addition to metal-line cooling, star formation, and SNe feedback (brown). The black-hatched band indicates the $1 \sigma$ scatter of the Sun et al. [2] observations, the black dotted line a $r^{1.1}$ power law. While AGN feedback has a clear effect on the IGrM, the entropy profiles are also sensitively affected by cooling, star formation, and SNe feedback.

Interestingly, such a trend is not seen in ROMULUS, although the small number of groups and absence of massive clusters prevents strong conclusions here. If anything, the lower-mass groups (blue dashed line) have entropy profiles that are even closer to observed CC clusters, plausibly also because of a late-time merger in the more massive ROMULUSC group [49]. As discussed further below, it is plausible that this CC-like behaviour of ROMULUS groups is due to the absence of metal-line cooling-which may limit the removal of low-entropy gas from its IGrM-as well as the highly collimated nature of their AGN-driven outflows.

There are additional reasons why the ubiquitous conversion from CC to NCC halos in most simulations is problematic. McCarthy et al. [216] showed that the cost, in energy, is much greater than the typical jet power of an AGN outburst [217], with most of the energy being expended to lift the gas rather than raise its entropy. This suggests that the AGN outbursts in the simulations are likely injecting much more energy than observed radio AGNs in galaxy clusters. Observations also show little evidence of AGN feedback affecting CC-to-NCC transformations. The entropy profiles of NCC as well as CC CLoGS groups (with and without observed jet activity) are all, to first order, similar in shape and normalization $[2,8]$. Moreover, none of the clusters showing evidence of having experienced an extreme AGN feedback event in the recent past-e.g., MS 0735.6+7421 [218] and Hydra 
A $[217,219]$ — have core entropies as high as the median value in the simulations. We therefore speculate that the action of the AGN feedback models in SIMBA, TNG, and (C-)EAGLE may be too aggressive in the low- $z$ Universe (see also the discussion in Barnes et al. [43]). Although we are comparing individual observed profiles to medians from simulations, our examination of simulated individual profiles (not shown) indicates that the median profiles are fairly representative, so that this is unlikely a source of significant bias.

Given the mismatched entropy profiles in contemporary simulations, it is helpful to refer to McCarthy et al. [158], who demonstrated using multiple OWLS simulations that adding cooling to non-radiative simulations can alter the entropy slopes as much as feedback (Figure 6, right panel). While non-radiative simulations fall on the baseline $K \propto R^{-1.1}$ relationship, adding only cooling without feedback "cools out" low-entropy gas, raising the entropy of the remaining IGrM. Metal-line cooling, which is comparatively more important for groups than clusters, raises entropy even more. Adding $\mathrm{SNe}$ feedback without metal-line cooling actually lowers the entropy nearly to the $R^{1.1}$ line, which may explain the ROMULUS entropy slopes that have weak AGN feedback that is more similar to SNe feedback and no metal-line cooling. While McCarthy et al. [158] confirm that adding AGN feedback indeed increase the entropy (cf. orange vs. green lines), the difference is smaller than changing the cooling physics. Furthermore, they showed that the AGNinduced entropy increase is, for the largest part, also an indirect effect: in their simulation, it is not (primarily) caused by heating gas that remains in the IGrM, but by the selective ejection of low-entropy gas from the less tightly bound $z \approx 2-4$ progenitor halos.

We end our discussion of IGrM properties by pointing out that even radial profiles convey only a simplified view of the processes shaping the gaseous halos of groups. This is exemplified particularly clearly by high-resolution simulations such as ROMULUSC, for which we show 2D maps of the gas temperature and density near the center of its most massive group at $z=0.53$ in Figure 7 (adapted from Figure 10 of Tremmel et al. [49]). A biconical jet is clearly seen in the temperature map, which emerges naturally by collimation of their (intrinsically isotropic) AGN feedback by a central gas disc. This jet evacuates bubbles in the vicinity of the group center-not unlike X-ray cavities observed in real clusters (e.g., Hlavacek-Larrondo et al. [220]) — but does not strongly affect gas away from its axis, thus preserving a steep $R^{1.1}$ entropy slope down to $0.04 R_{500}$ in a spherically averaged sense (Figure 6).

\section{$1000 \mathrm{~km} / \mathrm{s}$}
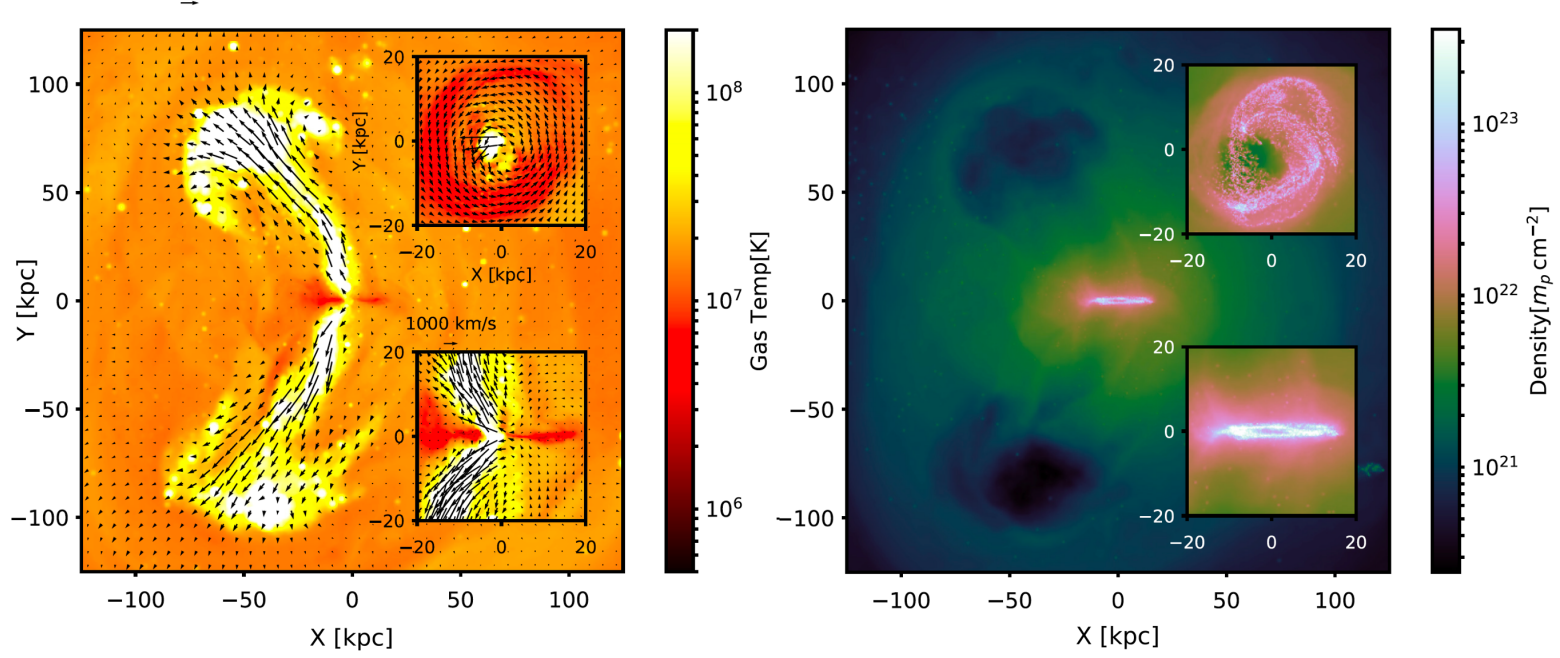

Figure 7. Wind-blown bubbles driven by AGN feedback from the central galaxy in the ROMULUSC simulation at $z=0.53$, adapted with permission from Tremmel et al. [49]. This $M_{500}=10^{13.6} \mathrm{M}_{\odot}$ group produces a cool core that is elusive in other simulations, although the central gas densities are high compared to observations. This AGN feedback is a thermal dump model without explicit collimation, and is the most obvious example of bubble-driven feedback at the group scale found in the cosmological simulations we explore. 


\subsection{Brightest Groups Galaxies}

For more than two decades, attempts to model the formation and evolution of galaxy groups and to identify the role of different physical processes that shape these systems have focused primarily on the properties of the hot IGrM/ICM ( e.g., [202,213,216,221-223], and references therein). Even with the advent of cosmological simulations, this tendency has largely continued $[7,37,39,44,109,132,204,215,224]$. However, observational studies find that a number of BGG properties (e.g., stellar mass, size, morphology, the nature of the surface brightness and stellar velocity dispersion profiles, whether the BGG is a fast or slow rotator, etc.) are also correlated with the properties of their host system [155,225-231]. Such correlations are not entirely unexpected given that BGGs are typically found at the bottom of their host halo's gravitational potential well. As such, the BGGs are thought to have experienced numerous mergers and close tidal encounters with other group galaxies over cosmic time. Simulations suggest that such interactions result not only in the growth of the BGGs' total stellar mass but also, induce structural and kinematic transformations [232-237]. Mergers can also potentially transport in cool gas and fuel in-situ star formation in disc-like structures. Additionally, as explicitly demonstrated by Lewis et al. [95] using the first hydrodynamic cosmological simulation of a rich galaxy group/poor cluster that allowed for radiative cooling, any gas cooling out of the IGrM/ICM flows towards the group center and ends up in the central galaxy. Consequently, the study of the BGGs, their properties, and the existence of any correlations between the latter and the properties of the host groups offer a complementary window onto the physical processes underlying the evolution of galaxy groups. Just as importantly, in the context of the present review, detailed comparisons of the observed BGG properties and the characteristics of those in numerical simulations offers an equally powerful opportunity for testing the efficacy of latest generation of cosmological simulations. In recent years, a handful of papers have assessed the characteristics of BGGs forming in hydrodynamic simulations (e.g., [7,39,43,48-50,238]) although this was done as part of a broad survey of the general properties of simulated galaxies. There are however notable recent exceptions, like Davison et al. [239], Henden et al. [240], Jackson et al. [241], Pillepich et al. [67], Katsianis et al. [242], Remus et al. [243], Tacchella et al. [244] and Jung et al. [73], that have treated BGGs as a distinct class, investigating both the structural and the kinematic properties of the simulated BGGs and comparing these to observations.

\subsubsection{Central Galaxy Stellar Masses}

The first BGG (and BCG) property we consider is their stellar mass-halo mass (SMHM) relationship. This is shown in Figure 8, where we plot $M_{\star} / M_{200}$ against $M_{200} . M_{\star}$ is the mass of the stars associated with a BGG/BCG. Stellar observations show that once the light from individual resolved galaxies other than the central BGG/BCG is excluded, the resulting surface brightness can be separated into two components: that due to light from a localized concentration of stars usually identified with the BGG/BCG proper and that due to light from a diffuse, often extended, component: the intragroup/intracluster light (IGrL/ICL) (e.g., see [245]). The $M_{\star}$ we quote here is the sum of the mass in the two components. The SMHM offers a window onto not just the efficiency with which the gas in the central galaxies is turned into stars but also the tug-of-war between heating and cooling in the group cores as well as the role of mergers in the build-up of the central plus IGrL stellar mass.

For the purposes of clarity, we plot the simulation results across the three panels in Figure 8 but for reference and comparison, we show the same set of nine representative observationally determined SMHMs ${ }^{12}$ in all three panel. All of the observed results are based on the projected stellar light distribution and correspond to the central+IGrL stellar mass within a cylinder aligned along the line of sight with some aperture of radius $R$ (i.e., $M_{\star, R, 2 D}$ ). This mass estimate explicitly excludes the contribution from resolved satellite galaxies. The dashed, dotted and solid curves are results from Girelli et al. [247], Yang et al. [248,249], Moster et al. [250], van Uitert et al. [251], Erfanianfar et al. [252]. There are two sets of Kravtsov et al. [207] datapoints: the filled stars are based on $M_{\star, 50,2 D}$ and the 
open stars are based on "total" aperture masses, derived by extrapolating and integrating the ICL profiles to large radii in the sky $\left(M_{\star, \text { tot, } 2 D}\right)$. The blue crosses with error bars are total aperture masses from Loubser et al. [231] and Kolokythas et al. [253]: points with $\log \left(M_{200} / \mathrm{M}_{\odot}\right)<14.0$ are for BGGs from the high richness subset of CLoGS groups [8] while the BCG results are from the Multi Epoch Nearby Cluster Survey and the Canadian Cluster Comparison Project (MENeaCS and CCCP, respectively [254-259]). There is a considerable spread in the published observationally derived SMHM results. This spread is due to a variety of factors, including (i) the groups and clusters are sourced from deep targeted observations as well as large surveys (e.g., SDSS, COSMOS and GAMA) of varying depth; (ii) the use of apertures of different sizes; (iii) the use of different strategies to estimate the background around large massive galaxies in crowded environments [207], as well as to model and extrapolate their observed light profiles of interest; (iv) the use of different estimates s of $M_{\star} / L$ ratio to convert measured light distribution into stellar mass; and (v) the use of different approaches for estimating the halo mass, ranging from the use of X-ray observations under the assumption of hydrostatic equilibrium and abundance matching, to weak gravitational lensing estimates. The observational results shown in Figure 8, taken together, show how $M_{\star} / M_{200}$ scales with $M_{200}$. We interpret the spread in the SMHM determinations as a measure of the uncertainty when comparing to the simulation results.

As for the simulations, we first consider the results from the ROMULUS simulations (i.e., ROMULUS25 [48], ROMULUSC [49,72,73], ROMULUSG1 and ROMULUSG2 [73]). These are plotted in the first (top left) panel. Following Liang et al. [37], Robson and Davé [215], and Jung et al. [73], we identify systems with $\log \left(M_{200} / \mathrm{M}_{\odot}\right) \geq 12.5$ as groups/clusters; these are shown as filled yellow circles. The lower mass systems are plotted as open yellow circles. The $M_{\star}$ for ROMULUS systems is the projected stellar mass within an $R=50 \mathrm{pkpc}$ aperture (i.e., $M_{\star, 50,2 D}$ ). The $M_{\star} / M_{200}$ for the ROMULUS groups is in very good agreement with the observations; however, the overall trend suggests a drop with increasing $M_{200}$ that is not as steep as the observed trend.

In the same panel, we also show the results for TNG100 (cyan line/band; [67]) and FABLE (magenta line/band; [240]) simulations. The available TNG stellar masses are $M_{\star, 30,3 D}$, that is, stellar mass within a sphere of radius $R=30 \mathrm{pkpc}$. We have corrected these masses to stellar mass within a 50 pkpc sphere using mass profiles from Pillepich et al. [67]. We suggest that it is preferable to compare this corrected stellar mass to the observed aperture masses. The FABLE results are $M_{\star, 50,2 D}$, like ROMULUS. The thick solid line is the median SMHM relationship and the shaded region encompasses $95 \%$ of the systems. Both TNG and FABLE results are in very good agreement with the observations on the group and low-mass cluster scales (i.e., $\log \left(M_{200} / \mathrm{M}_{\odot}\right)<14.0$ for TNG and $\log \left(M_{200} / \mathrm{M}_{\odot}\right)<14.3$ for FABLE). However, neither the TNG nor the FABLE median curve decreases as steeply as the observed relationship on the cluster scales. We also note that in the case of TNG, the median curve may become shallower still if one were to use a cylindrical volume.

In the second (top right) panel, we show the results from SIMBA (red line/band; [50]) and The Three Hundred project (brown line/band; [45]). Both results are $M_{\star, 50,2 D}$. Considering the SIMBA results first, we find that on the group scale, that is, $\log \left(M_{200}\right)=$ $[12.5,13.8]$, the distribution of $M_{\star} / M_{200}$ points for individual SIMBA BGGs (not explicitly shown) is consistent with the observations. The median curve, however does not have as steep a slope as the SMBHs based on observations. Consequently, the SIMBA central galaxies on the cluster scale (BCGs) have larger stellar mass than their observed counterparts and the discrepancy grows with increasing halo mass. Overall, SIMBA is in modest agreement with the observations when compared with observational results across the entire span, from low mass groups to massive clusters. The Three Hundred SMHM does not extend to low mass groups but over the mass range covered $\left(\log \left(M_{200}\right)=[13,15]\right)$, the relationship is in excellent agreement with the observations. 

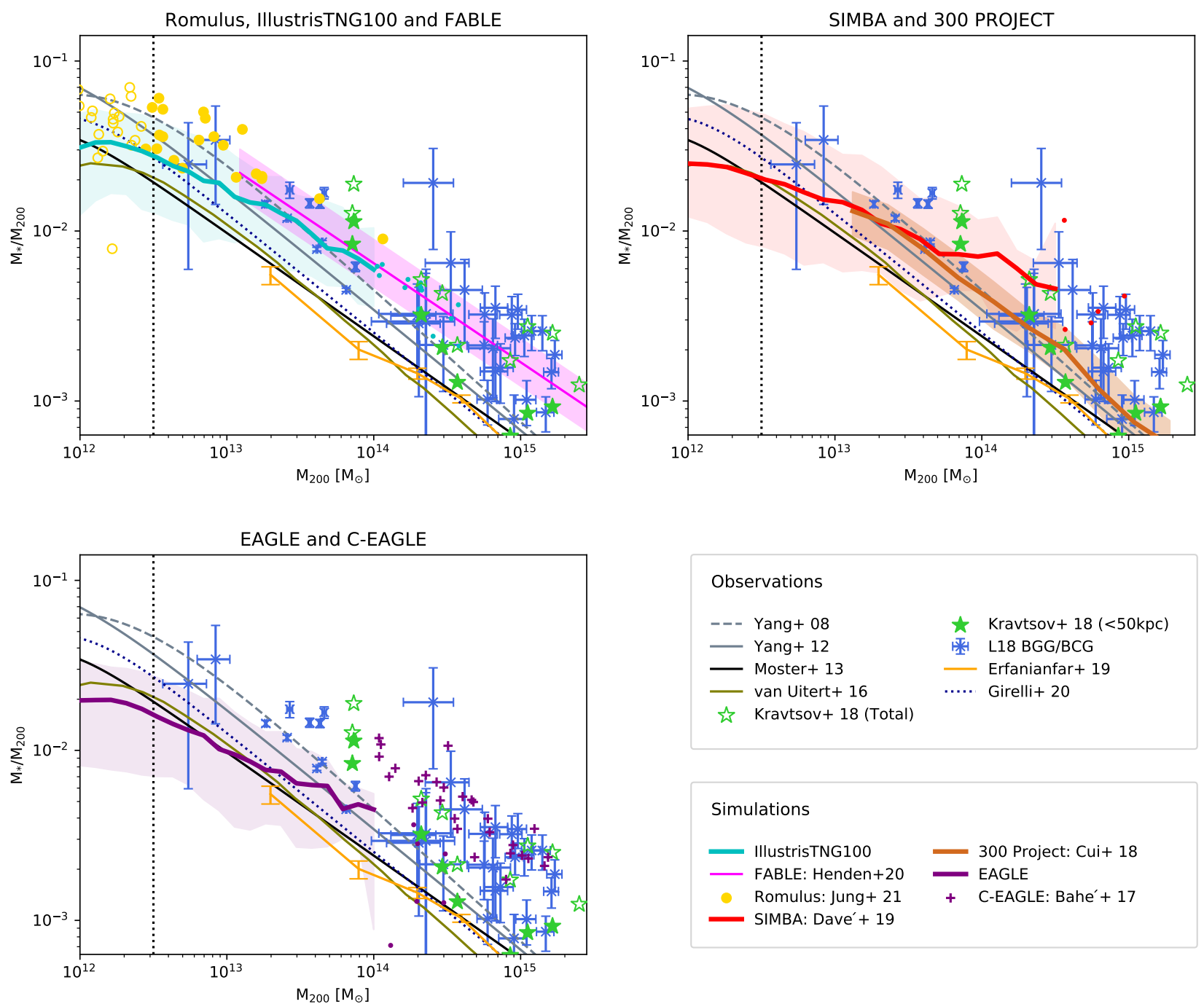

Figure 8. Figure adapted from Jung et al. [73]: The $z=0$ stellar mass-halo mass relationship $\left(M_{\star} / M_{200}\right)$ versus $M_{200}$ for central galaxies in observed and simulated galaxy groups and clusters. The top left panel shows results for TNG100 (turquoise line/band and dots; [67]), FABLE (magenta line/band; [240]), and ROMULUS suite of simulations (open and filled yellow points; $[49,72,73])$. The thick solid lines are the median SMHM; the shaded region spans $95 \%$ of the systems. The top right panel shows results for SIMBA (red line/band and dots; [50]) and The Three Hundred project (brown line/band; [45]). The bottom left panel shows results for the EAGLE (purple line/band and dots; [39]) and C-EAGLE (purple crosses; [42]). We identify systems with $\log \left(M_{200} / \mathrm{M}_{\odot}\right) \geq 12.5$ (to the right of the dotted vertical line) as groups/clusters. The ROMULUS, FABLE, SIMBA, C-EAGLE and The Three Hundred project stellar masses are $M_{\star, 50,2 D}$; EAGLE and TNG stellar masses are $M_{\star, 30,3 D}$ corrected to $M_{\star, 50,3 D}$. For comparison, we also plot the same nine observationally determined SMHMs in all three panels. These are described in the text. There is considerable spread between the nine relationships but jointly, they show how $M_{\star} / M_{200}$ scales with $M_{200}$. We interpret the spread as a measure of the uncertainty.

The third (bottom left) panel shows the results from C-EAGLE (purple crosses Bahé et al. [42]) and the EAGLE reference run (purple line/band; [39]). The C-EAGLE stellar masses are $M_{\star, 50,2 D}$. The results for the lowest mass C-EAGLE systems are consistent with the observations but in massive clusters, as Bahé et al. [42] notes, the C-EAGLE stellar masses exceed the $M_{\star, 50,2 D}$ (filled stars) from Kravtsov et al. [207] by up to 0.6 dex. As for EAGLE, the reported $M_{\star}$ is $M_{\star, 30,3 D}$; we used a mean stellar mass-dependent correction factor, derived from plots in Schaye et al. [39] and McCarthy et al. [41], to map $M_{\star, 30,3 D}$ to $M_{\star, 50,3 D}$. The correction factor is negligible for galaxies with $\log \left(M_{\star, 30,3 D} / \mathrm{M}_{\odot}\right)<10.7$ [39]; on the other hand, the stellar mass in the most massive BGG/BCGs approximately doubles. Over the mass range $\log \left(M_{200}\right)=[13,14]$, the distribution of individual $M_{\star} / M_{200}$ EAGLE points (not 
explicitly shown) is consistent with the observed SMHM results; however, like the SIMBA results, the EAGLE median curve is shallower than the observed trend. Consequently, for $\log \left(M_{200} / \mathrm{M}_{\odot}\right)<13$, more than half of EAGLE BGGs fall below Moster et al. [250] line, which is the lowest of the observationally-derived SMHM curves. Overall, the EAGLE SMHM is in modest agreement with the observations across the entire span, from low mass groups to massive clusters. Finally, we point out that the EAGLE median curve in Figure 8 is flatter than that in Schaye et al. [39] because of the mass-dependent correction, and we expect that the corresponding results based on $R=50 \mathrm{pkpc}$ aperture mass will be shallower still since our correction maps to a sphere, not a cylinder.

\subsubsection{Central Galaxy Star Formation Rates}

Figure 9 shows the second trend that we consider here: the star formation rate-stellar mass relationship (hereafter, SFR $-M_{\star}$ ). The SFR is based on star formation within a $R=30$ pkpc sphere centered on the BCG (very little star formation is observed beyond $30 \mathrm{pkpc}$ ) while the $M_{*}$ is the same as that in Figure 8. We first consider the four sets of observational data points that appear in all three panels: (i) results based on combined data from XMM-LSS, COSMOS, and AEGIS surveys for BGGs and BCGs hosted by X-ray bright galaxy groups and clusters (green pluses; [229]); (ii) results for BCGs in Mittal et al. [260] sample of CC clusters (grey crosses); (iii) results for the high richness subset of the CLoGS sample (blue crosses; $[8,231,253])$, which consists of groups containing at least 4 optically bright $\left(\log \left(L_{\mathrm{B}} / L_{\odot}\right) \geq 10.2\right)$ galaxies, of which the central galaxy is an ETG; and (iv) results for BCGs from the COSMOS survey (magenta squares; [261]).

The Gozaliasl et al. [229], Loubser et al. [231], Cooke et al. [261] and Mittal et al. [260] data collectively illustrate the approximate dichotomy in the population of BGGs and BCGs with respect to their star formation properties. On the cluster scale, this phenomenon has been widely discussed (e.g., [255,263], and references there in) and linked to the CC/NCC dichomotomy in the X-ray properties, including the X-ray luminosity at fixed halo mass, the core entropy value and the core hot gas cooling time as highlighted by Cavagnolo et al. [9], McCarthy et al. [216,264] and others. Essentially, not all BCGs are "red and dead"; there exists a population of star-forming BCGs that reside in strong cool core clusters [255]. These BCGs' SFRs are such that they are distributed within approximately \pm 0.75 dex of the extension of the observed $z<0.5$ star forming main sequence (SFMS) of Whitaker et al. [262]. The star-forming BCGs comprise $\sim 25 \%-30 \%$ of all BCGs; this is also the fraction of strong CC clusters [256,265]. Gozaliasl et al. [229] find that this dichotomy is also present on the group scale and the fraction of star-forming BCGs is comparable to that of star-forming BCGs; that is, $20 \%-25 \%$. The majority of the observed central galaxies in Figure 9 have SFRs less than 0.75 dex below the SFMS. We refer to these as "quenched" BGGs/BCGs.

As for the simulations, we restrict ourselves to groups and clusters whose BGG/BCG satisfy $\log \left(M_{\star} / \mathrm{M}_{\odot}\right) \lesssim 12.0$; there are too few systems with higher stellar masses. We classify the simulation BGGs/BCGs in the same way as the observed systems: We designate all simulation BGGs/BCGs with SFRs less than 0.75 dex below the observed $z<0.5$ SFMS [262] as "quenched" systems, and the rest of the galaxies as star-forming. The numbers at the bottom of the panels specify the fraction of quenched galaxies in 0.5 dex $M_{*}$ bins for TNG100, SIMBA, and EAGLE simulations. Many of the quenched galaxies have low / unresolved SFRs. In order to display these galaxies in the panels, we assign them an $\mathrm{SFR}$ of $\log \left(\mathrm{SFR} / \mathrm{M}_{\odot} \mathrm{yr}^{-1}\right)=-4$. To the extent that it is of interest, we find that the number of galaxies with very low/unresolved SFRs relative to the total number varies considerably from simulation to simulation. However, we do not differentiate between quenched galaxies with low/unresolved SFRs and those with low but measurable SFRs, and there is there is no observational basis for doing so. Measuring low SFRs using observationally accessible diagnostics is extremely challenging [266-271], and strictly speaking, very low published SFRs ought to be treated as upper limits. 

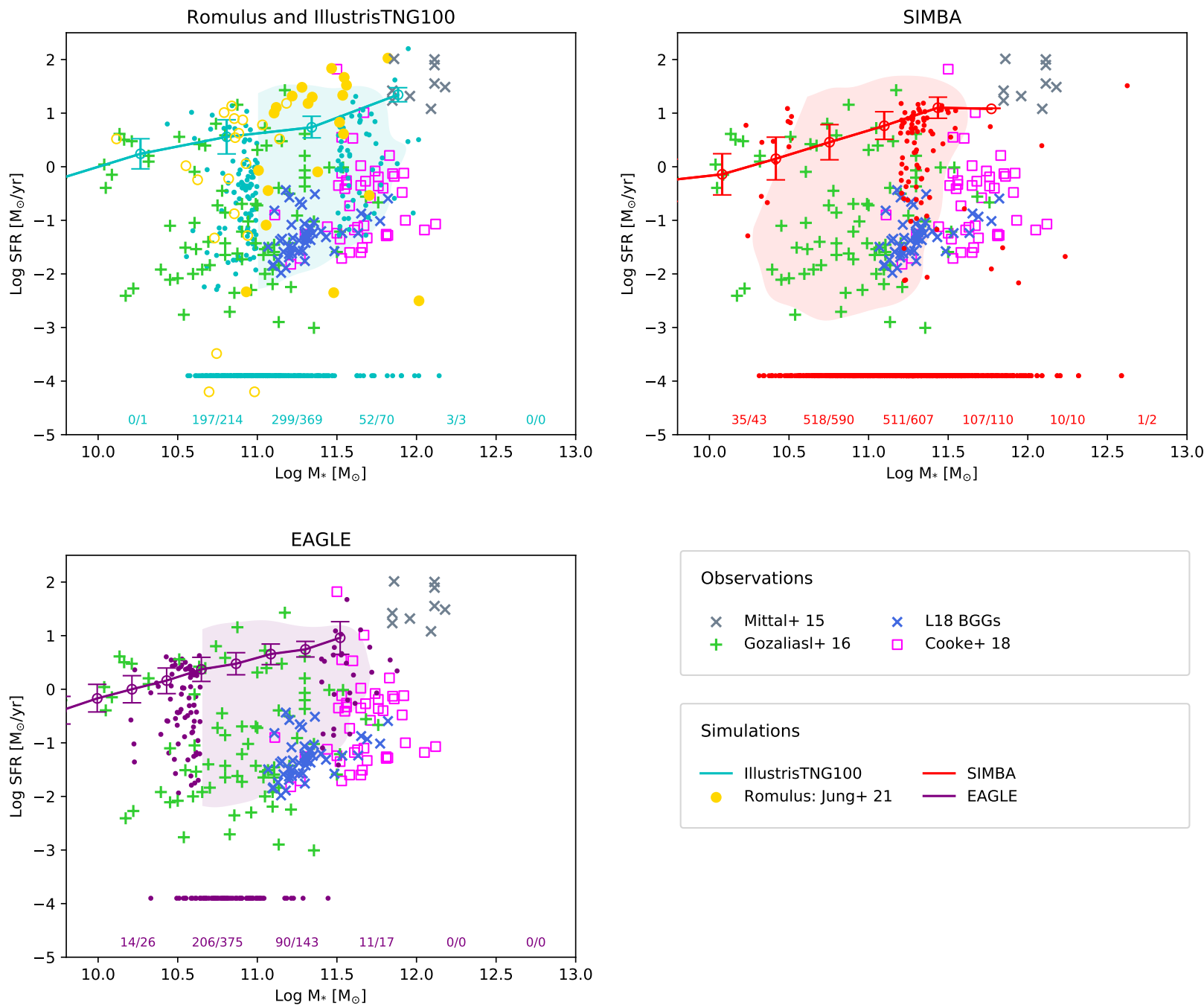

Figure 9. Figure adapted from Jung et al. [73]: Comparison of the $z=0$ star formation rate (SFR)- $M_{\star}$ relationship for observed and simulated BGGs and BCGs. Arranged as Figure 8, each panel plots the same four sets of observational results (see text for details). The top left panel shows the results for ROMULUS (yellow filled and open circles; filled circles represent systems we identify as groups/clusters) and TNG100 simulations (cyan); the top right panel shows the results for SIMBA (red); and the bottom left panel shows the results for EAGLE (purple). The simulation SFRs are extracted from a sphere of radius 30 pkpc encompassing the central BGG/BCG while the stellar masses as the same as in Figure 8 . The line connecting the open circles with error bars shows the star forming main sequence (SFMS) for TNG100, SIMBA, and EAGLE from Davé et al. [154]. The shaded regions, and the handful of individual points to the left and right, show where the simulation BGGs/BCGs with measurable SFR lie on this plot. BGGs/BCGs with unresolved/too low SFRs are assigned $\log \left(\mathrm{SFR} / \mathrm{M}_{\odot} \mathrm{yr}^{-1}\right)=-4$ and plotted accordingly. We identify all simulation BGGs/BCGs with SFRs $\gtrsim 0.75$ dex below the observed $z<0.5$ SFMS of Whitaker et al. [262] as "quenched". For TNG100, SIMBA, and EAGLE, we specify the fraction of quenched galaxies in $0.5 \operatorname{dex} M_{*}$ bins at the bottom of the panels. We do not differentiate between quenched systems with low but measurable SFRs and those with very low/unresolved SFRs. Measuring very low SFRs using observationally accessible diagnostics is extremely challenging; strictly speaking, the corresponding published SFRs ought to be treated as upper limits.

Considering the ROMULUS results (yellow points) in the top left panel first, we find that this model gives rise to both star-forming and quenched BGGs; however, the corresponding fractions are inverse of the observed fractions: $~ 60 \%$ of the ROMULUS BGGs are star-forming and $\sim 40 \%$ are quenched. The top left panel also shows the entire TNG100 population of BGG/BCGs. Only $17 \%$ of these are star-forming. This is lower than the observed fraction. The SIMBA results in the top right panel are similar to TNG100 and also on the low side: only about $14 \%$ of all the SIMBA BGGs/BCGs are star-forming. 
The fraction of EAGLE BGGs (lower left panel) that are star-forming is $\sim 40 \%$, which is higher than the observed fraction though not as high as the ROMULUS fraction. We will return to these results in Section 4.2.4.

\subsubsection{Central Galaxy Morphologies}

The final trend we consider is the stellar mass-morphology relationship for the central galaxies. Qualitatively, the observed morphologies of central galaxies span the full continuum, from disk dominated to pure spheroids. Weinmann et al. [228] found that approximately $50 \%$ of the BGGs in low-mass SDSS groups are late-type galaxies (see also [272]); this fraction drops to $\sim 10 \%$ in rich groups / poor clusters. Conversely, the fraction of earlytype central galaxies rises from $\sim 30 \%$ in low-mass groups to $70 \%$ in high-mass systems. The morphological mix of the ROMULUS BGGs, which are from mainly low-mass groups, is consistent with Weinmann et al. [228] results in that nearly a half are disky and star forming [73]. Similarly, a visual inspection of the images of BCGs from the 20 most massive TNG100 systems with halos masses between $9 \times 10^{13} \mathrm{M}_{\odot}$ and $4 \times 10^{14} \mathrm{M}_{\odot}$ ( [67]) also suggests that their morphological types are also compatible with Weinmann et al. [228] results for low-mass clusters: A significant fraction are ellipsoidal or S0-like and appear to be red.

More quantitatively, a galaxy's morphology is commonly characterized by the fraction of its total stellar mass that is in the spheroidal component; that is, its spheroidal-to-total ratio $(S / T)$. This ratio has been computed using photometric observations, by decomposing the 2D projected light profile into disc and spheroidal components, but it can also be derived using stellar kinematics to identify the spheroidal component. The two approaches do not also give similar results. Simulation studies have shown that photometrically derived S/Ts tend to be significantly lower than the kinematic S/Ts [273], with Bottrell et al. [274] finding that the former leads to a higher likelihood of a galaxy being classified as "disky even when stellar kinematics show no ordered rotation". In effect, the Scannapieco et al. [273] and Bottrell et al. [274] studies show that it is not sufficient to only consider the BGG/BCG properties derived from photometric data. There is much to be learnt from examining the galaxies' kinematic properties.

In Figure 10, we show the kinematic $S / T$ ratios for the central galaxies from ROMULUS [73], TNG100 [244] and EAGLE [238], as a function of galaxy stellar mass. We use comparable apertures as Tacchella et al. [244] to compute ROMULUS $S$ / $T$ ratios: For BGGs with $\log \left(M_{*} / \mathrm{M}_{\odot}\right) \leq 10.9, S / T$ s are computed using star particles within spheres of radius $R=15-20 \mathrm{pkpc}$; for more massive BGGs, we use spheres of radius $R=25-30 \mathrm{pkpc}$. We also use the same criterion to define the spheroidal component as Tacchella et al. [244]: the mass of the spheroid is the sum of the mass of stellar particles with $\epsilon_{\mathrm{J}}<0.7$ and the $15 \%$ of the stellar particles with $\epsilon_{\mathrm{J}}>0.7$, where $\epsilon_{\mathrm{J}}=J_{\mathrm{z}} / J_{\text {circ }}(E), J_{\mathrm{z}}$ is a z-component of the specific angular momentum of a stellar particle, $\mathrm{z}$ is the net spin axis of a galaxy, and $J_{\text {circ }}(E)$ is a specific angular momentum of the stellar particle on a circular orbit with the same orbital energy.

The EAGLE criterion for determining $S / T$ ratios differs slightly but we have confirmed that the two criteria give comparable results. Figure 10 also shows the $S / T$ for central galaxies in the GAMA groups sample derived using photometric data (orange points; [13]), as well as the kinematic $S$ / $T$ for BGG/BCGs from the CALIFA survey (red points; [275]). We use the same prescription as Tacchella et al. [244] to compute the CALIFA $S / T$ s. The simulation results should be comparable to the CALIFA results.

Overall, there is a broad agreement between the three simulation results and for $M_{*} \gtrsim 10^{11} \mathrm{M}_{\odot}$, all three simulation results are consistent with the CALIFA results. In these galaxies, the spheroidal component dominates: $S / T$ rises from approximate 0.7 to 0.9 with stellar mass. Towards lower $M_{*}$, however, the median TNG100 and EAGLE curves diverge from the CALIFA results. At present, it is unclear how much weight this divergence ought to be given. Firstly, there is a considerable spread in the simulation results and the CALIFA results are well within the shaded region; secondly, the CALIFA sample is known to be 
increasingly incomplete towards at lower masses. It is possible that the trend in the CALIFA results reflects this incompleteness.

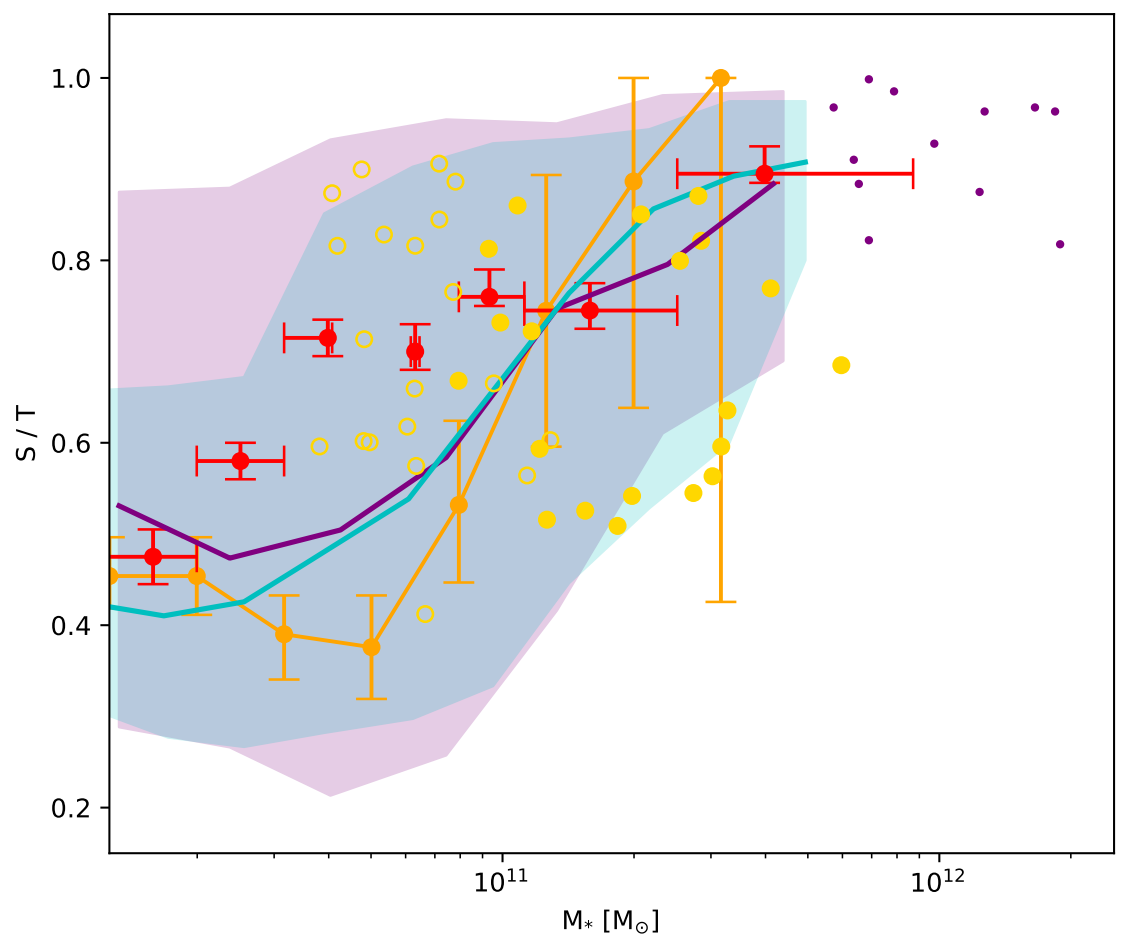

Simulations

EAGLE: Clauwens+ 18

IllustrisTNG100: Tacchella+ 19

Romulus: Jung +21

Observations

GAMA: Moffett+ 16

- CALIFA: Zhu+ 18

Figure 10. Figure adapted from Jung et al. [73]: The $z=0$ spheroidal-to-total $(S / T)$ for central galaxies in simulated galaxy groups and clusters from three simulations (ROMULUS, TNG100, and EAGLE), juxtaposed against results from two observational studies. The yellow filled and open circles show the ROMULUS kinematically derived results. (As in previous two figures, filled circles correspond to systems we identify as groups and clusters.) The cyan and purple curves show the median kinematically derived S/T results for TNG100 [244] and EAGLE [238], respectively. The purple shaded region indicates the $10-90 \%$ range while the cyan shaded region spans the $16 \%-84 \%$ range. The ROMULUS and TNG100 $S / T$ are computed using the same criterion while the EAGLE results are based on a slightly different criterion. The two criteria, however, give very similar results. Of the two observational results shown, the CALIFA data are kinematically derived [275] while the GAMA results are based on photometry [13]. The two approaches are not equivalent. We discuss this further in the text. The simulation results should, in the first instance, be compared to the CALIFA results.

Finally, we note that $S / T$ is not the only kinematic measure that is correlated with either the central galaxies' stellar mass or the host systems' properties. Other measures less commonly discussed in the galaxy formation literature include (i) the shape of the velocity dispersion profiles and whether they rise or fall with radius; (ii) the "anisotropy parameter" $V_{\text {rot }} / \sigma_{0}$, which characterizes the global dynamical importance of rotation and random motions of stars in a galaxy; and (iii) whether the galaxy is a fast or slow rotator. Jung et al. [73] investigates some of these measures for ROMULUS galaxies and compare them to observations and we refer interested readers to that paper for further details and discussion. 


\subsubsection{The Link between the BGG and IGrM}

The properties reported in Figures 8-10 are the observable byproducts of a myriad of physical processes that the BGGs and BCGs are subject to over the course of cosmic time. These properties are also sensitive to the details of the subgrid prescriptions used to model those processes that cannot be directly resolved in the simulations. All three of the properties discussed in this subsection are related to each other and more importantly, they are all correlated with the the current or the recent state of the IGrM/ICM in the group and cluster cores. Here, we briefly elaborate on this relationship, focusing on the SFR- $M_{\star}$ results.

As highlighted throughout this review, among the most important present-day challenges in simulating galaxy groups and clusters is (i) preventing strong radiative cooling flows from forming in the IGrM/ICM and (ii) ensuring that the resultant population of groups and clusters span the spectrum from CC to NCC systems. Nearly two decades ago, Babul et al. [213], Valageas and Silk [276], Nath and Roychowdhury [277] advocated for AGN feedback as the key mechanism for addressing these challenges. The current generation of simulation models of galaxy formation/evolution (see Table 2) all allow for SMBH seeding and growth as well as AGN feedback although the manner in which these are implemented vary from one simulation to another.

To examine how well the current models fare, we first consider the ROMULUS simulations. As noted previously, the fact that nearly $60 \%$ of the ROMULUS BGGs are star-forming (Figure 9) means that while AGN feedback in ROMULUS may temper cooling, it does not entirely halt it and consequently, a majority of the systems sustain some degree of cooling flow in their cores. A closer examination of the ROMULUS entropy profiles, such as those shown in Figure 6, offer some insights. For the most part, ROMULUS groups behave like CC clusters, not CC groups. Their entropy profiles are $\sim 2-3 \times$ lower than the Sun et al. [2] profiles at large radii, and the profiles themselves are steeper $\left(R^{1.1}\right.$ versus $\left.R^{0.7}\right)$. We suspect this is the result of a combination of AGN feedback being too weak and metal-line cooling being absent. ROMULUS simulations do not produce stable NCC systems although once in a while, they do produce weak NCC systems [72] and during this phase, the BGG is quenched [73]. This phase typically arises when the BGG suffers a sizable merger that elevates the core entropy; however, the phase is temporary, lasting $\lesssim 2$ Gyrs.

Turning to the TNG and SIMBA simulations, we find that $78 \%$ and $91 \%$ of the BCGs, respectively, with $\log \left(M_{*} / \mathrm{M}_{\odot}\right) \geq 11.3$ are quenched. Such high fractions are not surprising given that the vast majority of the $z=0$ TNG and SIMBA clusters are NCC systems with high entropy cores (see, for example, the orange and yellow curves in Figure 6). Neither TNG nor SIMBA produce clusters with typical CC cluster entropy profiles, and in both cases, the main reason appears to be that feedback is both too efficient, resulting in larger and higher entropy central cores than observed (c.f. [214,215]).

The main takeaway, therefore, is that while all of the simulation models appear to find reasonable agreement with a subset of the available observations of galaxy groups and clusters, none of them correctly describe galaxy groups and clusters in a comprehensive manner. It is very likely that the problems with the entropy profiles and quenched/starforming BGGs/BCGs are due to either a problem with the physical models that inform the subgrid modeling of the various aspects of SMBH formation and evolution, and/or due to the implementation these models in the simulations. All simulation models have ad hoc features embedded within their subgrid prescriptions and when the models run into difficulties, quite reasonably a common response is to attempt to patch one or more of the subgrid schemes in the hope that that fixes the problem without causing any breakage elsewhere. In the case of AGN feedback, we assert that minimal patches have generally not led to a realistic population of simulated galaxy groups and clusters. This should be seen as a clear invitation to revisit the treatment of SMBHs and AGNs in the simulations. We offer some thought on the subject in Section 5.3. 


\subsection{The Multiphase IGrM}

Observations of the IGrM are dominated by the X-ray emission from hot, dense gas surrounding the BGG. Since X-ray emission scales with the square of gas density, diffuse warm and cool phases of the IGrM are prohibitively difficult to observe directly in emission. While several groups have pioneered efforts to characterize the warm and cool IGrM/ICM with UV absorption specifically targeting group environments $[27,278,279]$, the Virgo [280,281] and Coma [282] clusters, other clusters [26,283], cluster outskirts [284,285], as well as low-mass group halos hosting ETGs [286], such studies are limited by the available number of background sources with existing instrument sensitivities. For this reason, simulations of groups offer a unique insight into the multiphase structure of the IGrM and the physical origins of its different components. Because warm and cool gas is expected to form structures that are significantly smaller than the volume-filling hot phase, simulations that aim to accurately model the multiphase IGrM require extremely high resolution. Recent advancements in the resolution of group-scale simulations (like RomulusC and TNG50) have allowed for unprecedented insights into the nature of the multiphase IGrM. Figure 11 demonstrates the multiphase gas structure in the ROMULUSC simulation at $\mathrm{z}=0.31$. The top row shows $5 \times 5 \mathrm{Mpc}\left(9.5 \times 9.5 R_{500}\right)$ projections of gas density, temperature, and metallicity. The bottom row shows synthetic X-ray emission and UV absorption maps, roughly calibrated to the sensitivity of existing instruments. This figure clearly demonstrates that X-ray emission and UV absorption studies probe highly complementary regions of the IGrM. Additionally, it highlights the rich multiphase structure of the IGrM that exists out to the edges of the group outskirts.

We quantify the fractional breakdown of multiphase gas throughout the extended IGrM for $z=0$ snapshots of ROMULUSC, EAGLE, SIMBA, and TNG100, in Figure 12. Gas is divided into "hot" ( $\left.T \geq 10^{6} \mathrm{~K}\right)$, "warm" $\left(10^{5} \mathrm{~K} \leq T<10^{6} \mathrm{~K}\right)$, "cool" $\left(10^{4} \mathrm{~K} \leq T<10^{5} \mathrm{~K}\right)$, and "cold" ( $\left.T \leq 10^{4} \mathrm{~K}\right)$. The IGrM is clearly dominated by the hot phase all the way out to and beyond $4 R_{500}$, and the cooler phases are not dramatically different between the 4 simulations. SIMBA has the least amount of multi-phase gas, and by total mass even less inside $R_{500}$, since the IGrM is most evacuated in this simulation. ROMULUSC has the most warm-hot gas at large radii, but far less than at $z=0.31$ where Butsky et al. [19] showed more multi-phase gas that was disrupted by a 1:8 merger [72]. The properties of this hot inner region are intimately tied to the feedback from the central BGG. In particular, the temperature and entropy of the IGrM near the BGG depends on the recent AGN activity. After periods of relatively low AGN activity, the entropy profile of the inner $0.04 R_{500}$ is low and susceptible to thermal instability, which fuels star formation and AGN activity. After vigorous AGN activity, the inner $0.2 R_{500}$ develops hotter temperatures and a steeper entropy profile [72]. The metallicity of the hot phase is around $0.3 \mathrm{Z}_{\odot}$ and remains relatively constant throughout the inner region [19]. The prevalence and uniformity of metals in the hot phase implies that the hot phase was primarily enriched over time by diffuse gas in the group outskirts and mergers with group satellites (e.g., [111,287]).

The statistical samples of EAGLE, SIMBA, and TNG100 show more similar patterns of cool gas inside $R_{500}$ including a fractional increase inside $0.25 R_{500}$, which we detail below. Further out, warm gas begins to constitute a substantial fraction of the IGrM mass and beyond $\sim 2.5 R_{500}$, but unlike the smooth, large volume-filling hot phase, the cool gas distribution is patchy and traces gas that is stripped from satellite galaxies as they move through the IGrM. In the most extreme cases, the satellite galaxies and their stripped tails are referred to as "jellyfish galaxies" as seen in ROMULUSC O VI and H I maps in Figure 11. In ROMULUSC, gas stripped from satellite galaxies tends to be cooler and more metal-enriched than the ambient IGrM gas in the group outskirts, leading to a wider distribution of metallicities traced by cool and warm gas. ROMULUSC satellite galaxy CGMs (i.e., gas within $\lesssim 150 \mathrm{kpc}$ of satellites) show decreasing covering fractions of O VI, $\mathrm{C}$ VI and $\mathrm{H}$ I at lower IGrM radii with a significant decline inside $3 R_{500}$ [19]. Eventually, the stripped halo gas mixes with the rest of the IGrM. Constraining this mixing rate will be 
important for understanding how galaxies lose their gas and how the group environment is important for galaxy evolution.

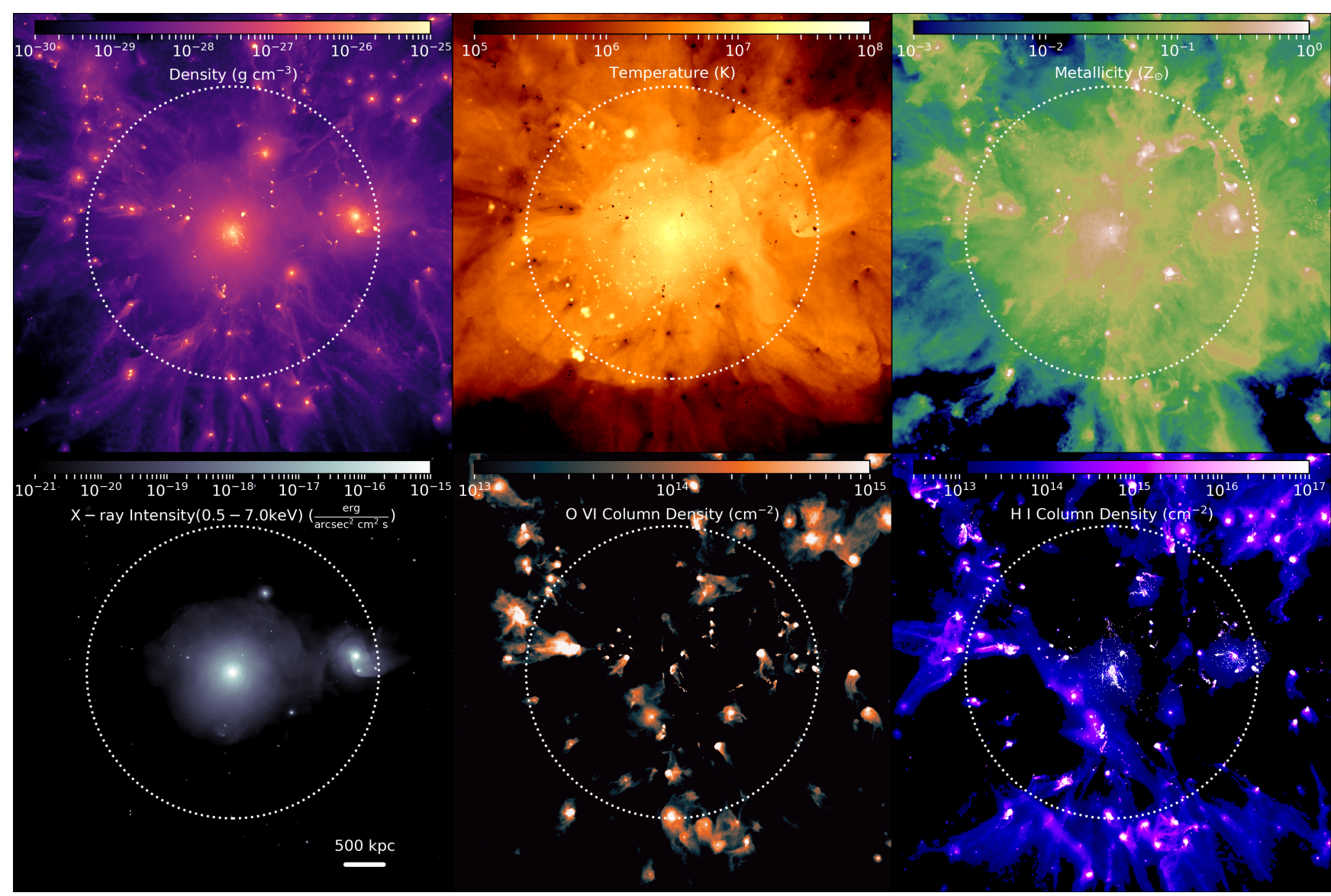

Figure 11. The ROMULUSC simulation at $\mathrm{z}=0.31$ [19]. The top row shows the projected density, temperature, and metallicity. The bottom row shows the predicted X-ray emission as well as the O VI and H I column densities. Each image spans $5 \mathrm{Mpc}$ across and the white dashed circle has a radius of $3 R_{500}$. X-ray emission probes the inner hot, dense region of the IGrM. UV absorption of ions like O VI and H I provide a highly complementary view of the IGrM, tracing the filamentary structure of cool and warm gas as it is stripped from its host galaxies.

In addition to being stripped from satellite galaxies, cool gas can also form through thermal instabilities, which is prevalent at a $\sim 10^{-2}$ fraction in the inner $0.25 R_{500}$ of most simulations of $\sim 10^{14} \mathrm{M}_{\odot}$ groups. When the local cooling time $\left(t_{\text {cool }}\right)$ of gas is roughly less than $10 \times$ the gravitational freefall time $\left(t_{\mathrm{ff}}\right)$ [288], small perturbations can seed a runaway cooling effect through which cool gas condenses out of the background medium and precipitates onto the central BGG. This process of local thermal instability in a globally stable atmosphere is self regulating and maintains a global cooling to freefall time ratio $\left(t_{\text {cool }} / t_{\text {ff }}\right) \simeq 10-20$ throughout the IGrM (e.g., $\left.[289,290]\right)$. Any gas with $t_{\text {cool }} / t_{\text {ff }} \leq 10$ will form cool filaments, lowering the density (and cooling time) of the remaining hot IGrM. This cool gas is accreted onto the central BGG and can trigger stellar or AGN feedback, driving the baryonic feedback cycle and maintaining global thermal stability in the IGrM. This process of thermal instability promoting the formation of clumps of cool gas was traced by Nelson et al. [291] using TNG50, which we check show very similar fractional values as TNG100 for the two most massive group halos in that $50^{3} \mathrm{Mpc}^{3}$ box.

Finally, we show the mass trend of fractional IGrM phases for changing group halo mass bins in the subpanels on the bottom right of Figure 12 for TNG100. The uptick in cool gas inside $0.25 R_{500}$ goes from $2 \%$, to nearly $20 \%$, to over $30 \%$ as one progresses down the mass scale from $M_{500}=10^{13.9}$ to $10^{13.1} \mathrm{M}_{\odot}$. Thus it should not be surprising that ions like $\mathrm{HI}$, Si III, and C IV in halo gas around relatively isolated ETGs that may occupy $\sim 10^{13} \mathrm{M}_{\odot}$ 
halos [286,292]. However, surveys targeting more massive groups show very little cool

(H I) or warm (O vI) gas inside $R_{\text {vir }}$ [27].
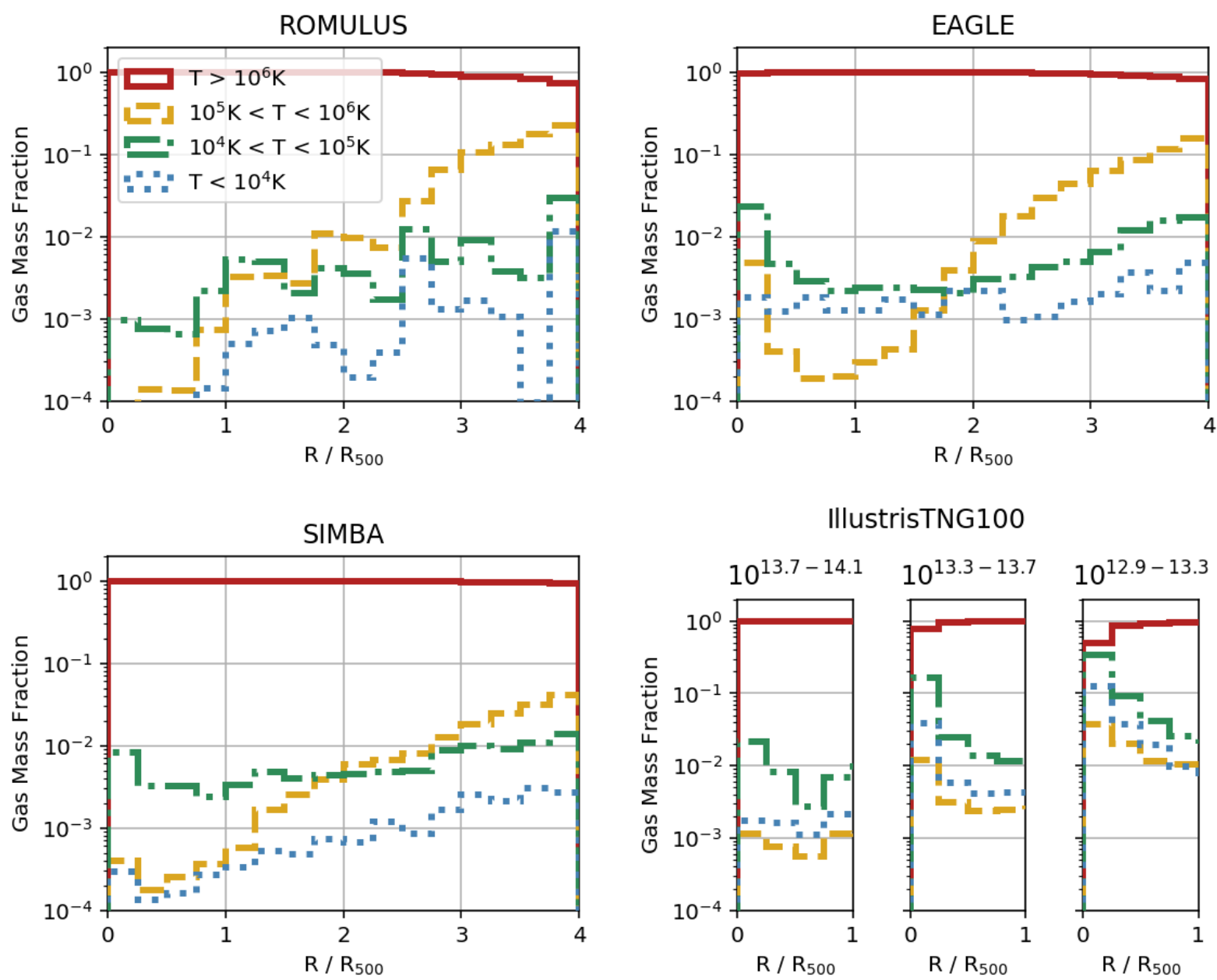

Figure 12. The total mass fraction of hot $\left(T \geq 10^{6} \mathrm{~K}\right)$, warm $\left(10^{5} \mathrm{~K} \leq T<10^{6} \mathrm{~K}\right)$, cool $\left(10^{4} \mathrm{~K} \leq \mathrm{T}<10^{5} \mathrm{~K}\right)$, and cold $\left(T \leq 10^{4} K\right)$ gas as a function of distance from the group center at $z=0$ for halos at $M_{500} \approx 10^{13.9} \mathrm{M}_{\odot}$ for ROMULUS (ROMULUSC in this case), EAGLE ( $N=7$ groups), SIMBA $(N=39)$, and the inner regions of TNG100 for this bin (left subpanel, $N=13$ ) and two lower mass halo bins (middle, $M_{500}=10^{13.3-13.7} \mathrm{M}_{\odot}, N=41$; \& right, $M_{500}=10^{12.9-13.3} \mathrm{M}_{\odot}$, $N=105$ ). Although hot gas dominates the mass fraction within $4 R_{500}$ of the BGG in all cases, cool gas never constitute less than $10^{-3}$ of the total gas mass within $R_{500}$.

\subsection{Satellite Galaxies in Groups}

Group satellites have been studied extensively in observational surveys, not least because groups are more than an order of magnitude more common than massive clusters [35]; even medium-size surveys such as GAMA therefore include more than a thousand of them [293]. Although each individual group contains fewer satellite galaxies than a rich cluster, collectively they still host $\gtrsim 2$ times as many satellites (see Figure 13). These observations generally place group satellites between clusters and the field with, for example, quenched fractions of $\approx 60 \%$ [11] and $\mathrm{H}$ I mass fraction ratios $\left(M_{\mathrm{HI}} / M_{\star}\right)$ of $\approx 0.15$ [30] at $M_{\star}=2 \times 10^{10} \mathrm{M}_{\odot}$, (extended) X-ray detection fractions of bright ETGs as high as $\approx 90 \%$ [294], and elliptical galaxy fractions of $\approx 50 \%$ [295].

In simulations, stripping of the (extended) warm-hot gas halos of group satellites is robustly predicted (e.g., Butsky et al. [19], Bahé et al. [296], Zinger et al. [297]), even for galaxies that are still (well) beyond the virial radius of the group. Ram pressure is strong enough to explain this gas loss, in particular for galaxies that (temporarily) move through 
a denser part of the IGrM or with a higher velocity than typical at a given radius [298]. As discussed above, this gas loss is simultaneously predicted to be an important route of IGrM enrichment [19].

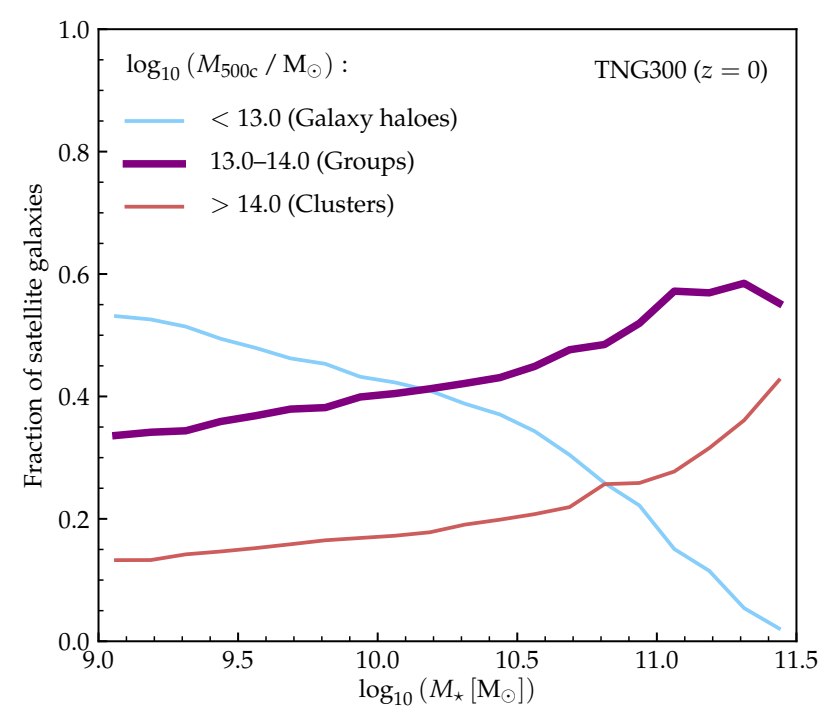

Figure 13. Fraction of satellite galaxies in the IllustrisTNG300 simulation that are hosted by galaxyscale halos $\left(M_{500 \mathrm{c}}<10^{13} \mathrm{M}_{\odot}\right.$, light blue), groups $\left(M_{500 \mathrm{c}}=10^{13}-10^{14} \mathrm{M}_{\odot}\right.$, thick purple), and clusters $\left(M_{500 c}>10^{14} \mathrm{M}_{\odot}\right.$, light red), respectively, as a function of their stellar mass. Groups host around a third of low-mass satellites, and more than half of those with $M_{\star} \approx 10^{11} \mathrm{M}_{\odot}$; their contribution exceeds that of clusters at all stellar masses shown here.

To our knowledge, no simulation that stretches up to group scales is currently able to selfconsistently model the evolution of atomic and molecular hydrogen (see e.g., Hopkins et al. [113], Applebaum et al. [299] for examples of such simulations on smaller scales). Some authors have, however, modelled $\mathrm{HI}$ in post-processing with theoretically and/or empirically motivated relations (e.g., Blitz and Rosolowsky [300], Rahmati et al. [301], Gnedin and Draine [302]) to derive $\mathrm{H} \mathrm{I}$ and $\mathrm{H}_{2}$ masses of group satellites. For EAGLE, Marasco et al. [303] demonstrated agreement with ALFALFA observations and found that $\mathrm{H}$ I loss on group scales was driven by a complex mixture of tidal stripping, ram pressure, and satellite-satellite encounters. Stevens et al. [304] created detailed H I mock observations of the TNG100 simulation, showing that this step was critical in achieving a match to the observed H I mass fractions of Brown et al. [30]. They also found, however, that the H I loss in TNG100 parallels the decline in SFR, contrary to the observations that show lower H I mass fractions even at fixed SSFR [30].

In Figure 14, we compare the $\mathrm{HI}$ deficiency of group galaxies $\Delta \mathrm{HI}=$ $\log _{10}\left(M_{\mathrm{H} \text { I,sat }} / M_{\mathrm{HI} \text {,cen }}\right)$ (where $M_{\mathrm{H} \text { I,sat }}$ and $M_{\mathrm{HI} \text {,cen }}$ are the total H I masses of group satellite and central galaxies in a narrow range of $M_{\star}$ ) as predicted by the Illustris, IllustrisTNG, and Hydrangea simulations. For the first two, we take the H I masses computed by Diemer et al. [305] on a cell-by-cell basis with the Gnedin and Draine [302] $\mathrm{H} \mathrm{I} / \mathrm{H}_{2}$ partition; for Hydrangea the $\mathrm{H}$ I have been computed in analogy to Bahé et al. [306] with the empirical Blitz and Rosolowsky [300] $\mathrm{HI} / \mathrm{H}_{2}$ partition. Despite the variety of simulations, resolutions, and $\mathrm{H}$ I models, the predictions are remarkably uniform: all simulations (except for Illustris) predict an $\mathrm{HI}$ deficiency of $\Delta \mathrm{HI} \approx 0.9$ at the low-mass end $\left(M_{\star} \approx 10^{9.5} \mathrm{M}_{\odot}\right)$, and a less extreme difference at high masses $(\Delta \mathrm{H} \mathrm{I} \approx 0.2)$.

Stevens et al. [307] investigated the molecular $\left(\mathrm{H}_{2}\right)$ masses of satellite galaxies in TNG100 through a similar mock imaging approach as Stevens et al. [304]. Despite the lack of a directly modelled cold ISM phase in these (and other) simulations, they obtained an $\mathrm{H}_{2}$ mass fraction for group satellites that is $\approx 0.6$ dex lower than in the field, consistent with data from the xCOLD GASS survey [308]. 


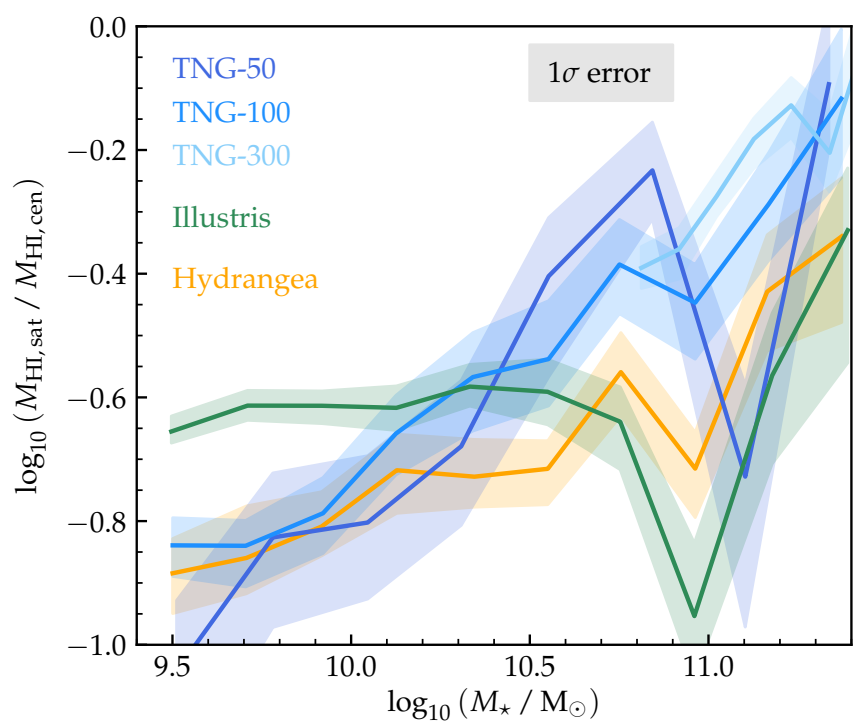

Figure 14. Atomic hydrogen ( $\mathrm{HI}$ ) deficiency of group satellites compared to centrals of the same stellar mass, as predicted by the Illustris (green), IllustrisTNG (shades of blue), and Hydrangea (orange) simulations (computed following Diemer et al. [305] and Bahé et al. [306], respectively). Solid lines show the difference between mean $\mathrm{H}$ I masses in each bin, shaded bands the corresponding $1 \sigma$ uncertainties obtained from bootstrapping. All simulations predict H I-deficient group satellites, with the difference generally largest (almost 1 dex) for the least massive galaxies. Note that for IllustrisTNG300 (light blue), H I masses are only computed for all galaxies with $M_{\star}>5 \times 10^{10} \mathrm{M}_{\odot}$.

The impact of this gas loss on star formation is arguably the most well-studied aspect of simulation works on group galaxies. Unanimously, simulations predict that the quenched (or red) fractions of group galaxies are significantly higher than for equal-mass field galaxies (e.g., Donnari et al. [34], Bahé et al. [42], Tremmel et al. [49]). In simulation suites that include groups, as well as more massive clusters (Hydrangea/C-EAGLE, IllustrisTNG), clear trends with halo mass are seen, at least for $M_{\star} \lesssim 10^{11} \mathrm{M}_{\odot}$ (see Figure 15): quenched fractions in groups are below those for clusters by up to a factor of $\approx 2[34,42]$. When only considering satellites that were directly accreted onto their $z=0$ host and not quenched already, however, Donnari et al. [34] report that this trend reverses for $M_{\star} \gtrsim 3 \times 10^{10} \mathrm{M}_{\odot}$ : at the massive end, the quenched fraction of this subset of satellites is highest for groups (up to $80 \%$ ) and lowest in massive clusters (40\%). Donnari et al. [34] interpret this "host rank inversion" as a result of AGN feedback: while this still operates efficiently for massive satellites in groups, it is suppressed in more massive clusters and therefore does not quench massive galaxies as efficiently.

A second indicator of changes to the baryon cycle in group galaxies, their ISM metallicity, was investigated by Genel [309], Bahé et al. [310], and Gupta et al. [311] with the Illustris, EAGLE, and TNG100 simulations, respectively. All three studies found elevated metallicities of satellites compared to the field, a difference that is more pronounced for lower $M_{\star}$ and higher halo mass, in qualitative agreement with observations [312]. For EAGLE, Bahé et al. [310] showed that this enhancement also agrees quantitatively with the observations, and is also predicted for stellar metallicities. Together, these studies identified three mechanisms that contribute to the elevated metallicities. Firstly, ram pressure stripping removes predominantly gas at larger radii, where the metallicity is lower $[309,310]$. Secondly, suppressed inflows of pristine gas within satellites [313] prevent the dilution of the ISM [310]. Finally, Gupta et al. [311] showed that the gas that is still replenishing the satellite ISM has higher metallicity than for isolated galaxies. Both TNG and EAGLE 
predict that this enhancement is not restricted to satellites within the virial radius of their group, but already affects galaxies during their infall [310,311].

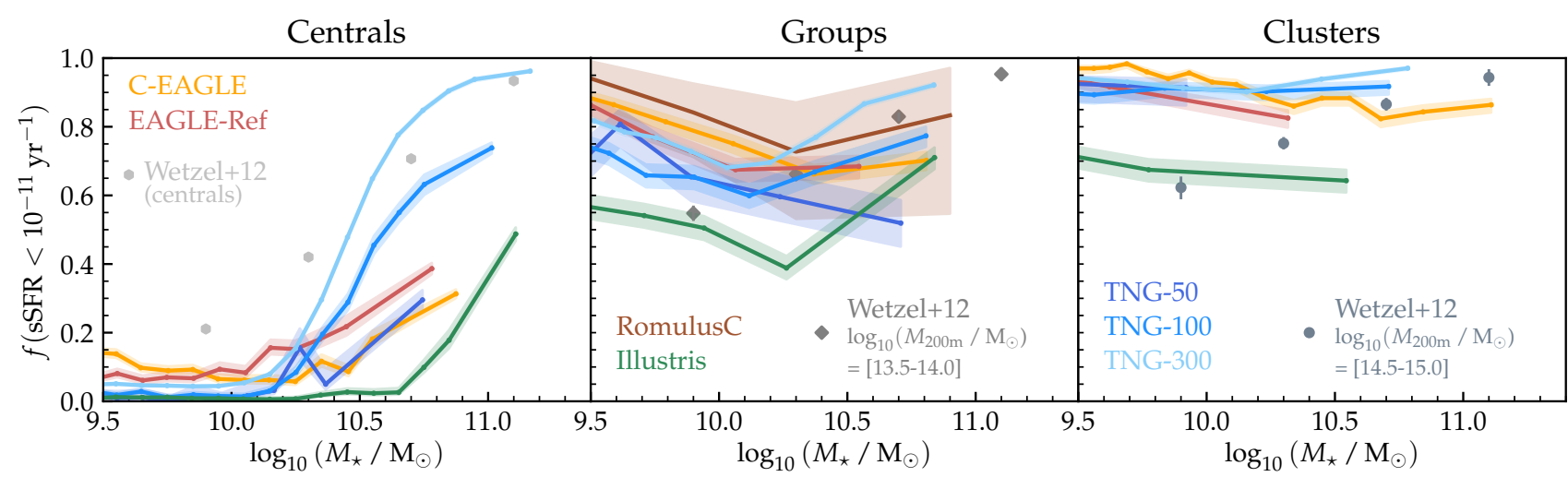

Figure 15. Quenched fractions $f_{q}$ of central galaxies (left) and of satellites in groups $\left(M_{500}=10^{13}-10^{14} \mathrm{M}_{\odot}\right.$, (middle)) and clusters $\left(M_{500}>10^{14} \mathrm{M}_{\odot}\right.$, (right) $)$ as predicted by different simulations. For (C-)EAGLE, Illustris, and IllustrisTNG, these are computed directly from the simulation outputs; the quenched fractions for RomulusC are taken from Tremmel et al. [49]. Especially on the low stellar mass end $\left(M_{\star} \lesssim 2 \times 10^{10} \mathrm{M}_{\odot}\right)$, different simulations agree closely for centrals $\left(f_{q} \approx 0\right)$ and clusters $\left(f_{q} \approx 1\right)$, whereas predictions on group scales show a much larger diversity $\left(f_{q}\right.$ between 0.6 and 1$)$. Observational data from Wetzel et al. [11] are shown for approximate guidance, but neither the satellite selection nor the host mass ranges are matched to their analysis.

While ram pressure is acting on group galaxies, it distorts their gas into long "jellyfish" tails. In TNG100, these have been studied by Yun et al. [23] through visual inspection. Depending on group mass, these authors found that $\approx 25-45 \%$ of gas-bearing satellites show evidence of such tails in their (total) gas density maps, only moderately lower than the equivalent fraction for clusters $(\approx 65 \%)$. Even when considering only the (observable) $\mathrm{H} \mathrm{I}$ component in TNG100, Watts et al. [314] find statistically significant asymmetries that visually resemble observed $\mathrm{H}$ I tails (e.g., Chung et al. [315]), with a slightly higher occurrence (21 vs. $28 \%$ ) amongst group galaxies compared to those in lower-mass halos.

While the stripping of gas is the clearest predicted effect of ram pressure, simulations have also begun revealing second-order effects due to the compressive effect on the leading edge of group satellites. In RomulusC, for instance, Ricarte et al. [316] demonstrated a correlation between ram pressure and black hole accretion rates as well as star formation rates, evidence for which has also been seen in recent observations [317]. A compressioninduced enhancement of star formation has also been described in the EAGLE simulations by Troncoso-Iribarren et al. [318]. A caveat applicable to both simulations, however, is that stars are formed directly from the tenuous ISM phase rather than from dense molecular gas that may have a different susceptibility to the effect of ram pressure, at least in detail.

The high resolution of contemporary galaxy group simulations has also enabled studies of their (stellar) morphology. Feldmann et al. [319] studied the transformation from disc to elliptical galaxy morphologies in one zoom-in simulation, and identified major mergers prior to accretion as the key driver of this change. More recently, Joshi et al. [320] presented a detailed analysis of galaxy morphology in groups and low-mass clusters from the TNG50 and TNG100 simulations. They found that up to 95\% of (satellite) disc galaxies are transformed into non-discs by $z=0$, with redistribution of stars by tidal shocks during pericentric passages as the dominant mechanism behind the transformation.

Even more fundamentally, simulations have investigated the tidal stripping of dark matter and stars from group satellites. With the caveat that this stripping may be artificially enhanced by numerical artefacts (van den Bosch and Ogiya [321], but see Bahé et al. [31]), the clear prediction is that dark matter stripping far outweighs that of stars: for example, Joshi et al. [77] found in an individual zoom-in simulation of a galaxy group that stellar stripping at a $>10 \%$ level typically only occurs after the loss of $\approx 80 \%$ of their dark mat- 
ter halo. This relatively minor role of stellar stripping, also borne out by EAGLE [310] and IllustrisTNG [322], leads to a stellar-to-halo mass relation with a much higher peak ratio $(\approx 0.15)$ for group satellites than centrals $(\approx 0.02)$. The most extreme form of satellite stripping, their complete disruption, is even predicted to be somewhat more common in groups than clusters ( $\approx 65$ vs. $50 \%$ of all accreted satellites with a total mass of $\sim 10^{12} \mathrm{M}_{\odot}$ ), due to the higher efficiency of dynamical friction driving satellites towards their dense centers [31].

Finally, we note that simulations are also increasingly demonstrating the importance of groups for the evolution of cluster satellites: around $50 \%$ of all $z=0$ satellites in massive clusters of the TNG300 simulation were quenched in a group before being accreted onto their final host [34]; Pallero et al. [323] came to a similar conclusion with the Hydrangea/CEAGLE simulations. Similarly, Jung et al. [324] found that almost half of all $M_{\star} \gtrsim 10^{9} \mathrm{M}_{\odot}$ galaxies accreted onto clusters as (group) satellites are already gas poor at the time of cluster infall, compared to only $6 \%$ of central galaxies. Even where groups and their galaxies have not (yet) joined a cluster, they therefore contribute to the large-scale environmental dependence of galaxy properties far beyond the "edge" of the cluster [325].

\subsection{Simulating the Impact of Galaxy Group Astrophysics on Large-Scale Structure Cosmology}

Measurements of the growth of large-scale structure (LSS) can provide powerful tests of our cosmological framework [326-330]. Importantly, they are independent of, and complementary to, constraints from analyses of fluctuations in the cosmic microwave background (CMB) and geometric probes, such as Type Ia SNe and baryon acoustic oscillations (BAOs). Generally speaking, the different LSS tests (e.g., Sunyaev-Zel'dovich power spectrum, cosmic shear, group and cluster number counts, redshift space distortions, etc.) are just different ways of characterising the 'lumpiness' of the matter distribution on different scales. On very large scales, perturbation theory is sufficiently accurate to calculate this distribution reliably. However, most existing LSS tests probe well into the non-linear regime. The standard approach is therefore either to calibrate the 'halo model' (e.g., HMcode package; [331]) using large N-body cosmological simulations, or to use such simulations to correct linear theory empirically (e.g., HALOFIT package; [332]).

If the matter in the universe were composed entirely of dark matter, these approaches would likely be sufficient. However, baryons contribute a significant fraction of the matter density and work by a number of different groups has shown, using cosmological hydrodynamical simulations, that feedback processes associated with galaxy formation can have a significant effect on the matter distribution on scales of up to a few tens of megaparsecs [66,333-335]. Therefore, while such effects are typically ignored or treated in a simple way as a first step when modelling LSS data, it is of critical importance to understand their impact, as they can introduce significant biases in the inferred cosmological parameters in upcoming surveys if no action is taken (e.g., [70,336,337]).

Galaxy groups (taken here to be bound systems with total masses of $\sim 10^{13-14} \mathrm{M}_{\odot}$ ) play a particularly important role in LSS cosmology. This is simply because a sizeable fraction of the galaxies, baryons, and overall matter in the Universe resides in groups. Consequently, LSS cosmology tests that probe more 'typical' environments (such as cosmic shear, galaxy-galaxy lensing, galaxy clustering, redshift space distortions, CMB lensing, etc.), as opposed to tests that sample only the most massive systems (such as the SZ effect power spectrum and current cluster count surveys), will be sensitive to the abundance of galaxy groups and the spatial and kinematical distributions of matter within and around them.

A quantitative demonstration of the importance of the galaxy groups on LSS cosmology can be provided by examining the contribution by halo mass to the total matter power spectrum, $P(k)$. Note that, at present, virtually all current LSS tests probe cosmology through its effects on the matter power spectrum. In the left panel of Figure 16 we show the contribution to the dimensionless matter power spectrum by halos of different mass, as calculated in Mead et al. [338] using the HMCODE halo model. Note that the dimensionless matter power spectrum, $\Delta^{2}(k)$, is related to $P(k)$ via a multiplicative factor 
$4 \pi(k / 2 \pi)^{3}$ and $k$ is the wavenumber related to the comoving size scale $(\lambda)$ by $k=2 \pi / \lambda$. The curves show the resulting power spectrum when integrated up to different choices for the maximum halo mass. The results demonstrate that halos corresponding to galaxy groups contribute the majority of the power on the scales relevant for most LSS probes (typically $k \lesssim 10 \mathrm{hMpc}^{-1}$ ). This conclusion is consistent with previous simulation-based findings presented in van Daalen and Schaye [339].

A clear ramification of groups contributing a large fraction of the signal to current LSS tests of cosmology is that theoretical models/simulations must be able to predict the abundance of groups and the matter distribution within them to a very high level of precision on average. For example, the upcoming Rubin Observatory (formerly LSST), Euclid, and Roman Space Telescope surveys are expected to measure the matter power spectrum to better than a few percent accuracy over a very wide range of scales, implying that the theoretical uncertainties in predicting $P(k)$ should be smaller than this to avoid biasing cosmological parameter constraints (e.g., Huterer and Takada [340], Hearin et al. [341]). As already noted, because baryons contribute a non-negligible fraction of the matter density, this means an accurate theoretical description of the baryons within groups (and their back reaction on the dark matter) is also required.

Given the complexity of the physical processes involved in setting the thermodynamic properties of the IGrM and the difficulty in simulating the full range of scales at play (see Section 3), the prospects for accurately (to typically percent level) describing the impact of baryons and group astrophysics on LSS would at first sight seem daunting, if not altogether hopeless at present. Indeed, previous simulation work has shown that variations of the parameters associated with the efficiencies of feedback processes even within plausible bounds can lead to relatively large differences in the predicted properties of groups (e.g., $[7,132,133,342])$. Variations in resolution and method of solving the hydrodynamic equations may also produce important changes (e.g., [84]), though they are arguably of secondary importance compared to changes in the subgrid modelling associated with feedback (e.g., [85]). A consequence of these large simulation-to-simulation variations in the predicted properties of groups are relatively large study-to-study variations in the predicted impact of baryons on the matter power spectrum (see the simulation comparisons in $[343,344])$.

The study-to-study variation in the predicted properties of groups and the impact of baryons on $P(k)$ is not unexpected. It is a consequence of not being able to derive the efficiencies for the relevant feedback processes from first principles (see discussion in [39]). This problem is made particularly challenging in the context of simulations with finite resolution and approximations for other (coupled) physical phenomena. As we cannot derive the efficiencies from first principles, the feedback in simulations must be calibrated in order to ensure they reproduce particular quantities, after which the realism of the simulations can be tested against other, independent quantities. For LSS cosmology, the main problem we are trying to solve is to accurately model the impact of baryons on $P(k)$. One way this could be achieved is to directly measure $P(k)$ from observations (e.g., via cosmic shear or galaxy-galaxy lensing and galaxy clustering) and compare this with the $P(k)$ predicted in the absence of baryon physics to measure the impact of baryons. However, such an approach is generally a non-starter, as to predict $P(k)$ in the absence of baryons requires that we assume a cosmology and therefore the process of deriving the impact of baryons on $P(k)$ becomes explicitly dependent on cosmology and we would have adopted a circular line of reasoning in our aim to constrain cosmology with LSS measurements. 

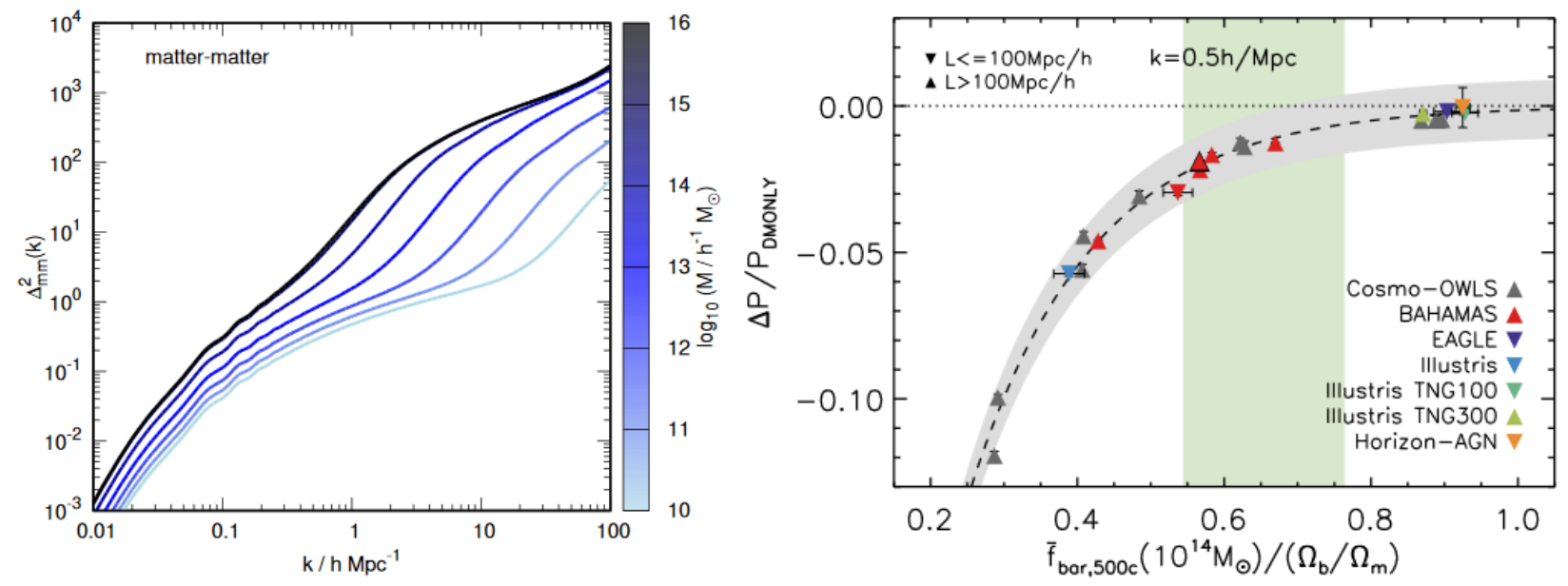

Figure 16. (Left) The contribution to the dimensionless matter power spectrum for halos of different mass. The curves show the resulting power spectrum when integrated up to different choices of the maximum halo mass. Here we can see that halos corresponding to galaxy groups $\left(10^{13-14} \mathrm{M}_{\odot}\right)$ contribute a very large fraction of the power over the range of wavenumbers probed by LSS measurements (typically $k \lesssim 10 \mathrm{hMpc}^{-1}$ ). This figure was reproduced with permission from Mead et al. [338]. (Right) The effect of galaxy formation on the matter power spectrum at the scale $k=0.5 \mathrm{hpc}^{-1}$ as a function of the mean normalized baryon fraction in $\sim 10^{14} \mathrm{M}_{\odot}$ halos. Shown are simulations from cosmo-OWLS and BAHAMAS (grey and red), EAGLE (purple), Illustris (blue), TNG100 and TNG300 (cyan and green) and Horizon-AGN (orange). Baryon fractions were calculated within $r_{500 c}$ for halos in the mass range $M_{500 c}=\left[6 \times 10^{13}, 2 \times 10^{14}\right] \mathrm{M}_{\odot}$. The dashed curve shows that at this $k$, a simple exponential function of the baryon fraction fits the predictions for the suppression of power of all simulations to within $1 \%$ (grey band). The vertical green band shows a range of mean group-scale baryon fractions roughly consistent with observations. These results demonstrate that the differences in the simulation predictions for the impact of baryons on $P(k)$ can be understood to high accuracy based on differences in the baryon fraction on the group scale and that calibration to observed baryon fractions is a promising tool for constraining feedback processes in the simulations. This figure was reproduced with permission from van Daalen et al. [344].

An alternative approach is to modify the gravity-only predictions with simple, physically-motivated prescriptions for baryon physics that have some number of associated free parameters and to jointly constrain the cosmological and feedback parameters through comparisons to LSS observables. Examples of this approach include the halo model approach HMCODE of Mead et al. [331,338] and the 'baryonification' approaches (which directly modifies the outputs of gravity-only simulations) of Schneider and Teyssier [334], Schneider et al. [345] and Aricò et al. [346]. The advantages of these approaches include: (i) they are considerably cheaper than running full cosmological hydrodynamical simulations; (ii) the baryon prescriptions are generally flexible and easy to adjust/improve; (iii) they can be straightforwardly incorporated within existing pipelines based on gravity-only simulations or the halo model. The disadvantage of these approaches are that the modeling of baryon physics and its back reaction on dark matter is simplistic and generally not self-consistent and that, unless the impact of baryons is very different than the cosmological variations being explored, one expects there to be important degeneracies between the cosmological and feedback 'nuisance' parameters and a degrading of cosmological constraining power (due to marginalization over uncertain baryon physics).

What about using cosmological hydrodynamical simulations directly for LSS cosmology predictions? The approach of the BAHAMAS program [41,347] (see also the recent FABLE simulations; Henden et al. [44]), is to explicitly calibrate the feedback efficiencies so that they reproduce the observed baryon fractions of galaxy groups. Except for an explicit dependence on the universal fraction, $\Omega_{b} / \Omega_{m}$, the baryon fractions of groups should be insensitive to changes in cosmology [348] and therefore they represent a highly useful metric on which to calibrate. Furthermore, since the growth of matter fluctuations is fundamentally a gravitational process, by ensuring the simulations have the correct baryon fractions on the scale of groups and gravitational forces are computed self-consistently, 
the impact of baryons on $P(k)$ should be strongly constrained by this calibration approach. Indeed, van Daalen et al. [344] have recently shown that one can understand the differences in the predicted impact of baryons on $P(k)$ from different simulations at the percent level in terms of the differences in baryon fraction in the various simulations at a mass scale of $\sim 10^{14} \mathrm{M}_{\odot}$ (see right panel of Figure 16). It is worth noting here that the simulations analysed in that study varied in resolution by more than a factor of 1000 in mass, used different hydro solvers, and assumed different baseline cosmologies.

The implication of this recent development is that, through calibration of feedback efficiencies on the observed baryon fractions of galaxy groups, we strongly limit the ways in which feedback can affect $P(k)$ (in other words we have a very strong prior on the impact of baryons). This, in turn, means much stronger (and more robust) cosmological constraints from LSS. However, before claiming victory, a number of important issues require further attention. Firstly, as the calibration is reliant on observations of galaxy groups, the uncertainties in the observed baryon fractions need to be properly included in any cosmological analysis. It goes without saying that the selection function of the group calibration data set must be also reasonably well understood and accounted for (otherwise we risk miscalibrating the feedback). In addition, while the results of van Daalen et al. [344] look very promising, we need to explicitly verify that different kinds of simulations (e.g., that vary how feedback, star formation, and so forth are implemented, resolution, how the hydro equations are solved, etc.) that are calibrated in the same way to the same precision actually produce the same $P(k)$. In other words, we need to check whether differences in the details and evolution of the simulations affect the end state (e.g., $P(k)$ at $z=0$ ) if some aspect of that end state has an imposed boundary condition (the baryon fractions at $z=0$ ). We also have precious few observational constraints on the baryon fractions of groups beyond $z \sim 0.3$ and it is therefore unclear to what extent the simulations calibrated at $z=0$ remain 'well behaved' at significantly earlier times. Finally, the level of degeneracy between the various cosmological parameters and the parameters governing feedback efficiency needs to be fully quantified. As already noted, there is an explicit dependence on the universal baryon fraction, $\Omega_{b} / \Omega_{m}$, but there may well be other less obvious degeneracies that require understanding in order to obtain percent level constraints on parameters such as the dark energy equation of state. Thus, while a promising start has been made in addressing this complex challenge, a number of important steps remain in order to fully account for the impact of group astrophysics on high-precision LSS cosmology.

Finally, we note that, in this section, we have discussed the impact of group astrophysics on LSS cosmology, with a focus on the non-linear matter power spectrum, $P(k)$, which is the basis of many precision LSS probes of cosmology including cosmic shear, CMB lensing, galaxy clustering, and so on. We have not specifically discussed the impact of group astrophysics on attempts to use the abundances (number counts) of galaxy group themselves to constrain cosmology. Of course, one advantage that groups have over clusters in this regard is that they are much more numerous, potentially allowing for stronger cosmological constraints than what might be obtained by clusters alone (e.g., [349-352]). The challenge is that they are more difficult to detect and to model, as already discussed. These issues are particularly pertinent for group counts, as they affect the cosmological observable in a much more direct way than, for example, cosmic shear. Nevertheless, as our observational picture and ability to model galaxy groups improves, we expect galaxy group counts to play an increasingly important probe of cosmology and one that is complementary to constraints coming from cosmic shear, CMB lensing, and other LSS probes. 


\section{Future Directions}

\subsection{Using Simulations to Make Predictions for Existing and Future Missions and Telescopes}

Cosmological simulations are widely used to make predictions for future missions, often in such a capacity that a mission's approval or rejection may hinge on these predictions. It is therefore incumbent upon simulators to generate mock observations that do not suffer from numerical effects, poorly implemented modules, or insufficient resolution. However, simulation predictions that are later refuted are not necessarily the result of numerical problems: instead, such discrepancies may also reveal new physical processes. This applies in particular to the complex interplay of non-gravitational physics and dynamics in groups.

A near-term example relevant for groups covers predictions of $\mathrm{X}$-ray line emission from the EAGLE simulation that should be observed by several future missions, including XRISM to be launched in 2022. Figure 17 from Wijers et al. (in prep) demonstrates that XRISM should be able to detect O VII and Mg XII emission tracing the approximate temperatures of virialized IGrM gas. This prediction follows on from the "virial temperature thermometer" model of Oppenheimer et al. [76] and Wijers et al. [353] that specific metal ions should trace the volume-filling virialized halo gas corresponding to the temperature of the ion's peak collisional ionization fraction. While (UV-band) O VI traces outer virialized galactic halo gas at $3 \times 10^{5} \mathrm{~K}, \mathrm{O}$ VII and $\mathrm{O}$ VIII in the X-ray spectrum are predicted to trace $\geq 10^{6} \mathrm{~K}$ IGrM in groups. Figure 8 of [353] predict that $\mathrm{O}$ VIII absorption is strongest in poor groups with $M_{500} \sim 10^{13} \mathrm{M}_{\odot}$ and Fe XVII in intermediate groups with $M_{500} \sim 10^{13.5} \mathrm{M}_{\odot}$.

XRISM should measure the significant metal and (for an assumed metallicity), baryon contents of the IGrM out to $100 \mathrm{kpc}$ from the central galaxy as predicted by the EAGLE simulations in Figure 17. Micro-calorimeters on Athena and Lynx should be able to resolve the interior metal emission of the IGrM at superb $(<10 \mathrm{eV})$ spectral resolution and signal to noise ratio. If, however, XRISM does not detect O VIII or Mg XII at the levels predicted in this figure, this might point to one of the following scenarios. Firstly, the IGrM might not be as metal-enriched as EAGLE predicts, for example, because the nucleosynthetic yields assumed by the simulation are too high, or due to a higher-than-predicted fraction of metals being retained within galaxies. Secondly, EAGLE might over-predict the IGrM baryon content, and hence the gas density and emission line luminosity ${ }^{13}$. A third possibility is that metals in real groups are distributed more (or in principle also less) homogeneously than in EAGLE, which would affect the radially averaged cooling rates, and hence line emission luminosities - metal-line emission is highly sensitive to the distribution of gas density and metallicity distributions on both the macro and micro IGrM scales. Finally, nonequilibrium ionization [94] and/or dual temperature electron-ion plasmas [354] could alter simulation predictions that almost always assume ionization equilibrium and equipartition between electrons and ions.

When simulators generate mock observations, it is important to present their predictions in a manner that accurately showcases the capabilities of existing and proposed instruments. Often it is advisable to work with instrumentalists and observers. An example of an attempt to fairly compare capabilities of existing and future missions appears in Figure 18 for a TNG100 $M_{500}=10^{13.6} \mathrm{M}_{\odot}$ halo at $z=0.05$. Each panel shows a mock $100 \mathrm{ksec}$ X-ray image based on the instrument capability at launch (Cycle 0 for Chandra), but the leftmost panels show mock observations without any noise. We apply a forward modeling technique using the packages pyXSIM ${ }^{14}$ [357] and SOXS ${ }^{15}$; the SIXTE simulation software [358] is used to create the eROSITA mock. 


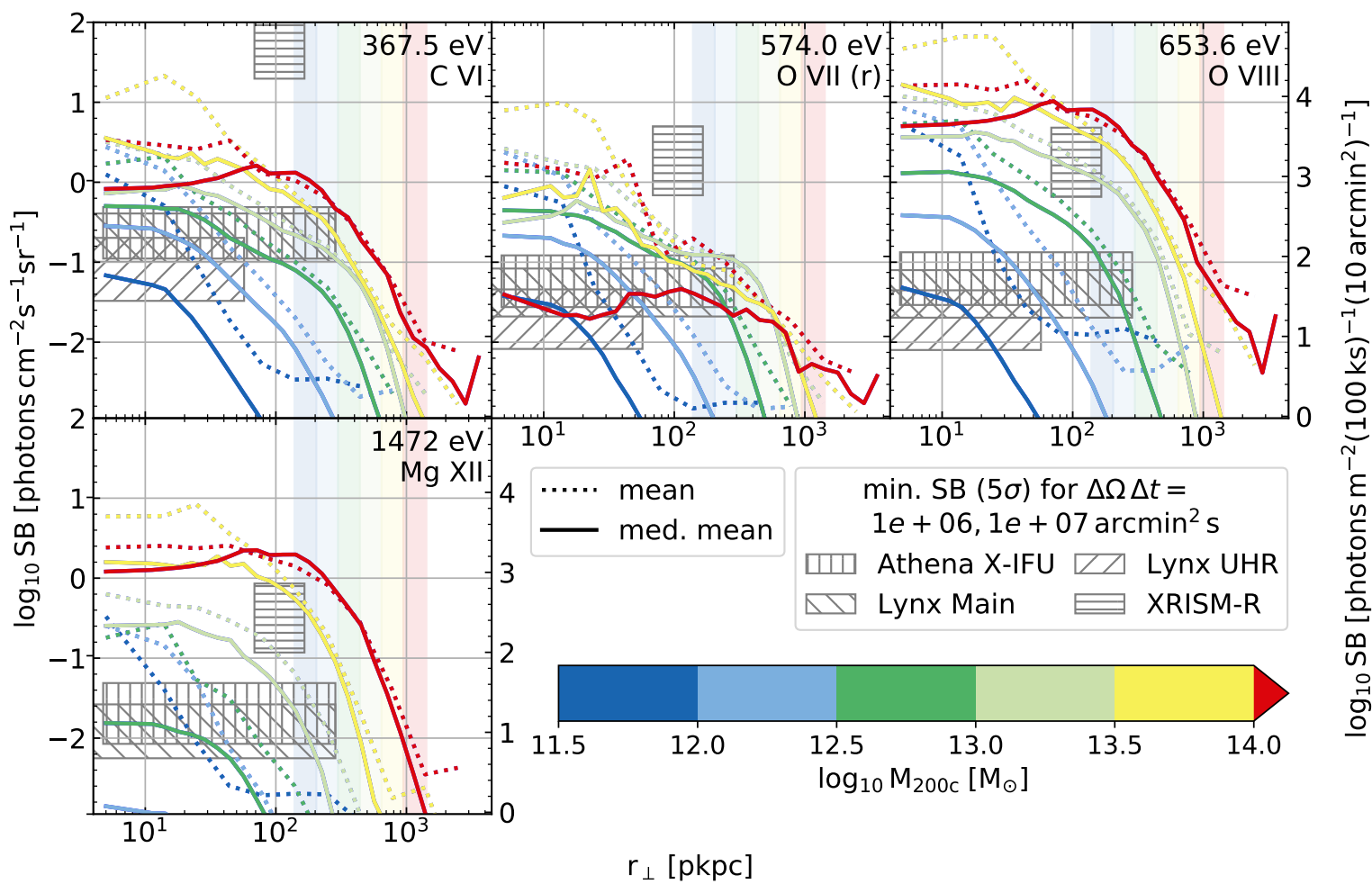

Figure 17. X-ray line emission predictions from the EAGLE simulation via Nastasha Wijers (in prep.) demonstrating the detectability by XRISM, Athena, \& Lynx space-borne micro-calorimeters as a function of impact parameter from the central galaxy. Lines are colored by halo mass with light green and yellow corresponding approximately to low-mass and high-mass groups. Faded vertical stripes indicate $R_{200}$ for each halo mass. High-mass groups are detectable by XRISM in O VIII and Mg XII at $100 \mathrm{kpc}$, while low-mass groups are detectable only in O VIII. Athena and Lynx should measure IGrM line emission for all group halos with high spatial resolution.

We choose surface brightness units (counts s${ }^{-1} \operatorname{arcmin}^{-2}$ ) to show the relative throughput of the detectors. At launch, Chandra was able to detect groups out to $R_{500}$ in $\lesssim 100 \mathrm{ksec}$, which appears consistent with the longest exposed groups of Sun et al. [2]. eROSITA can detect extended emission out to a good fraction of $R_{500}$, although it will only approach such exposures at the ecliptic poles during its eRASS:8 4-year survey [359], and will require targeted follow-up on most groups in the sky to achieve such depth. Biffi et al. [360] simulated eROSITA observations of clusters/groups between $z=0.1-2.0$ from the Magneticum Box $2 / \mathrm{hr}$ run, inputting AGN to determine if the underlying ICM/IGrM emission can be separated from AGN contamination. Oppenheimer et al. [361] simulated the stacking of galactic halos assuming $2 \mathrm{ksec}$ exposures aimed at $50 \mathrm{z}=0.01$ galaxies with an average halo mass of $M_{500}=10^{12.5} \mathrm{M}_{\odot}$ to show that the eRASS: 8 survey should be able to resolve the stacked profile out beyond $100 \mathrm{kpc}$. Their forward modeling analysis used EAGLE and TNG100 galaxies as inputs, added in noise and attempted to subtract it, and excised mock point sources from the cosmic X-ray background. However, Biffi et al. [360] and Oppenheimer et al. [361] still did not attempt to mock the scanning mode of the 8 individual all-sky surveys, instead assuming single pointed exposures with the object placed at the center, which likely under-estimates sources of systematic errors. Future data collected from $e$ ROSITA will need to be compared to simulations applying the same scanning exposures used by the eRASS:8 survey.

Continuing on the lower panels of Figure 18, Athena will be able to distinguish IGrM structure associated with the central galaxy and satellites. Finally, Lynx should be able to clearly resolve azimuthal dependence, a variety of satellite interactions, and sharp shock fronts bow shocks associated with infalling satellite as seen to the left of the simulated central. 

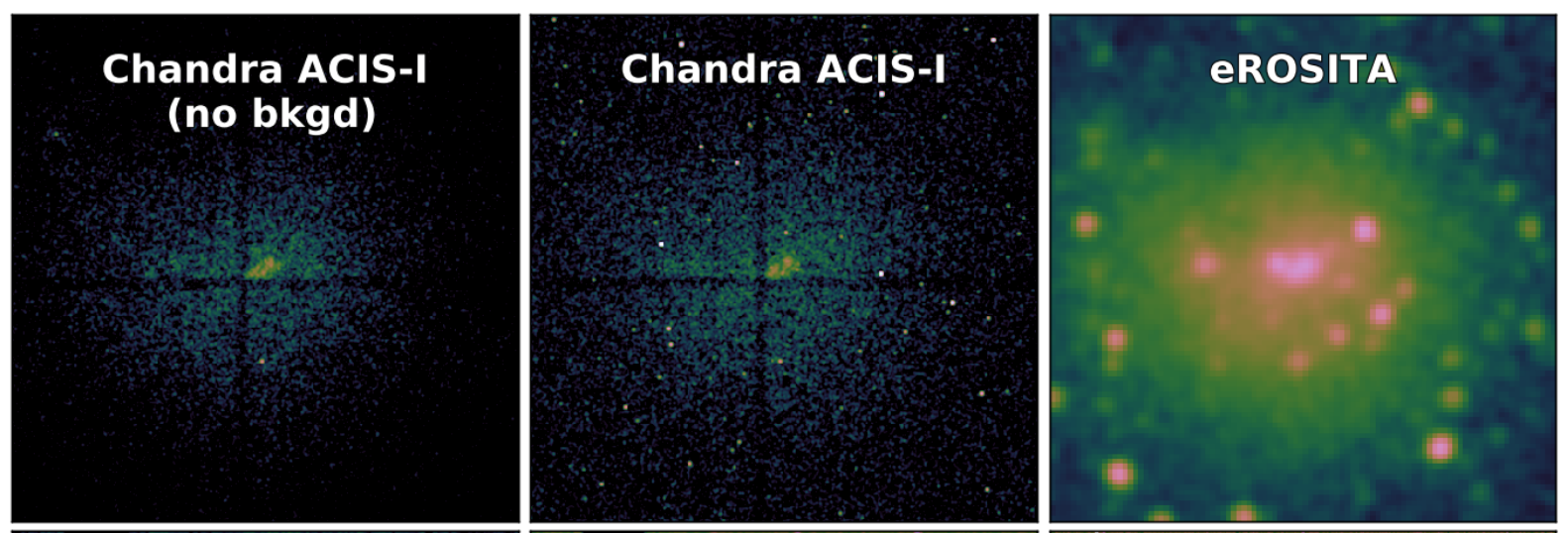

1.000
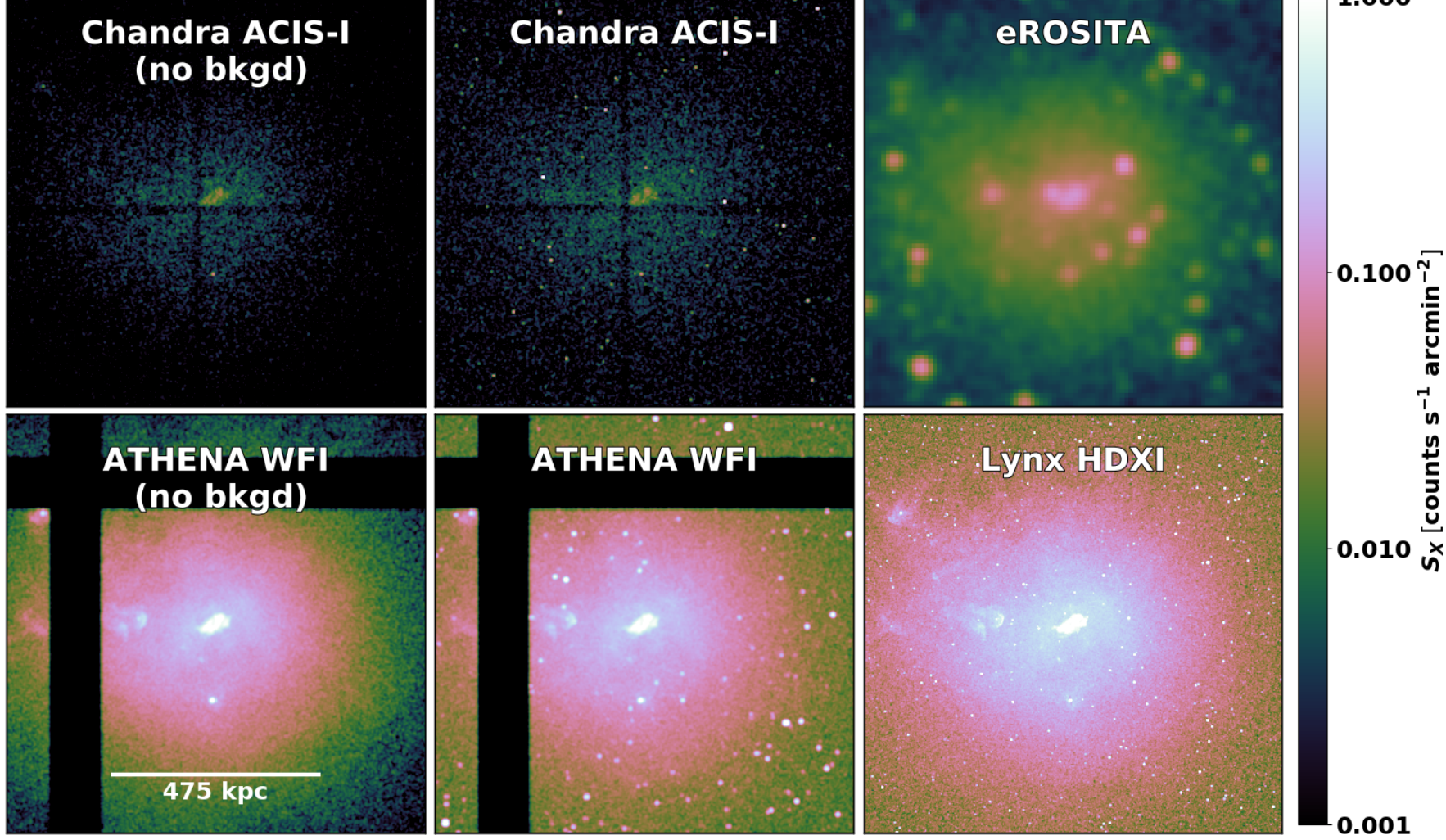

Figure 18. Simulated $100 \mathrm{ksec}$ surface brightness maps of the same TNG100 $M_{500}=10^{13.6} \mathrm{M}_{\odot}$ group placed at $z=0.05$ by CCD detectors on Chandra (Cycle 0 capability), eROSITA, Athena, \& Lynx. The two left panels show Chandra and Athena mocks without noise, while all the other panels add noise from the instrument, the Milky Way foreground, and a randomly generated cosmic X-ray background (CXB). The field of view covers out to $R_{500}$. All detectors use the color scale on the right. A. Simionescu collaborated on the production of this figure. Note: the CXB is differently randomly generated in each panel.

\subsection{Observations and Simulations in Support of Each Other}

It is clear that the increasing wealth of observational data on galaxy groups has led to continual improvement in cosmological simulations, as we gain increased knowledge about the physical processes at play and constraints on their 'efficiencies', both of which help inform the simulations. However, an important caveat to bear in mind is that observations themselves are subject to considerable uncertainties which should not be ignored when comparing with simulations, particularly if one intends to calibrate aspects of the simulations on said observations. While good strides have been made in generating realistic synthetic X-ray, thermal Sunyaev-Zel'dovich (tSZ), optical, and so forth, 'observations' of simulated groups to enable like-with-like comparisons (e.g., [7,357,362-364]), to date much less attention has been devoted to ensuring a consistent method of selection. While the use of mock catalogs is standard practice in galaxy surveys to quantify the selection function (e.g., [365-367]), the use of realistic mocks to quantify the selection function of groups selected on the basis of their hot gas properties (particularly X-ray and tSZ) has lagged behind. Instead very simplistic models (e.g., spherical beta models) are still regularly employed in characterizing the selection function. There is also often a key difference in the way observers speak about the selection function (which they normally cast in terms of observable quantities such as flux, surface brightness, or signal-to-noise ratio) and what a theorist or simulator would regard as the selection function (which is almost always with respect to halo mass). Ultimately, what is required is an iterative process involving simulations and observations, whereby mock surveys of the simulations are used to inform a consistent definition of the selection function. A like-with-like comparison is then made between the simulations and observations, shortcomings of the simulations are identified, new simulations are produced, and the cycle repeats. Each cycle yields not only an im- 
proved simulation and physical picture (hopefully), but also informs our knowledge of how biased observational methods are in selecting groups and estimating their physical parameters. Of course, the realism of the simulations must also be tested against independent observations which are not part of the calibration process (e.g., evolution of galaxy groups, environmental effects on galaxies, etc.).

While the heterogeneous nature of most pointed X-ray observations with Chandra and $X M M-N e w t o n$ do not naturally lend themselves to the development of simple selection functions, upcoming eROSITA observations should be much more tractable in this regard, given the homogeneity of the survey. Likewise, wide-field $\mathrm{tSZ}$ observations should benefit from mock surveys based on large hydrodynamical simulations, to complement existing work based on simple spatial templates (e.g., [368]), which are not expected to hold deep into the group regime [369].

\subsection{Timely Research Topics for Simulations of Groups}

Arguably, simulations of $10^{13}-10^{14} \mathrm{M}_{\odot}$ halos have so far received less attention than neighboring halo mass ranges, which might be due to the current difficulty in the observational identification and characterization of groups, as well as the complexity in their theoretical modeling. On the observational side, breakthroughs are imminent on multiple fronts: deep and highly complete spectroscopic galaxy redshift surveys such as the 4MOST Wide-Field Vista Extragalactic Survey (WAVES) will deliver robust group catalogues; IGrM probes with linear dependence on gas density - such as UV and X-ray absorption, kinetic Sunyaev-Zeldovich (kSZ), and fast radio bursts [370,371] —instead of the $\rho^{2}$ scaling of X-ray emission will map the diffuse IGrM out to large radii. Below, we therefore list a selection of timely open research topics in the field of group simulations.

- The relationship between the central SMBH and properties of the IGrM/ICM/CGM: The strong correlations between the mass of the central SMBH in a group $\left(M_{\mathrm{SMBH}}\right)$ and the temperature and X-ray luminosity $\left(T_{X}\right.$ and $\left.L_{X}\right)$ of the IGrM/ICM [372,373], provide fundamental tests of AGN feedback in simulations (see also Section 4 of the companion review by Lovisari et al. [1]). On the one hand, the $M_{\mathrm{SMBH}}$ scaling with $M_{\text {halo, }}$ assuming $T_{X}$ measures $M_{\text {halo, }}$, is a natural expectation of the SMBH growth being controlled by the binding energy of the halo [374]. The EAGLE simulation prediction that $L_{X}$ scales inversely with $M_{\mathrm{SMBH}}$ at fixed halo mass and most strongly for the CGM (i.e., galactic halo masses below the group scale; Davies et al. [162] suggests the opposite, inverse trend, which is also seen in TNG100 [140]. As the latter paper explains, the CGM $L_{X}$ is reduced in response to the integrated SMBH feedback lifting baryons out of galaxy halos, lowering the density, and significantly increasing cooling times. However, by group masses, the Gaspari et al. [372] correlations appear reproduced by TNG100 with $T_{X}\left(<R_{500}\right)$ showing surprisingly little scatter for quenched galaxies with $M_{\mathrm{SMBH}}>10^{8.2} \mathrm{M}_{\odot}$ [375]. This link between $M_{\mathrm{BH}}$ and halo-wide $T_{X}$, which is the best X-ray observational proxy for halo mass, indicates that simulations predict a fundamental relationship between $M_{\mathrm{BH}}$ and $M_{\text {halo }}$ transmitted through the virialization of halo gas. The nature of this relationship contains both the virial temperature being set by hierarchical growth of group/cluster-scale halos (as discussed by [375]), and the mechanisms of gas accretion and AGN feedback determining SMBH growth, which span scales from the SMBH radius to $R_{\text {vir }}$ (as discussed in Section 4.1 of the companion review by [78]. and in [376]). Bassini et al. [377] explored GADGET-3 cluster zoom simulations, finding that they were able to reproduce the observed $T_{500}-M_{\mathrm{SMBH}}$ and other correlations in the group and cluster regime. We emphasize the need for more simulations to explore the rich and diverse astrophysics contained in the relationship between group properties and their central SMBHs.

- Cooling flows or cold rain?: One of the key phenomenon that links the IGrM/ICM to the BGG/BCG and ultimately, to the SMBH hosted by the central galaxy is the flow of gas from the former to the latter two, particularly in CC clusters. During the cooling phase, the conventional view is that the gas typically flows inwards subsonically and en 
masse, meaning that if gas is multiphased, then all phases move inwards in a comoving fashion. This is how cooling flows were originally conceived ([378]; see also reviews by $[379,380])$; this is what pre-AGN feedback simulations found (see Figures 17 and 19 of [95]); and recently, this is how the inflow is thought to behave during times when the central AGN is quiescent (or in quadrants about the cluster center where cooling is dominant). Recent very high resolution simulations of idealized galaxy groups and clusters [150,381-386] find that when the ratio $t_{\mathrm{cool}} / t_{\mathrm{ff}}$ in a cooling group/cluster core drops below some threshold (nominally $\sim 10$ ), local density perturbations can become thermally unstable [288,290], leading to the formation of cold dense clouds. These clouds then separate from the rest of the CGM and stochastically rain down upon central galaxy and its SMBH. This "cold rain" fuels both star formation events as well as AGN outbursts.

There are number of problems with the conventional Bondi accretion model indicating that it is untenable (see, e.g., [384,385,387,388]), which the cold rain model appears to resolve. We argue that this warrants further investigation of the cold rain model within the context cosmological hydrodynamics simulations of the formation/evolution of massive galaxies, groups, and clusters. Two potential directions of study stand out: Firstly, current insights about the cold rain phenomenon come from idealized simulations that neither have satellite galaxies moving through the IGrM/ICM and inducing perturbations in their wakes, nor do they allow for interactions, like ram pressure stripping of these satellite galaxies. How these complications alter the thermal instability/cold rain picture remains unexplored. Secondly, it is not currently feasible to directly model the cold rain phenomenon in cosmological hydrodynamics simulations because that would require being able to resolve spatial scales approaching $\sim 1$ pc ( see [389] for further details). This however means that there is an opportunity for developing innovative subgrid models that can capture the most important elements of the cold rain model. There is precedence for the second option in that the torque-limited accretion subgrid model of Anglés-Alcázar et al. [153] was created to encompass idealized simulations of gas-rich accretion discs from $10 \mathrm{kpc}$ to 0.1 pc [390].

- $\quad$ New models for AGN feedback: Non-spherical, jet-like feedback appears necessary to impart energy to the IGrM/ICM while not over-evacuating the inner region, as discussed in Section 4 of the Lovisari et al. [1] companion review where they show in their Figure 7 results from an idealized simulation by Gaspari et al. [391] demonstrating a self-regulated jet capable of preserving the cool core (see also [382-386]). Collimated jet feedback is currently inadequately modeled in cosmological simulations; however, thermal blast feedback should not be dismissed as a potential mode operating at late times until it is confirmed that cored NCC underluminous groups do not exist. Meece et al. [392] found a hybrid kinetic jet with thermal heating in idealized hydro simulations could best achieve self-regulation and produce a cool core, whereas a thermal-only jet results in a cored profile that rapidly radiates energy away leading to a cooling catastrophe. Their kinetic-only model also achieves self-regulation, but appears too steady compared to observed AGN duty cycles.

The failure of cosmological simulations to reproduce the observed thermal structure of the IGrM (Section 4.1.2) may be related to cosmological simulation's inability to model narrow, high momentum flux, jet outflows. Narrow beams are better able to drill their way out through a highly pressurized IGrM/ICM that can easily stall an isotropic outflow, preventing the deposition of energy where it is needed. Nonetheless, narrow outflows have their own challenges, which become apparent through running idealized simulations. Firstly, as shown by Vernaleo and Reynolds [393] and confirmed by Cielo et al. [394], jets that fire in a fixed direction tend to deposit their energy at increasing larger distances and ultimately, end up doing so beyond the group/cluster core. As a result, such jets only delay the onset of catastrophic cool- 
ing, not prevent it. The second and equally vexing problem concerns the coupling between the narrow jets and the IGrM/ICM: how do narrow, bipolar jets manage to heat gas in the group/cluster cores in a near-isotropic fashion? This motivated Babul et al. [395] to argue for tilting jets, which change direction every so often as evidenced by observations detailed in this paper. Cielo et al. [394] - and most recently, Su et al. [396] - found that not only is tilting necessary, but the angle between jet events must also be reasonably large, and furthermore heated jets work better than cold jets. In effect, the desired outflows are those that have the appropriate energy/momentum flux, create near-spherical cocoons because these optimize energy transfer in transverse directions relatively to the jets, and in a time-averaged sense, distribute their energy in a near-isotropic fashion within the group/cluster core.

- X-ray detectability of new classes of groups: The largest number of diffuse object detections by eROSITA will be groups (e.g., [352]). The complete eRASS:8 survey should detect groups with $M_{500}>10^{13} \mathrm{M}_{\odot}$ out to $z=0.05$ [397]. While simulations of EAGLE and TNG100 eRASS:8 stacking show galactic-scale halos at $M_{500}<10^{13} \mathrm{M}_{\odot}$ will not be individually detected [361], eROSITA should observe groups in the local volume covered by CLoGS. Simulations will provide necessary guidance in the interpretation of any prospective cored NCC under-luminous groups and/or coalescing groups that have yet to virialize. It may well be that significantly under-luminous groups potentially exist as Pearson et al. [398] cannot detect with Chandra two of their 10 optically selected groups, which do not show signs of being unvirialized. The near future holds promise to detect new potential classes of groups-poor, under-luminous, and coalescing-in X-rays.

- Multi-phase gas stripping from group satellites: A shortcoming in common to all simulations that we have discussed in this review is the lack of a cold $\left(T \ll 10^{4} \mathrm{~K}\right)$ and dense molecular phase in the ISM of galaxies. Both observations (e.g., [399]) and idealized hydrodynamic simulations (e.g., [400]) clearly indicate that ram pressure has a different effect on the dense molecular phase from which stars are formed than the more tenuous, warmer components traced by $\mathrm{HI}$ and $\mathrm{H}$ II. Although post-processing can be used to estimate the molecular content of group satellites (albeit with strong assumptions; [307]), it cannot capture the different dynamical evolution of the two phases. Simulations with direct modeling of molecular gas-as is now often done in high-resolution zooms of individual galaxies (e.g., [113,299])—would therefore reveal a fundamentally new aspect of the interaction between the IGrM and satellite galaxies. Recent advances in subgrid cooling models [401] make such large-scale cold ISM simulations possible, but the high resolution required to resolve giant molecular clouds at least marginally $\left(\lesssim 10^{4} \mathrm{M}_{\odot}\right)$ makes them unfeasible on cluster scales for the foreseeable future. Galaxy groups, on the other hand, would be perfectly suited to exploring this additional facet of the baryon cycle in a full cosmological setting.

- The Sunyaev-Zel'dovich Effect: SZ stacking is already measuring the pressure and density profiles of groups from large radii inward. Cross-correlating large spectroscopic surveys (e.g., BOSS [402]) with high-resolution maps of the CMB from the Atacama Cosmology Telescope (ACT) has measured the extended pressure and density profiles of groups via the tSZ and kSZ effect respectively. Amodeo et al. [403] detected elevated gas pressure profiles outside $R_{200}$ of $z=0.55 M_{200}=10^{13.5} \mathrm{M}_{\odot}$ groups indicating that feedback energy equivalent to double the gaseous halo binding energy needs to coupled directly to the IGrM, which is significantly above predictions from TNG100 simulations and even more so the EAGLE simulations [140]. Schaan et al. [404] showed that $z=0.31 M_{200}=10^{13.7} \mathrm{M}_{\odot}$ groups are far more devoid of baryons in kSZ measurements than a Navarro et al. [405] (NFW) profile. Lim et al. [406] tested groups in Illustris, EAGLE, TNG300, and Magneticum simulations against Planck Collaboration et al. [407] stacks, finding that the $M_{500} \sim$ $10^{13.0-13.5} \mathrm{M}_{\odot}$ scale provides a very promising scale to constrain the nature of AGN feedback. The measurements of pressure, density, and, through division, temperature 
profiles of groups will dramatically increase in the 2020's as the Rubin Telescope comes on line and the Roman and Euclid Telescopes are launched, providing spectroscopic surveys to cross-correlate further CMB observations from the ACT, the Simons Observatory, the Large Millimeter Telescope, and CMB-S4. These future SZ surveys will provide standard calibrations against which simulated groups are compared.

\section{Final Statement}

Cosmological hydrodynamic simulations provide an aggregate picture of groups that is not yet available observationally. Some of the latest state-of-the-art simulation projects can reproduce key stellar observations of galaxies, while others are able to match essential gaseous properties of clusters and large scale structure. We approach the breakthrough when a single high-resolution cosmological simulation suite can match gaseous and stellar properties of both galaxies and clusters. The simulated groups in the intermediate range provide true testable predictions for upcoming observational datasets that include comprehensive galaxy surveys, all-sky $X$-ray maps, and deep radio surveys. Future observed group datasets synthesizing galaxy catalogues down to dwarf galaxies, $\mathrm{X}$-ray emission extending beyond $R_{500}$ and to masses below $M_{500}<10^{13.5} \mathrm{M}_{\odot}$, thermal and kinetic Sunyaev-Zel'dovich measurements, and UV absorption compilations plus 21-cm maps accounting for warm and cool gas component will provide creative new stress tests of the non-gravitational, baryonic physics in cosmological simulations.

Author Contributions: B.D.O.: lead author Sections 1, 2, 3.1-3.8, 4.1, 5.1, 5.3 and 6; A.B.: lead author Section 4.2, major contributions to Sections 4.1 and 5.3; Y.B.: lead author Section 4.4, major contributions to Sections 1-3 and 4.1; I.S.B.: lead author Sections 3.10 and 4.3; I.G.M.: lead author Sections 3.9, 4.5 and 5.2. All authors have read and agreed to the published version of the manuscript.

Funding: This research received no external funding.

Data Availability Statement: Much of the simulation data used here are publicly available, including for the EAGLE [408], IllustrisTNG [409], and SIMBA (http:/ / simba.roe.ac.uk/ (accessed on 10 June 2021)) datasets. Figures uniquely generated for this review by the authors include Figures 3-5, 6 (left panel), and Figures 12-18.

Acknowledgments: We are grateful to the three anonymous referees who provided thorough reports that substantially improved the scope and clarity of this review. We thank the following astrophysicists for thought-provoking conversations, observational and simulation data and figures, and essential guidance in the production of this review: Sarah Appleby, Michelle Cluver, Weiguang Cui, Romeel Davé, Dominique Eckert, Seoyoung Lyla Jung, Amandine Le Brun, Ilani Loubser, Lorenzo Lovisari, Daisuke Nagai, Ewan O'Sullivan, Douglas Rennehan, Vida Saeedzadeh, Zhiwei Shao, Prateek Sharma, Aurora Simionescu, Ming Sun, Michael Tremmel, Nastasha Wijers, and Mark Voit. This research was supported in part by the KITP National Science Foundation under Grant No. NSF PHY-1748958, and by the Netherlands Organization for Scientific Research (NWO) through Veni grant number 639.041.751. This review is reliant on the public data release and accessibility generously provided by the EAGLE, IllustrisTNG, and SIMBA teams. A number of calculations and figures presented in this review were done using (i) high performance computing facilities at Liverpool John Moores University, partly funded by the Royal Society and LJMU's Faculty of Engineering and Technology, and (ii) advanced research computing resources provided by Compute/Calcul Canada. I.G.M. acknowledges support by the European Research Council (ERC) under the European Union's Horizon 2020 research and innovation programme (grant agreement No. 769130). A.B. acknowledges research support from Natural Sciences and Engineering Research Council of Canada (NSERC) and Compute Canada.

Conflicts of Interest: The authors declare no conflict of interest.

\section{Notes}

1 In the remainder of this review, we omit the ' $c$ ' suffix that identifies the overdensity as measured with respect to the critical, rather than for example, mean, density of the universe

2 All conversions are median differences obtained from the IllustrisTNG300 simulation 
We note that the ability of particles to move through the simulation volume is not particular to this approach, and is also an integral feature of the SPH approach

\section{References}

1. Lovisari, L.; Ettori, S.; Gaspari, M.; Giles, P.A. Scaling Properties of Galaxy Groups. Universe 2021, 7, 139. [CrossRef]

2. Sun, M.; Voit, G.M.; Donahue, M.; Jones, C.; Forman, W.; Vikhlinin, A. Chandra Studies of the X-Ray Gas Properties of Galaxy Groups. Astrophys. J. 2009, 693, 1142-1172. [CrossRef]

3. Eckmiller, H.J.; Hudson, D.S.; Reiprich, T.H. Testing the low-mass end of X-ray scaling relations with a sample of Chandra galaxy groups. Astron. Astrophys. 2011, 535, A105. [CrossRef]

4. Bharadwaj, V.; Reiprich, T.H.; Lovisari, L.; Eckmiller, H.J. Extending the $\mathrm{L}_{X}-\mathrm{T}$ relation from clusters to groups. Impact of cool core nature, AGN feedback, and selection effects. Astron. Astrophys. 2015, 573, A75. [CrossRef]

5. Lovisari, L.; Reiprich, T.H.; Schellenberger, G. Scaling properties of a complete X-ray selected galaxy group sample. Astron. Astrophys. 2015, 573, A118. [CrossRef]

6. Mitchell, N.L.; McCarthy, I.G.; Bower, R.G.; Theuns, T.; Crain, R.A. On the origin of cores in simulated galaxy clusters. Mon. Not. R. Astron. Soc. 2009, 395, 180-196. [CrossRef]

7. Le Brun, A.M.C.; McCarthy, I.G.; Schaye, J.; Ponman, T.J. Towards a realistic population of simulated galaxy groups and clusters. Mon. Not. R. Astron. Soc. 2014, 441, 1270-1290. [CrossRef]

8. O'Sullivan, E.; Ponman, T.J.; Kolokythas, K.; Raychaudhury, S.; Babul, A.; Vrtilek, J.M.; David, L.P.; Giacintucci, S.; Gitti, M.; Haines, C.P. The Complete Local Volume Groups Sample-I. Sample selection and X-ray properties of the high-richness subsample. Mon. Not. R. Astron. Soc. 2017, 472, 1482-1505. [CrossRef]

9. Cavagnolo, K.W.; Donahue, M.; Voit, G.M.; Sun, M. Intracluster Medium Entropy Profiles for a Chandra Archival Sample of Galaxy Clusters. Astrophys. J. Suppl. 2009, 182, 12-32. [CrossRef]

10. Pratt, G.W.; Croston, J.H.; Arnaud, M.; Böhringer, H. Galaxy cluster X-ray luminosity scaling relations from a representative local sample (REXCESS). Astron. Astrophys. 2009, 498, 361-378. [CrossRef]

11. Wetzel, A.R.; Tinker, J.L.; Conroy, C. Galaxy evolution in groups and clusters: Star formation rates, red sequence fractions and the persistent bimodality. Mon. Not. R. Astron. Soc. 2012, 424, 232-243. [CrossRef]

12. Davies, L.J.M.; Robotham, A.S.G.; Lagos, C.d.P.; Driver, S.P.; Stevens, A.R.H.; Bahé, Y.M.; Alpaslan, M.; Bremer, M.N.; Brown, M.J.I.; Brough, S.; et al. Galaxy and Mass Assembly (GAMA): Environmental quenching of centrals and satellites in groups. Mon. Not. R. Astron. Soc. 2019, 483, 5444-5458. [CrossRef] 
13. Moffett, A.J.; Ingarfield, S.A.; Driver, S.P.; Robotham, A.S.G.; Kelvin, L.S.; Lange, R.; Meštrić, U.; Alpaslan, M.; Baldry, I.K.; Bland-Hawthorn, J.; et al. Galaxy And Mass Assembly (GAMA): The stellar mass budget by galaxy type. Mon. Not. R. Astron. Soc. 2016, 457, 1308-1319. [CrossRef]

14. Somerville, R.S.; Davé, R. Physical Models of Galaxy Formation in a Cosmological Framework. Annu. Rev. Astron. Astrophys. 2015, 53, 51-113. [CrossRef]

15. Tumlinson, J.; Peeples, M.S.; Werk, J.K. The Circumgalactic Medium. Annu. Rev. Astron. Astrophys. 2017, 55, 389-432. [CrossRef]

16. Voit, G.M.; Donahue, M.; Bryan, G.L.; McDonald, M. Regulation of star formation in giant galaxies by precipitation, feedback and conduction. Nature 2015, 519, 203-206. [CrossRef]

17. Gaspari, M.; Temi, P.; Brighenti, F. Raining on black holes and massive galaxies: The top-down multiphase condensation model. Mon. Not. R. Astron. Soc. 2017, 466, 677-704. [CrossRef]

18. Emerick, A.; Bryan, G.; Putman, M.E. Warm gas in and around simulated galaxy clusters as probed by absorption lines. Mon. Not. R. Astron. Soc. 2015, 453, 4051-4069. [CrossRef]

19. Butsky, I.S.; Burchett, J.N.; Nagai, D.; Tremmel, M.; Quinn, T.R.; Werk, J.K. Ultraviolet signatures of the multiphase intracluster and circumgalactic media in the ROMULUSC simulation. Mon. Not. R. Astron. Soc. 2019, 490, 4292-4306. [CrossRef]

20. McDonald, M.; Veilleux, S.; Mushotzky, R. The Effect of Environment on the Formation of H $\alpha$ Filaments and Cool Cores in Galaxy Groups and Clusters. Astrophys. J. 2011, 731, 33. [CrossRef]

21. Tremblay, G.R.; Combes, F.; Oonk, J.B.R.; Russell, H.R.; McDonald, M.A.; Gaspari, M.; Husemann, B.; Nulsen, P.E.J.; McNamara, B.R.; Hamer, S.L.; et al. A Galaxy-scale Fountain of Cold Molecular Gas Pumped by a Black Hole. Astrophys. J. 2018, 865, 13. [CrossRef]

22. Tonnesen, S.; Bryan, G.L.; Chen, R. How to Light it Up: Simulating Ram-pressure Stripped X-ray Bright Tails. Astrophys. J. 2011, 731, 98. [CrossRef]

23. Yun, K.; Pillepich, A.; Zinger, E.; Nelson, D.; Donnari, M.; Joshi, G.; Rodriguez-Gomez, V.; Genel, S.; Weinberger, R.; Vogelsberger, M.; et al. Jellyfish galaxies with the IllustrisTNG simulations-I. Gas-stripping phenomena in the full cosmological context. Mon. Not. R. Astron. Soc. 2019, 483, 1042-1066. [CrossRef]

24. Campitiello, M.G.; Ignesti, A.; Gitti, M.; Brighenti, F.; Radovich, M.; Wolter, A.; Tomičić, N.; Bellhouse, C.; Poggianti, B.M.; Moretti, A.; et al. GASP XXXIV: Unfolding the Thermal Side of Ram Pressure Stripping in the Jellyfish Galaxy JO201. Astrophys. J. 2021, 911, 144. [CrossRef]

25. McGee, S.L.; Bower, R.G.; Balogh, M.L. Overconsumption, outflows and the quenching of satellite galaxies. Mon. Not. R. Astron. Soc. 2014, 442, L105-L109. [CrossRef]

26. Burchett, J.N.; Tripp, T.M.; Wang, Q.D.; Willmer, C.N.A.; Bowen, D.V.; Jenkins, E.B. Warm-hot gas in X-ray bright galaxy clusters and the H I-deficient circumgalactic medium in dense environments. Mon. Not. R. Astron. Soc. 2018, 475, 2067-2085. [CrossRef]

27. Stocke, J.T.; Keeney, B.A.; Danforth, C.W.; Oppenheimer, B.D.; Pratt, C.T.; Berlind, A.A.; Impey, C.; Jannuzi, B. The Ultraviolet Detection of Diffuse Gas in Galaxy Groups. Astrophys. J. Suppl. 2019, 240, 15. [CrossRef]

28. Borthakur, S.; Yun, M.S.; Verdes-Montenegro, L. Detection of Diffuse Neutral Intragroup Medium in Hickson Compact Groups. Astrophys. J. 2010, 710, 385-407. [CrossRef]

29. Oman, K.A.; Bahé, Y.M.; Healy, J.; Hess, K.M.; Hudson, M.J.; Verheijen, M.A.W. A homogeneous measurement of the delay between the onsets of gas stripping and star formation quenching in satellite galaxies of groups and clusters. Mon. Not. R. Astron. Soc. 2021, 501, 5073-5095. [CrossRef]

30. Brown, T.; Catinella, B.; Cortese, L.; Lagos, C.d.P.; Davé, R.; Kilborn, V.; Haynes, M.P.; Giovanelli, R.; Rafieferantsoa, M. Cold gas stripping in satellite galaxies: From pairs to clusters. Mon. Not. R. Astron. Soc. 2017, 466, 1275-1289. [CrossRef]

31. Bahé, Y.M.; Schaye, J.; Barnes, D.J.; Dalla Vecchia, C.; Kay, S.T.; Bower, R.G.; Hoekstra, H.; McGee, S.L.; Theuns, T. Disruption of satellite galaxies in simulated groups and clusters: The roles of accretion time, baryons, and pre-processing. Mon. Not. R. Astron. Soc. 2019, 485, 2287-2311. [CrossRef]

32. Fujita, Y. Pre-Processing of Galaxies before Entering a Cluster. Publ. Astron. Soc. Jpn. 2004, 56, 29-43. [CrossRef]

33. Wetzel, A.R.; Tinker, J.L.; Conroy, C.; van den Bosch, F.C. Galaxy evolution in groups and clusters: Satellite star formation histories and quenching time-scales in a hierarchical Universe. Mon. Not. R. Astron. Soc. 2013, 432, 336-358. [CrossRef]

34. Donnari, M.; Pillepich, A.; Joshi, G.D.; Nelson, D.; Genel, S.; Marinacci, F.; Rodriguez-Gomez, V.; Pakmor, R.; Torrey, P.; Vogelsberger, M.; et al. Quenched fractions in the IllustrisTNG simulations: The roles of AGN feedback, environment, and pre-processing. Mon. Not. R. Astron. Soc. 2021, 500, 4004-4024. [CrossRef]

35. Jenkins, A.; Frenk, C.S.; White, S.D.M.; Colberg, J.M.; Cole, S.; Evrard, A.E.; Couchman, H.M.P.; Yoshida, N. The mass function of dark matter haloes. Mon. Not. R. Astron. Soc. 2001, 321, 372-384. [CrossRef]

36. Bryan, G.L.; Norman, M.L. Statistical Properties of X-ray Clusters: Analytic and Numerical Comparisons. Astrophys. J. 1998, 495, 80-99. [CrossRef]

37. Liang, L.; Durier, F.; Babul, A.; Davé, R.; Oppenheimer, B.D.; Katz, N.; Fardal, M.; Quinn, T. The growth and enrichment of intragroup gas. Mon. Not. R. Astron. Soc. 2016, 456, 4266-4290. [CrossRef]

38. Vogelsberger, M.; Genel, S.; Springel, V.; Torrey, P.; Sijacki, D.; Xu, D.; Snyder, G.; Nelson, D.; Hernquist, L. Introducing the Illustris Project: Simulating the coevolution of dark and visible matter in the Universe. Mon. Not. R. Astron. Soc. 2014, 444, 1518-1547. [CrossRef] 
39. Schaye, J.; Crain, R.A.; Bower, R.G.; Furlong, M.; Schaller, M.; Theuns, T.; Dalla Vecchia, C.; Frenk, C.S.; McCarthy, I.G.; Helly, J.C.; et al. The EAGLE project: Simulating the evolution and assembly of galaxies and their environments. Mon. Not. R. Astron. Soc. 2015, 446, 521-554.

[CrossRef]

40. Dubois, Y.; Peirani, S.; Pichon, C.; Devriendt, J.; Gavazzi, R.; Welker, C.; Volonteri, M. The HORIZON-AGN simulation: Morphological diversity of galaxies promoted by AGN feedback. Mon. Not. R. Astron. Soc. 2016, 463, 3948-3964. [CrossRef]

41. McCarthy, I.G.; Schaye, J.; Bird, S.; Le Brun, A.M.C. The BAHAMAS project: Calibrated hydrodynamical simulations for large-scale structure cosmology. Mon. Not. R. Astron. Soc. 2017, 465, 2936-2965. [CrossRef]

42. Bahé, Y.M.; Barnes, D.J.; Dalla Vecchia, C.; Kay, S.T.; White, S.D.M.; McCarthy, I.G.; Schaye, J.; Bower, R.G.; Crain, R.A.; Theuns, T.; et al. The Hydrangea simulations: Galaxy formation in and around massive clusters. Mon. Not. R. Astron. Soc. 2017, 470, 4186-4208. [CrossRef]

43. Barnes, D.J.; Kay, S.T.; Bahé, Y.M.; Dalla Vecchia, C.; McCarthy, I.G.; Schaye, J.; Bower, R.G.; Jenkins, A.; Thomas, P.A.; Schaller, M.; et al. The Cluster-EAGLE project: Global properties of simulated clusters with resolved galaxies. Mon. Not. R. Astron. Soc. 2017, 471, 1088-1106. [CrossRef]

44. Henden, N.A.; Puchwein, E.; Shen, S.; Sijacki, D. The FABLE simulations: A feedback model for galaxies, groups, and clusters. Mon. Not. R. Astron. Soc. 2018, 479, 5385-5412. [CrossRef]

45. Cui, W.; Knebe, A.; Yepes, G.; Pearce, F.; Power, C.; Dave, R.; Arth, A.; Borgani, S.; Dolag, K.; Elahi, P.; et al. The Three Hundred project: A large catalogue of theoretically modelled galaxy clusters for cosmological and astrophysical applications. Mon. Not. R. Astron. Soc. 2018, 480, 2898-2915. [CrossRef]

46. Pillepich, A.; Springel, V.; Nelson, D.; Genel, S.; Naiman, J.; Pakmor, R.; Hernquist, L.; Torrey, P.; Vogelsberger, M.; Weinberger, R.; et al. Simulating galaxy formation with the IllustrisTNG model. Mon. Not. R. Astron. Soc. 2018, 473, 4077-4106. [CrossRef]

47. Nelson, D.; Pillepich, A.; Springel, V.; Weinberger, R.; Hernquist, L.; Pakmor, R.; Genel, S.; Torrey, P.; Vogelsberger, M.; Kauffmann, G.; Marinacci, F.; Naiman, J. First results from the IllustrisTNG simulations: The galaxy colour bimodality. Mon. Not. R. Astron. Soc. 2018, 475, 624-647. [CrossRef]

48. Tremmel, M.; Karcher, M.; Governato, F.; Volonteri, M.; Quinn, T.R.; Pontzen, A.; Anderson, L.; Bellovary, J. The Romulus cosmological simulations: A physical approach to the formation, dynamics and accretion models of SMBHs. Mon. Not. R. Astron. Soc. 2017, 470, 1121-1139. [CrossRef]

49. Tremmel, M.; Quinn, T.R.; Ricarte, A.; Babul, A.; Chadayammuri, U.; Natarajan, P.; Nagai, D.; Pontzen, A.; Volonteri, M. Introducing ROMULUSC: A cosmological simulation of a galaxy cluster with an unprecedented resolution. Mon. Not. R. Astron. Soc. 2019, 483, 3336-3362. [CrossRef]

50. Davé, R.; Anglés-Alcázar, D.; Narayanan, D.; Li, Q.; Rafieferantsoa, M.H.; Appleby, S. SIMBA: Cosmological simulations with black hole growth and feedback. Mon. Not. R. Astron. Soc. 2019, 486, 2827-2849. [CrossRef]

51. Nelson, D.; Pillepich, A.; Springel, V.; Pakmor, R.; Weinberger, R.; Genel, S.; Torrey, P.; Vogelsberger, M.; Marinacci, F.; Hernquist L. First results from the TNG50 simulation: Galactic outflows driven by supernovae and black hole feedback. Mon. Not. R. Astron. Soc. 2019, 490, 3234-3261. [CrossRef]

52. Teyssier, R. Grid-Based Hydrodynamics in Astrophysical Fluid Flows. Annu. Rev. Astron. Astrophys. 2015, 53, 325-364. [CrossRef]

53. Springel, V. Smoothed Particle Hydrodynamics in Astrophysics. Annu. Rev. Astron. Astrophys. 2010, 48, 391-430. [CrossRef]

54. Price, D.J. Smoothed particle hydrodynamics and magnetohydrodynamics. J. Comput. Phys. 2012, 231, 759-794. [CrossRef]

55. Springel, V. E pur si muove: Galilean-invariant cosmological hydrodynamical simulations on a moving mesh. Mon. Not. R. Astron. Soc. 2010, 401, 791-851. [CrossRef]

56. Hopkins, P.F. A new class of accurate, mesh-free hydrodynamic simulation methods. Mon. Not. R. Astron. Soc. 2015, 450, 53-110. [CrossRef]

57. Koshizuka, S.; Oka, Y. Moving-particle semi-implicit method for fragmentation of incompressible fluid. Nucl. Sci. Eng. 1996, 123, 421-434. [CrossRef]

58. Hu, C.Y.; Naab, T.; Walch, S.; Moster, B.P.; Oser, L. SPHGal: Smoothed particle hydrodynamics with improved accuracy for galaxy simulations. Mon. Not. R. Astron. Soc. 2014, 443, 1173-1191. [CrossRef]

59. Schaller, M.; Dalla Vecchia, C.; Schaye, J.; Bower, R.G.; Theuns, T.; Crain, R.A.; Furlong, M.; McCarthy, I.G. The EAGLE simulations of galaxy formation: The importance of the hydrodynamics scheme. Mon. Not. R. Astron. Soc. 2015, 454, $2277-2291$. [CrossRef]

60. Beck, A.M.; Murante, G.; Arth, A.; Remus, R.S.; Teklu, A.F.; Donnert, J.M.F.; Planelles, S.; Beck, M.C.; Förster, P.; Imgrund, M.; et al. An improved SPH scheme for cosmological simulations. Mon. Not. R. Astron. Soc. 2016, 455, 2110-2130. [CrossRef]

61. Borrow, J.; Schaller, M.; Bower, R.G.; Schaye, J. Sphenix: Smoothed Particle Hydrodynamics for the next generation of galaxy formation simulations. arXiv 2020, arXiv:2012.03974.

62. Crain, R.A.; Schaye, J.; Bower, R.G.; Furlong, M.; Schaller, M.; Theuns, T.; Dalla Vecchia, C.; Frenk, C.S.; McCarthy, I.G. The EAGLE simulations of galaxy formation: Calibration of subgrid physics and model variations. Mon. Not. R. Astron. Soc. 2015, 450, 1937-1961. [CrossRef]

63. McAlpine, S.; Helly, J.C.; Schaller, M.; Trayford, J.W.; Qu, Y.; Furlong, M.; Bower, R.G.; Crain, R.A.; Schaye, J.; Theuns, T.; et al. The EAGLE simulations of galaxy formation: Public release of halo and galaxy catalogues. Astron. Comput. 2016, 15, 72-89. [CrossRef] 
64. Marinacci, F.; Vogelsberger, M.; Pakmor, R.; Torrey, P.; Springel, V.; Hernquist, L.; Nelson, D.; Weinberger, R.; Pillepich, A.; Naiman, J.; et al. First results from the IllustrisTNG simulations: Radio haloes and magnetic fields. Mon. Not. R. Astron. Soc. 2018, 480, 5113-5139. [CrossRef]

65. Naiman, J.P.; Pillepich, A.; Springel, V.; Ramirez-Ruiz, E.; Torrey, P.; Vogelsberger, M.; Pakmor, R.; Nelson, D.; Marinacci, F.; Hernquist, L.; et al. First results from the IllustrisTNG simulations: A tale of two elements-Chemical evolution of magnesium and europium. Mon. Not. R. Astron. Soc. 2018, 477, 1206-1224. [CrossRef]

66. Springel, V.; Pakmor, R.; Pillepich, A.; Weinberger, R.; Nelson, D.; Hernquist, L.; Vogelsberger, M.; Genel, S.; Torrey, P.; Marinacci, F.; et al. First results from the IllustrisTNG simulations: Matter and galaxy clustering. Mon. Not. R. Astron. Soc. 2018, 475, 676-698. [CrossRef]

67. Pillepich, A.; Nelson, D.; Hernquist, L.; Springel, V.; Pakmor, R.; Torrey, P.; Weinberger, R.; Genel, S.; Naiman, J.P.; Marinacci, F.; et al. First results from the IllustrisTNG simulations: The stellar mass content of groups and clusters of galaxies. Mon. Not. R. Astron. Soc. 2018, 475, 648-675. [CrossRef]

68. Donnari, M.; Pillepich, A.; Nelson, D.; Marinacci, F.; Vogelsberger, M.; Hernquist, L. Quenched fractions in the IllustrisTNG simulations: Comparison with observations and other theoretical models. arXiv 2020, arXiv:2008.00004.

69. Dolag, K.; Komatsu, E.; Sunyaev, R. SZ effects in the Magneticum Pathfinder simulation: Comparison with the Planck, SPT, and ACT results. Mon. Not. R. Astron. Soc. 2016, 463, 1797-1811. [CrossRef]

70. Castro, T.; Borgani, S.; Dolag, K.; Marra, V.; Quartin, M.; Saro, A.; Sefusatti, E. On the impact of baryons on the halo mass function, bias, and cluster cosmology. Mon. Not. R. Astron. Soc. 2021, 500, 2316-2335.

[CrossRef]

71. Ragagnin, A.; Dolag, K.; Moscardini, L.; Biviano, A.; D’Onofrio, M. Dependency of halo concentration on mass, redshift and fossilness in Magneticum hydrodynamic simulations. Mon. Not. R. Astron. Soc. 2019, 486, 4001-4012. [CrossRef]

72. Chadayammuri, U.; Tremmel, M.; Nagai, D.; Babul, A.; Quinn, T. Fountains and storms: The effects of AGN feedback and mergers on the evolution of the intracluster medium in the ROMULUSC simulation. Mon. Not. R. Astron. Soc. 2021, 504, $3922-3937$. [CrossRef]

73. Jung, S.L.; Rennehan, D.; Saeedzadeh, V.; Babul, A.; Tremmel, M.; Quinn, T.R.; S.I., L.; Sukyoung, K.Y. Brightest Group Galaxies in Romulus Simulations: A link between kinematics and morphology. In preparation.

74. Pillepich, A.; Nelson, D.; Springel, V.; Pakmor, R.; Torrey, P.; Weinberger, R.; Vogelsberger, M.; Marinacci, F.; Genel, S.; van der Wel, A.; et al. First results from the TNG50 simulation: The evolution of stellar and gaseous discs across cosmic time. Mon. Not. R. Astron. Soc. 2019, 490, 3196-3233. [CrossRef]

75. Feldmann, R.; Carollo, C.M.; Mayer, L.; Renzini, A.; Lake, G.; Quinn, T.; Stinson, G.S.; Yepes, G. The Evolution of Central Group Galaxies in Hydrodynamical Simulations. Astrophys. J. 2010, 709, 218-240. [CrossRef]

76. Oppenheimer, B.D.; Crain, R.A.; Schaye, J.; Rahmati, A.; Richings, A.J.; Trayford, J.W.; Tumlinson, J.; Bower, R.G.; Schaller, M.; Theuns, T. Bimodality of low-redshift circumgalactic O VI in non-equilibrium EAGLE zoom simulations. Mon. Not. R. Astron. Soc. 2016, 460, 2157-2179. [CrossRef]

77. Joshi, G.D.; Parker, L.C.; Wadsley, J.; Keller, B.W. The trajectories of galaxies in groups: Mass-loss and preprocessing. Mon. Not. R. Astron. Soc. 2019, 483, 235-248. [CrossRef]

78. Eckert, D.; Gaspari, M.; Gastaldello, F.; Le Brun, A.M.C.; O'Sullivan, E. Feedback from Active Galactic Nuclei in Galaxy Groups. Universe 2021, 7, 142. [CrossRef]

79. Dolag, K.; Stasyszyn, F. An MHD GADGET for cosmological simulations. Mon. Not. R. Astron. Soc. 2009, 398, 1678-1697. [CrossRef]

80. Pakmor, R.; Springel, V. Simulations of magnetic fields in isolated disc galaxies. Mon. Not. R. Astron. Soc. 2013, 432, 176-193. [CrossRef]

81. Frenk, C.S.; White, S.D.M.; Bode, P.; Bond, J.R.; Bryan, G.L.; Cen, R.; Couchman, H.M.P.; Evrard, A.E.; Gnedin, N.; Jenkins, A.; et al. The Santa Barbara Cluster Comparison Project: A Comparison of Cosmological Hydrodynamics Solutions. Astrophys. J. 1999, 525, 554-582. [CrossRef]

82. Wadsley, J.W.; Veeravalli, G.; Couchman, H.M.P. On the treatment of entropy mixing in numerical cosmology. Mon. Not. R. Astron. Soc. 2008, 387, 427-438. [CrossRef]

83. Rasia, E.; Borgani, S.; Murante, G.; Planelles, S.; Beck, A.M.; Biffi, V.; Ragone-Figueroa, C.; Granato, G.L.; Steinborn, L.K.; Dolag, K. Cool Core Clusters from Cosmological Simulations. Astrophys. J. Lett. 2015, 813, L17. [CrossRef]

84. Hahn, O.; Martizzi, D.; Wu, H.Y.; Evrard, A.E.; Teyssier, R.; Wechsler, R.H. rhapsody-g simulations-I. The cool cores, hot gas and stellar content of massive galaxy clusters. Mon. Not. R. Astron. Soc. 2017, 470, 166-186. [CrossRef]

85. Sembolini, F.; Yepes, G.; Pearce, F.R.; Knebe, A.; Kay, S.T.; Power, C.; Cui, W.; Beck, A.M.; Borgani, S.; Dalla Vecchia, C.; et al. nIFTy galaxy cluster simulations-I. Dark matter and non-radiative models. Mon. Not. R. Astron. Soc. 2016, 457, 4063-4080. [CrossRef]

86. Haardt, F.; Madau, P. Radiative Transfer in a Clumpy Universe. IV. New Synthesis Models of the Cosmic UV/X-Ray Background. Astrophys. J. 2012, 746, 125. [CrossRef]

87. Ferland, G.J.; Porter, R.L.; van Hoof, P.A.M.; Williams, R.J.R.; Abel, N.P.; Lykins, M.L.; Shaw, G.; Henney, W.J.; Stancil, P.C. The 2013 Release of Cloudy. Rev. Mex. Astron. Astrofis. 2013, 49, 137-163. 
88. Wiersma, R.P.C.; Schaye, J.; Smith, B.D. The effect of photoionization on the cooling rates of enriched, astrophysical plasmas. Mon. Not. R. Astron. Soc. 2009, 393, 99-107. [CrossRef]

89. Smith, B.D.; Bryan, G.L.; Glover, S.C.O.; Goldbaum, N.J.; Turk, M.J.; Regan, J.; Wise, J.H.; Schive, H.Y.; Abel, T.; Emerick, A.; et al. GRACKLE: A chemistry and cooling library for astrophysics. Mon. Not. R. Astron. Soc. 2017, 466, 2217-2234. [CrossRef]

90. Vogelsberger, M.; Genel, S.; Sijacki, D.; Torrey, P.; Springel, V.; Hernquist, L. A model for cosmological simulations of galaxy formation physics. Mon. Not. R. Astron. Soc. 2013, 436, 3031-3067. [CrossRef]

91. Cen, R.; Fang, T. Where Are the Baryons? III. Nonequilibrium Effects and Observables. Astrophys. J. 2006, 650, 573-591. [CrossRef]

92. Gnat, O.; Sternberg, A. Time-dependent Ionization in Radiatively Cooling Gas. Astrophys. J. Suppl. 2007, 168, 213-230. [CrossRef]

93. Gnat, O.; Ferland, G.J. Ion-by-ion Cooling Efficiencies. Astrophys. J. Suppl. 2012, 199, 20. [CrossRef]

94. Oppenheimer, B.D.; Schaye, J. Non-equilibirum ionization and cooling of metal-enriched gas in the presence of a photoionization background. Mon. Not. R. Astron. Soc. 2013, 434, 1043-1062. [CrossRef]

95. Lewis, G.F.; Babul, A.; Katz, N.; Quinn, T.; Hernquist, L.; Weinberg, D.H. The effects of gasdynamics, cooling, star formation, and numerical resolution in simulations of cluster formation. Astrophys. J. 2000, 536, 623. [CrossRef]

96. Voit, G.M.; Kay, S.T.; Bryan, G.L. The baseline intracluster entropy profile from gravitational structure formation. Mon. Not. R. Astron. Soc. 2005, 364, 909-916. [CrossRef]

97. Taylor, J.E.; Babul, A. The evolution of substructure in galaxy, group and cluster haloes - I. Basic dynamics. Mon. Not. R. Astron. Soc. 2004, 348, 811-830. [CrossRef]

98. Barnes, E.I.; Williams, L.L.R.; Babul, A.; Dalcanton, J.J. Density Profiles of Collisionless Equilibria. II. Anisotropic Spherical Systems. Astrophys. J. 2007, 654, 814-824. [CrossRef]

99. Springel, V.; Hernquist, L. Cosmological smoothed particle hydrodynamics simulations: A hybrid multiphase model for star formation. Mon. Not. R. Astron. Soc. 2003, 339, 289-311. [CrossRef]

100. Schaye, J.; Dalla Vecchia, C. On the relation between the Schmidt and Kennicutt-Schmidt star formation laws and its implications for numerical simulations. Mon. Not. R. Astron. Soc. 2008, 383, 1210-1222. [CrossRef]

101. Davé, R.; Thompson, R.; Hopkins, P.F. MUFASA: Galaxy formation simulations with meshless hydrodynamics. Mon. Not. R. Astron. Soc. 2016, 462, 3265-3284. [CrossRef]

102. Kennicutt, J.; Robert, C. Star Formation in Galaxies Along the Hubble Sequence. Annu. Rev. Astron. Astrophys. 1998, 36, 189-232. [CrossRef]

103. Kroupa, P. On the variation of the initial mass function. Mon. Not. R. Astron. Soc. 2001, 322, 231-246. [CrossRef]

104. Chabrier, G. Galactic Stellar and Substellar Initial Mass Function. Publ. Astron. Soc. Pac. 2003, 115, 763-795. [CrossRef]

105. Gastaldello, F.; Simionescu, A.; Mernier, F.; Biffi, V.; Gaspari, M.; Sato, K.; Matsushita, K. The metal content of the hot atmospheres of galaxy groups. Universe, accepted.

106. Wiersma, R.P.C.; Schaye, J.; Theuns, T.; Dalla Vecchia, C.; Tornatore, L. Chemical enrichment in cosmological, smoothed particle hydrodynamics simulations. Mon. Not. R. Astron. Soc. 2009, 399, 574-600. [CrossRef]

107. Nelson, D.; Kauffmann, G.; Pillepich, A.; Genel, S.; Springel, V.; Pakmor, R.; Hernquist, L.; Weinberger, R.; Torrey, P.; Vogelsberger, M.; Marinacci, F. The abundance, distribution, and physical nature of highly ionized oxygen O VI, O VII, and O VIII in IllustrisTNG. Mon. Not. R. Astron. Soc. 2018, 477, 450-479. [CrossRef]

108. Tornatore, L.; Borgani, S.; Dolag, K.; Matteucci, F. Chemical enrichment of galaxy clusters from hydrodynamical simulations. Mon. Not. R. Astron. Soc. 2007, 382, 1050-1072. [CrossRef]

109. Davé, R.; Oppenheimer, B.D.; Sivanand am, S. Enrichment and pre-heating in intragroup gas from galactic outflows. Mon. Not. R. Astron. Soc. 2008, 391, 110-123. [CrossRef]

110. Peterson, J.R.; Kahn, S.M.; Paerels, F.B.S.; Kaastra, J.S.; Tamura, T.; Bleeker, J.A.M.; Ferrigno, C.; Jernigan, J.G. High-Resolution X-Ray Spectroscopic Constraints on Cooling-Flow Models for Clusters of Galaxies. Astrophys. J. 2003, 590, 207-224. [CrossRef]

111. Biffi, V.; Mernier, F.; Medvedev, P. Enrichment of the Hot Intracluster Medium: Numerical Simulations. Space Sci. Rev. 2018, 214, 123. [CrossRef]

112. Shen, S.; Wadsley, J.; Stinson, G. The enrichment of the intergalactic medium with adiabatic feedback - I. Metal cooling and metal diffusion. Mon. Not. R. Astron. Soc. 2010, 407, 1581-1596. [CrossRef]

113. Hopkins, P.F.; Wetzel, A.; Kereš, D.; Faucher-Giguère, C.A.; Quataert, E.; Boylan-Kolchin, M.; Murray, N.; Hayward, C.C.; Garrison-Kimmel, S.; Hummels, C.; et al. FIRE-2 simulations: Physics versus numerics in galaxy formation. Mon. Not. R. Astron. Soc. 2018, 480, 800-863. [CrossRef]

114. Rennehan, D. Mixing matters. arXiv 2021, arXiv:2104.07673.

115. Oppenheimer, B.D.; Davé, R.; Kereš, D.; Fardal, M.; Katz, N.; Kollmeier, J.A.; Weinberg, D.H. Feedback and recycled wind accretion: Assembling the $\mathrm{z}=0$ galaxy mass function. Mon. Not. R. Astron. Soc. 2010, 406, 2325-2338. [CrossRef]

116. Schaye, J.; Dalla Vecchia, C.; Booth, C.M.; Wiersma, R.P.C.; Theuns, T.; Haas, M.R.; Bertone, S.; Duffy, A.R.; McCarthy, I.G.; van de Voort, F. The physics driving the cosmic star formation history. Mon. Not. R. Astron. Soc. 2010, 402, 1536-1560. [CrossRef]

117. Scannapieco, C.; Wadepuhl, M.; Parry, O.H.; Navarro, J.F.; Jenkins, A.; Springel, V.; Teyssier, R.; Carlson, E.; Couchman, H.M.P.; Crain, R.A.; et al. The Aquila comparison project: The effects of feedback and numerical methods on simulations of galaxy formation. Mon. Not. R. Astron. Soc. 2012, 423, 1726-1749. [CrossRef] 
118. Haas, M.R.; Schaye, J.; Booth, C.M.; Dalla Vecchia, C.; Springel, V.; Theuns, T.; Wiersma, R.P.C. Physical properties of simulated galaxy populations at $\mathrm{z}=2-\mathrm{I}$. Effect of metal-line cooling and feedback from star formation and AGN. Mon. Not. R. Astron. Soc. 2013, 435, 2931-2954. [CrossRef]

119. Torrey, P.; Vogelsberger, M.; Genel, S.; Sijacki, D.; Springel, V.; Hernquist, L. A model for cosmological simulations of galaxy formation physics: multi-epoch validation. Mon. Not. R. Astron. Soc. 2014, 438, 1985-2004. [CrossRef]

120. White, S.D.M.; Frenk, C.S. Galaxy Formation through Hierarchical Clustering. Astrophys. J. 1991, 379, 52. [CrossRef]

121. Oppenheimer, B.D.; Davé, R.; Finlator, K. Tracing the re-ionization-epoch intergalactic medium with metal absorption lines. Mon. Not. R. Astron. Soc. 2009, 396, 729-758. [CrossRef]

122. Keating, L.C.; Puchwein, E.; Haehnelt, M.G.; Bird, S.; Bolton, J.S. Testing the effect of galactic feedback on the IGM at $\mathrm{z} \sim 6 \mathrm{with}$ metal-line absorbers. Mon. Not. R. Astron. Soc. 2016, 461, 606-626. [CrossRef]

123. Finlator, K.; Oppenheimer, B.D.; Davé, R.; Zackrisson, E.; Thompson, R.; Huang, S. The soft, fluctuating UVB at $\mathrm{z} \sim 6$ as traced by C IV, Si IV, and C II. Mon. Not. R. Astron. Soc. 2016, 459, 2299-2310. [CrossRef]

124. Rahmati, A.; Schaye, J.; Crain, R.A.; Oppenheimer, B.D.; Schaller, M.; Theuns, T. Cosmic distribution of highly ionized metals and their physical conditions in the EAGLE simulations. Mon. Not. R. Astron. Soc. 2016, 459, 310-332. [CrossRef]

125. Bird, S.; Rubin, K.H.R.; Suresh, J.; Hernquist, L. Simulating the carbon footprint of galactic haloes. Mon. Not. R. Astron. Soc. 2016, 462, 307-322. [CrossRef]

126. Cen, R.; Chisari, N.E. Star Formation Feedback and Metal-enrichment History of the Intergalactic Medium. Astrophys. J. 2011, 731, 11. [CrossRef]

127. Cooksey, K.L.; Thom, C.; Prochaska, J.X.; Chen, H.W. The Last Eight-Billion Years of Intergalactic C IV Evolution. [CrossRef]

128. Becker, G.D.; Rauch, M.; Sargent, W.L.W. High-Redshift Metals. I. The Decline of C IV at z > 5.3. Astrophys. J. 2009, 698, 1010-1019. [CrossRef]

129. Songaila, A. The Minimum Universal Metal Density between Redshifts of 1.5 and 5.5. Astrophys. J. Lett. 2001, 561, L153-L156. [CrossRef]

130. D’Odorico, V.; Cupani, G.; Cristiani, S.; Maiolino, R.; Molaro, P.; Nonino, M.; Centurión, M.; Cimatti, A.; di Serego Alighieri, S.; Fiore, F.; et al. Metals in the IGM approaching the re-ionization epoch: Results from X-shooter at the VLT. Mon. Not. R. Astron. Soc. 2013, 435, 1198-1232. [CrossRef]

131. Matejek, M.S.; Simcoe, R.A. A Survey of Mg II Absorption at $2<\mathrm{z}<6$ with Magellan/FIRE. I. Sample and Evolution of the Mg II Frequency. Astrophys. J. 2012, 761, 112. [CrossRef]

132. McCarthy, I.G.; Schaye, J.; Ponman, T.J.; Bower, R.G.; Booth, C.M.; Dalla Vecchia, C.; Crain, R.A.; Springel, V.; Theuns, T.; Wiersma, R.P.C. The case for AGN feedback in galaxy groups. Mon. Not. R. Astron. Soc. 2010, 406, 822-839. [CrossRef]

133. Planelles, S.; Borgani, S.; Fabjan, D.; Killedar, M.; Murante, G.; Granato, G.L.; Ragone-Figueroa, C.; Dolag, K. On the role of AGN feedback on the thermal and chemodynamical properties of the hot intracluster medium. Mon. Not. R. Astron. Soc. 2014, $438,195-216$. [CrossRef]

134. Oppenheimer, B.D.; Davé, R. Cosmological simulations of intergalactic medium enrichment from galactic outflows. Mon. Not. R. Astron. Soc. 2006, 373, 1265-1292. [CrossRef]

135. Murray, N.; Quataert, E.; Thompson, T.A. On the Maximum Luminosity of Galaxies and Their Central Black Holes: Feedback from Momentum-driven Winds. Astrophys. J. 2005, 618, 569-585. [CrossRef]

136. Dalla Vecchia, C.; Schaye, J. Simulating galactic outflows with thermal supernova feedback. Mon. Not. R. Astron. Soc. 2012, 426, 140-158. [CrossRef]

137. Oppenheimer, B.D.; Davé, R. Mass, metal, and energy feedback in cosmological simulations. Mon. Not. R. Astron. Soc. 2008, 387, 577-600. [CrossRef]

138. Muratov, A.L.; Kereš, D.; Faucher-Giguère, C.A.; Hopkins, P.F.; Quataert, E.; Murray, N. Gusty, gaseous flows of FIRE: Galactic winds in cosmological simulations with explicit stellar feedback. Mon. Not. R. Astron. Soc. 2015, 454, 2691-2713. [CrossRef]

139. Hopkins, P.F.; Kereš, D.; Oñorbe, J.; Faucher-Giguère, C.A.; Quataert, E.; Murray, N.; Bullock, J.S. Galaxies on FIRE (Feedback In Realistic Environments): Stellar feedback explains cosmologically inefficient star formation. Mon. Not. R. Astron. Soc. 2014, 445, 581-603. [CrossRef]

140. Davies, J.J.; Crain, R.A.; Oppenheimer, B.D.; Schaye, J. The quenching and morphological evolution of central galaxies is facilitated by the feedback-driven expulsion of circumgalactic gas. Mon. Not. R. Astron. Soc. 2020, 491, 4462-4480. [CrossRef]

141. Stinson, G.; Seth, A.; Katz, N.; Wadsley, J.; Governato, F.; Quinn, T. Star formation and feedback in smoothed particle hydrodynamic simulations-I. Isolated galaxies. Mon. Not. R. Astron. Soc. 2006, 373, 1074-1090. [CrossRef]

142. Crain, R.A.; Theuns, T.; Dalla Vecchia, C.; Eke, V.R.; Frenk, C.S.; Jenkins, A.; Kay, S.T.; Peacock, J.A.; Pearce, F.R.; Schaye, J.; et al. Galaxies-intergalactic medium interaction calculation-I. Galaxy formation as a function of large-scale environment. Mon. Not. $R$. Astron. Soc. 2009, 399, 1773-1794. [CrossRef]

143. Bower, R.G.; Schaye, J.; Frenk, C.S.; Theuns, T.; Schaller, M.; Crain, R.A.; McAlpine, S. The dark nemesis of galaxy formation: Why hot haloes trigger black hole growth and bring star formation to an end. Mon. Not. R. Astron. Soc. 2017, 465, 32-44. [CrossRef]

144. Booth, C.M.; Schaye, J. Cosmological simulations of the growth of supermassive black holes and feedback from active galactic nuclei: Method and tests. Mon. Not. R. Astron. Soc. 2009, 398, 53-74. [CrossRef] 
145. Di Matteo, T.; Colberg, J.; Springel, V.; Hernquist, L.; Sijacki, D. Direct Cosmological Simulations of the Growth of Black Holes and Galaxies. Astrophys. J. 2008, 676, 33-53. [CrossRef]

146. Hirschmann, M.; Dolag, K.; Saro, A.; Bachmann, L.; Borgani, S.; Burkert, A. Cosmological simulations of black hole growth: AGN luminosities and downsizing. Mon. Not. R. Astron. Soc. 2014, 442, 2304-2324. [CrossRef]

147. Tremmel, M.; Governato, F.; Volonteri, M.; Quinn, T.R. Off the beaten path: A new approach to realistically model the orbital decay of supermassive black holes in galaxy formation simulations. Mon. Not. R. Astron. Soc. 2015, 451, 1868-1874. [CrossRef]

148. Springel, V.; Di Matteo, T.; Hernquist, L. Modelling feedback from stars and black holes in galaxy mergers. Mon. Not. R. Astron. Soc. 2005, 361, 776-794. [CrossRef]

149. Steinborn, L.K.; Dolag, K.; Hirschmann, M.; Prieto, M.A.; Remus, R.S. A refined sub-grid model for black hole accretion and AGN feedback in large cosmological simulations. Mon. Not. R. Astron. Soc. 2015, 448, 1504-1525. [CrossRef]

150. Gaspari, M.; Ruszkowski, M.; Oh, S.P. Chaotic cold accretion on to black holes. Mon. Not. R. Astron. Soc. 2013, 432, 3401-3422. [CrossRef]

151. Rosas-Guevara, Y.M.; Bower, R.G.; Schaye, J.; Furlong, M.; Frenk, C.S.; Booth, C.M.; Crain, R.A.; Dalla Vecchia, C.; Schaller, M.; Theuns, T. The impact of angular momentum on black hole accretion rates in simulations of galaxy formation. Mon. Not. $R$. Astron. Soc. 2015, 454, 1038-1057. [CrossRef]

152. Hopkins, P.F.; Quataert, E. An analytic model of angular momentum transport by gravitational torques: From galaxies to massive black holes. Mon. Not. R. Astron. Soc. 2011, 415, 1027-1050. [CrossRef]

153. Anglés-Alcázar, D.; Davé, R.; Faucher-Giguère, C.A.; Özel, F.; Hopkins, P.F. Gravitational torque-driven black hole growth and feedback in cosmological simulations. Mon. Not. R. Astron. Soc. 2017, 464, 2840-2853. [CrossRef]

154. Davé, R.; Crain, R.A.; Stevens, A.R.H.; Narayanan, D.; Saintonge, A.; Catinella, B.; Cortese, L. Galaxy cold gas contents in modern cosmological hydrodynamic simulations. Mon. Not. R. Astron. Soc. 2020, 497, 146-166. [CrossRef]

155. Lin, Y.T.; Mohr, J.J. K-band Properties of Galaxy Clusters and Groups: Brightest Cluster Galaxies and Intracluster Light. Astrophys. J. 2004, 617, 879-895. [CrossRef]

156. Shakura, N.I.; Sunyaev, R.A. Reprint of 1973A\&A....24..337S. Black holes in binary systems. Observational appearance. Astron. Astrophys. 1973, 500, 33-51.

157. Weinberger, R.; Springel, V.; Hernquist, L.; Pillepich, A.; Marinacci, F.; Pakmor, R.; Nelson, D.; Genel, S.; Vogelsberger, M.; Naiman, J.; et al. Simulating galaxy formation with black hole driven thermal and kinetic feedback. Mon. Not. R. Astron. Soc. 2017, 465, 3291-3308. [CrossRef]

158. McCarthy, I.G.; Schaye, J.; Bower, R.G.; Ponman, T.J.; Booth, C.M.; Dalla Vecchia, C.; Springel, V. Gas expulsion by quasar-driven winds as a solution to the overcooling problem in galaxy groups and clusters. Mon. Not. R. Astron. Soc. 2011, 412, 1965-1984. [CrossRef]

159. Sijacki, D.; Springel, V.; Di Matteo, T.; Hernquist, L. A unified model for AGN feedback in cosmological simulations of structure formation. Mon. Not. R. Astron. Soc. 2007, 380, 877-900. [CrossRef]

160. Genel, S.; Vogelsberger, M.; Springel, V.; Sijacki, D.; Nelson, D.; Snyder, G.; Rodriguez-Gomez, V.; Torrey, P.; Hernquist, L. Introducing the Illustris project: The evolution of galaxy populations across cosmic time. Mon. Not. R. Astron. Soc. 2014, 445, 175-200. [CrossRef]

161. Dubois, Y.; Devriendt, J.; Slyz, A.; Teyssier, R. Self-regulated growth of supermassive black holes by a dual jet-heating active galactic nucleus feedback mechanism: Methods, tests and implications for cosmological simulations. Mon. Not. R. Astron. Soc. 2012, 420, 2662-2683. [CrossRef]

162. Davies, J.J.; Crain, R.A.; McCarthy, I.G.; Oppenheimer, B.D.; Schaye, J.; Schaller, M.; McAlpine, S. The gas fractions of dark matter haloes hosting simulated $\sim \mathrm{L}^{\star}$ galaxies are governed by the feedback history of their black holes. Mon. Not. R. Astron. Soc. 2019, 485, 3783-3793. [CrossRef]

163. Terrazas, B.A.; Bell, E.F.; Pillepich, A.; Nelson, D.; Somerville, R.S.; Genel, S.; Weinberger, R.; Habouzit, M.; Li, Y.; Hernquist, L.; et al. The relationship between black hole mass and galaxy properties: Examining the black hole feedback model in IllustrisTNG. Mon. Not. R. Astron. Soc. 2020, 493, 1888-1906. [CrossRef]

164. Nulsen, P.E.J. Transport processes and the stripping of cluster galaxies. Mon. Not. R. Astron. Soc. 1982, 198, 1007-1016. [CrossRef]

165. Sijacki, D.; Springel, V. Physical viscosity in smoothed particle hydrodynamics simulations of galaxy clusters. Mon. Not. R. Astron. Soc. 2006, 371, 1025-1046. [CrossRef]

166. Hopkins, P.F. Anisotropic diffusion in mesh-free numerical magnetohydrodynamics. Mon. Not. R. Astron. Soc. 2017, 466, 33873405. [CrossRef]

167. Barnes, D.J.; Kannan, R.; Vogelsberger, M.; Pfrommer, C.; Puchwein, E.; Weinberger, R.; Springel, V.; Pakmor, R.; Nelson, D.; Marinacci, F.; et al. Enhancing AGN efficiency and cool-core formation with anisotropic thermal conduction. Mon. Not. R. Astron. Soc. 2019, 488, 3003-3013. [CrossRef]

168. Rennehan, D.; Babul, A.; Hopkins, P.F.; Davé, R.; Moa, B. Dynamic localized turbulent diffusion and its impact on the galactic ecosystem. Mon. Not. R. Astron. Soc. 2019, 483, 3810-3831. [CrossRef]

169. Fabian, A.C.; Sanders, J.S.; Ettori, S.; Taylor, G.B.; Allen, S.W.; Crawford, C.S.; Iwasawa, K.; Johnstone, R.M.; Ogle, P.M. Chandra imaging of the complex X-ray core of the Perseus cluster. Mon. Not. R. Astron. Soc. 2000, 318, L65-L68. [CrossRef]

170. Brüggen, M.; Ruszkowski, M.; Hallman, E. Active Galactic Nuclei Heating and Dissipative Processes in Galaxy Clusters. Astrophys. J. 2005, 630, 740-749. [CrossRef] 
171. Dursi, L.J.; Pfrommer, C. Draping of Cluster Magnetic Fields over Bullets and Bubbles-Morphology and Dynamic Effects. Astrophys. J. 2008, 677, 993-1018. [CrossRef]

172. Fabian, A.C.; Sanders, J.S.; Allen, S.W.; Crawford, C.S.; Iwasawa, K.; Johnstone, R.M.; Schmidt, R.W.; Taylor, G.B. A deep Chandra observation of the Perseus cluster: Shocks and ripples. Mon. Not. R. Astron. Soc. 2003, 344, L43-L47. [CrossRef]

173. Ruszkowski, M.; Brüggen, M.; Begelman, M.C. Three-Dimensional Simulations of Viscous Dissipation in the Intracluster Medium. Astrophys. J. 2004, 615, 675-680. [CrossRef]

174. Jiang, Y.F.; Oh, S.P. A New Numerical Scheme for Cosmic-Ray Transport. Astrophys. J. 2018, 854, 5. [CrossRef]

175. Chan, T.K.; Kereš, D.; Hopkins, P.F.; Quataert, E.; Su, K.Y.; Hayward, C.C.; Faucher-Giguère, C.A. Cosmic ray feedback in the FIRE simulations: Constraining cosmic ray propagation with $\mathrm{GeV} \gamma$-ray emission. Mon. Not. R. Astron. Soc. 2019, 488, 3716-3744. [CrossRef]

176. Thomas, T.; Pfrommer, C. Cosmic-ray hydrodynamics: Alfvén-wave regulated transport of cosmic rays. Mon. Not. R. Astron. Soc. 2019, 485, 2977-3008. [CrossRef]

177. Zweibel, E.G. The microphysics and macrophysics of cosmic rays. Phys. Plasmas 2013, 20, 055501. [CrossRef]

178. Zweibel, E.G. The basis for cosmic ray feedback: Written on the wind. Phys. Plasmas 2017, 24, 055402. [CrossRef]

179. Wiener, J.; Pfrommer, C.; Oh, S.P. Cosmic ray-driven galactic winds: Streaming or diffusion? Mon. Not. R. Astron. Soc. 2017, $467,906-921$.

[CrossRef]

180. Butsky, I.S.; Quinn, T.R. The Role of Cosmic-ray Transport in Shaping the Simulated Circumgalactic Medium. Astrophys. J. 2018, 868, 108. [CrossRef]

181. Hopkins, P.F.; Chan, T.K.; Squire, J.; Quataert, E.; Ji, S.; Kereš, D.; Faucher-Giguère, C.A. Effects of different cosmic ray transport models on galaxy formation. Mon. Not. R. Astron. Soc. 2021, 501, 3663-3669. [CrossRef]

182. Ipavich, F.M. Galactic winds driven by cosmic rays. Astrophys. J. 1975, 196, 107-120. [CrossRef]

183. Uhlig, M.; Pfrommer, C.; Sharma, M.; Nath, B.B.; Enßlin, T.A.; Springel, V. Galactic winds driven by cosmic ray streaming. Mon. Not. R. Astron. Soc. 2012, 423, 2374-2396. [CrossRef]

184. Pakmor, R.; Pfrommer, C.; Simpson, C.M.; Springel, V. Galactic Winds Driven by Isotropic and Anisotropic Cosmic-Ray Diffusion in Disk Galaxies. Astrophys. J. Lett. 2016, 824, L30. [CrossRef]

185. Ruszkowski, M.; Yang, H.Y.K.; Zweibel, E. Global Simulations of Galactic Winds Including Cosmic-ray Streaming. Astrophys. J. 2017, 834, 208. [CrossRef]

186. Hopkins, P.F.; Chan, T.K.; Ji, S.; Hummels, C.B.; Kereš, D.; Quataert, E.; Faucher-Giguère, C.A. Cosmic ray driven outflows to Mpc scales from $\mathrm{L}_{*}$ galaxies. Mon. Not. R. Astron. Soc. 2021, 501, 3640-3662. [CrossRef]

187. Girichidis, P.; Naab, T.; Hanasz, M.; Walch, S. Cooler and smoother-The impact of cosmic rays on the phase structure of galactic outflows. Mon. Not. R. Astron. Soc. 2018, 479, 3042-3067. [CrossRef]

188. Jacob, S.; Pakmor, R.; Simpson, C.M.; Springel, V.; Pfrommer, C. The dependence of cosmic ray-driven galactic winds on halo mass. Mon. Not. R. Astron. Soc. 2018, 475, 570-584. [CrossRef]

189. Guo, F.; Oh, S.P. Feedback heating by cosmic rays in clusters of galaxies. Mon. Not. R. Astron. Soc. 2008, 384, 251-266. [CrossRef]

190. Enßlin, T.; Pfrommer, C.; Miniati, F.; Subramanian, K. Cosmic ray transport in galaxy clusters: Implications for radio halos, gamma-ray signatures, and cool core heating. Astron. Astrophys. 2011, 527, A99. [CrossRef]

191. Wiener, J.; Oh, S.P.; Guo, F. Cosmic ray streaming in clusters of galaxies. Mon. Not. R. Astron. Soc. 2013, 434, 2209-2228. [CrossRef]

192. Su, K.Y.; Hopkins, P.F.; Hayward, C.C.; Faucher-Giguère, C.A.; Kereš, D.; Ma, X.; Orr, M.E.; Chan, T.K.; Robles, V.H. Cosmic rays or turbulence can suppress cooling flows (where thermal heating or momentum injection fail). Mon. Not. R. Astron. Soc. 2020, 491, 1190-1212. [CrossRef]

193. Jacob, S.; Pfrommer, C. Cosmic ray heating in cool core clusters II: Self-regulation cycle and non-thermal emission. Mon. Not. R. Astron. Soc. 2017. [CrossRef]

194. Salem, M.; Bryan, G.L.; Corlies, L. Role of cosmic rays in the circumgalactic medium. Mon. Not. R. Astron. Soc. 2016, $456,582-601$. [CrossRef]

195. Ji, S.; Chan, T.K.; Hummels, C.B.; Hopkins, P.F.; Stern, J.; Kereš, D.; Quataert, E.; Faucher-Giguère, C.A.; Murray, N. Properties of the circumgalactic medium in cosmic ray-dominated galaxy haloes. Mon. Not. R. Astron. Soc. 2020, 496, 4221-4238. [CrossRef]

196. Buck, T.; Pfrommer, C.; Pakmor, R.; Grand, R.J.J.; Springel, V. The effects of cosmic rays on the formation of Milky Way-mass galaxies in a cosmological context. Mon. Not. R. Astron. Soc. 2020, 497, 1712-1737. [CrossRef]

197. Sharma, P.; Parrish, I.J.; Quataert, E. Thermal Instability with Anisotropic Thermal Conduction and Adiabatic Cosmic Rays: Implications for Cold Filaments in Galaxy Clusters. Astrophys. J. 2010, 720, 652-665. [CrossRef]

198. Ruszkowski, M.; Yang, H.Y.K.; Reynolds, C.S. Powering of H $\alpha$ Filaments by Cosmic Rays. Astrophys. J. 2018, 858, 64. [CrossRef]

199. Butsky, I.S.; Fielding, D.B.; Hayward, C.C.; Hummels, C.B.; Quinn, T.R.; Werk, J.K. The Impact of Cosmic Rays on Thermal Instability in the Circumgalactic Medium. Astrophys. J. 2020, 903, 77. [CrossRef]

200. Helsdon, S.F.; Ponman, T.J. The intragroup medium in loose groups of galaxies. Mon. Not. R. Astron. Soc. 2000, 315, 356-370. [CrossRef]

201. White, D.A.; Jones, C.; Forman, W. An investigation of cooling flows and general cluster properties from an X-ray image deprojection analysis of 207 clusters of galaxies. Mon. Not. R. Astron. Soc. 1997, 292, 419-467. [CrossRef] 
202. Balogh, M.L.; Babul, A.; Patton, D.R. Pre-heated isentropic gas in groups of galaxies. Mon. Not. R. Astron. Soc. 1999, 307, 463-479. [CrossRef]

203. Bryan, G.L. Explaining the Entropy Excess in Clusters and Groups of Galaxies without Additional Heating. Astrophys. J. Lett. 2000, 544, L1-L5. [CrossRef]

204. Davé, R.; Katz, N.; Weinberg, D.H. X-Ray Scaling Relations of Galaxy Groups in a Hydrodynamic Cosmological Simulation. Astrophys. J. 2002, 579, 23-41. [CrossRef]

205. Gonzalez, A.H.; Zaritsky, D.; Zabludoff, A.I. A Census of Baryons in Galaxy Clusters and Groups. Astrophys. J. 2007, 666, 147-155. [CrossRef]

206. Balogh, M.L.; McCarthy, I.G.; Bower, R.G.; Eke, V.R. Testing cold dark matter with the hierarchical build-up of stellar light. Mon. Not. R. Astron. Soc. 2008, 385, 1003-1014. [CrossRef]

207. Kravtsov, A.V.; Vikhlinin, A.A.; Meshcheryakov, A.V. Stellar Mass-Halo Mass Relation and Star Formation Efficiency in High-Mass Halos. Astron. Lett. 2018, 44, 8-34. [CrossRef]

208. Ponman, T.J.; Sanderson, A.J.R.; Finoguenov, A. The Birmingham-CfA cluster scaling project-III. Entropy and similarity in galaxy systems. Mon. Not. R. Astron. Soc. 2003, 343, 331-342. [CrossRef]

209. Fabian, A.C.; Nulsen, P.E.J.; Canizares, C.R. Cooling flows in clusters of galaxies. Nature 1984, 310, 733-740. [CrossRef]

210. Giodini, S.; Pierini, D.; Finoguenov, A.; Pratt, G.W.; Boehringer, H.; Leauthaud, A.; Guzzo, L.; Aussel, H.; Bolzonella, M.; Capak, P.; et al. Stellar and Total Baryon Mass Fractions in Groups and Clusters Since Redshift 1. Astrophys. J. 2009, 703, 982-993. [CrossRef]

211. Schaller, M.; Frenk, C.S.; Bower, R.G.; Theuns, T.; Jenkins, A.; Schaye, J.; Crain, R.A.; Furlong, M.; Dalla Vecchia, C.; McCarthy, I.G. Baryon effects on the internal structure of $\Lambda$ CDM haloes in the EAGLE simulations. Mon. Not. R. Astron. Soc. 2015, 451, 1247-1267. [CrossRef]

212. Sun, M.; Sehgal, N.; Voit, G.M.; Donahue, M.; Jones, C.; Forman, W.; Vikhlinin, A.; Sarazin, C. The Pressure Profiles of Hot Gas in Local Galaxy Groups. Astrophys. J. Lett. 2011, 727, L49. [CrossRef]

213. Babul, A.; Balogh, M.L.; Lewis, G.F.; Poole, G.B. Physical implications of the X-ray properties of galaxy groups and clusters. Mon. Not. R. Astron. Soc. 2002, 330, 329-343. [CrossRef]

214. Barnes, D.J.; Vogelsberger, M.; Kannan, R.; Marinacci, F.; Weinberger, R.; Springel, V.; Torrey, P.; Pillepich, A.; Nelson, D.; Pakmor, R.; et al. A census of cool-core galaxy clusters in IllustrisTNG. Mon. Not. R. Astron. Soc. 2018, 481, 1809-1831. [CrossRef]

215. Robson, D.; Davé, R. X-ray emission from hot gas in galaxy groups and clusters in SIMBA. Mon. Not. R. Astron. Soc. 2020, 498, 3061-3076. [CrossRef]

216. McCarthy, I.G.; Babul, A.; Bower, R.G.; Balogh, M.L. Towards a holistic view of the heating and cooling of the intracluster medium. Mon. Not. R. Astron. Soc. 2008, 386, 1309-1331. [CrossRef]

217. Gitti, M.; Nulsen, P.E.J.; David, L.P.; McNamara, B.R.; Wise, M.W. A Chandra Study of the Large-scale Shock and Cool Filaments in Hydra A: Evidence for Substantial Gas Dredge-up by the Central Outburst. Astrophys. J. 2011, 732, 13. [CrossRef]

218. Vantyghem, A.N.; McNamara, B.R.; Russell, H.R.; Main, R.A.; Nulsen, P.E.J.; Wise, M.W.; Hoekstra, H.; Gitti, M. Cycling of the powerful AGN in MS $0735.6+7421$ and the duty cycle of radio AGN in clusters. Mon. Not. R. Astron. Soc. 2014, 442, 3192-3205. [CrossRef]

219. David, L.P.; Nulsen, P.E.J.; McNamara, B.R.; Forman, W.; Jones, C.; Ponman, T.; Robertson, B.; Wise, M. A High-Resolution Study of the Hydra A Cluster with Chandra: Comparison of the Core Mass Distribution with Theoretical Predictions and Evidence for Feedback in the Cooling Flow. Astrophys. J. 2001, 557, 546-559. [CrossRef]

220. Hlavacek-Larrondo, J.; McDonald, M.; Benson, B.A.; Forman, W.R.; Allen, S.W.; Bleem, L.E.; Ashby, M.L.N.; Bocquet, S.; Brodwin, M.; Dietrich, J.P.; et al. X-Ray Cavities in a Sample of 83 SPT-selected Clusters of Galaxies: Tracing the Evolution of AGN Feedback in Clusters of Galaxies out to $\mathrm{z}=1.2$. Astrophys. J. 2015, 805, 35. [CrossRef]

221. Cavaliere, A.; Menci, N.; Tozzi, P. Diffuse Baryons in Groups and Clusters of Galaxies. Astrophys. J. 1998, 501, 493-508. [CrossRef]

222. Voit, G.M.; Bryan, G.L. Regulation of the X-ray luminosity of clusters of galaxies by cooling and supernova feedback. Nature 2001, 414, 425-427. [CrossRef]

223. Voit, G.M.; Bryan, G.L.; Balogh, M.L.; Bower, R.G. Modified Entropy Models for the Intracluster Medium. Astrophys. J. 2002, 576, 601-624. [CrossRef]

224. Borgani, S.; Murante, G.; Springel, V.; Diaferio, A.; Dolag, K.; Moscardini, L.; Tormen, G.; Tornatore, L.; Tozzi, P. X-ray properties of galaxy clusters and groups from a cosmological hydrodynamical simulation. Mon. Not. R. Astron. Soc. 2004, 348, 1078-1096. [CrossRef]

225. Brough, S.; Forbes, D.A.; Kilborn, V.A.; Couch, W. Southern GEMS groups-I. Dynamical properties. Mon. Not. R. Astron. Soc. 2006, 370, 1223-1246. [CrossRef]

226. Brough, S.; Couch, W.J.; Collins, C.A.; Jarrett, T.; Burke, D.J.; Mann, R.G. The luminosity-halo mass relation for brightest cluster galaxies. Mon. Not. R. Astron. Soc. 2008, 385, L103-L107. [CrossRef]

227. Von Der Linden, A.; Best, P.N.; Kauffmann, G.; White, S.D.M. How special are brightest group and cluster galaxies? Mon. Not. R. Astron. Soc. 2007, 379, 867-893. [CrossRef]

228. Weinmann, S.M.; van den Bosch, F.C.; Yang, X.; Mo, H.J. Properties of galaxy groups in the Sloan Digital Sky Survey-I. The dependence of colour, star formation and morphology on halo mass. Mon. Not. R. Astron. Soc. 2006, 366, 2-28. [CrossRef] 
229. Gozaliasl, G.; Finoguenov, A.; Khosroshahi, H.G.; Mirkazemi, M.; Erfanianfar, G.; Tanaka, M. Brightest group galaxies: Stellar mass and star formation rate (paper I). Mon. Not. R. Astron. Soc. 2016, 458, 2762-2775. [CrossRef]

230. Cougo, J.; Rembold, S.B.; Ferrari, F.; Kaipper, A.L.P. Morphometric analysis of brightest cluster galaxies. Mon. Not. R. Astron. Soc. 2020, 498, 4433-4449. [CrossRef]

231. Loubser, S.I.; Hoekstra, H.; Babul, A.; O'Sullivan, E. Diversity in the stellar velocity dispersion profiles of a large sample of brightest cluster galaxies z $\leq$ 0.3. Mon. Not. R. Astron. Soc. 2018, 477, 335-358. [CrossRef]

232. Murante, G.; Giovalli, M.; Gerhard, O.; Arnaboldi, M.; Borgani, S.; Dolag, K. The importance of mergers for the origin of intracluster stars in cosmological simulations of galaxy clusters. Mon. Not. R. Astron. Soc. 2007, 377, 2-16. [CrossRef]

233. Dubinski, J. The Origin of the Brightest Cluster Galaxies. Astrophys. J. 1998, 502, 141-149. [CrossRef]

234. De Lucia, G.; Blaizot, J. The hierarchical formation of the brightest cluster galaxies. Mon. Not. R. Astron. Soc. 2007, 375, 2-14. [CrossRef]

235. Cooper, A.P.; Gao, L.; Guo, Q.; Frenk, C.S.; Jenkins, A.; Springel, V.; White, S.D.M. Surface photometry of brightest cluster galaxies and intracluster stars in $\Lambda$ CDM. Mon. Not. R. Astron. Soc. 2015, 451, 2703-2722. [CrossRef]

236. Nipoti, C. The special growth history of central galaxies in groups and clusters. Mon. Not. R. Astron. Soc. 2017, 467, 661-673. [CrossRef]

237. Ragone-Figueroa, C.; Granato, G.L.; Ferraro, M.E.; Murante, G.; Biffi, V.; Borgani, S.; Planelles, S.; Rasia, E. BCG mass evolution in cosmological hydro-simulations. Mon. Not. R. Astron. Soc. 2018, 479, 1125-1136. [CrossRef]

238. Clauwens, B.; Schaye, J.; Franx, M.; Bower, R.G. The three phases of galaxy formation. Mon. Not. R. Astron. Soc. 2018, 478, 3994-4009. [CrossRef]

239. Davison, T.A.; Norris, M.A.; Pfeffer, J.L.; Davies, J.J.; Crain, R.A. An EAGLE's view of ex situ galaxy growth. Mon. Not. R. Astron. Soc. 2020, 497, 81-93. [CrossRef]

240. Henden, N.A.; Puchwein, E.; Sijacki, D. The baryon content of groups and clusters of galaxies in the FABLE simulations. Mon. Not. R. Astron. Soc. 2020, 498, 2114-2137. [CrossRef]

241. Jackson, T.M.; Pasquali, A.; Pacifici, C.; Engler, C.; Pillepich, A.; Grebel, E.K. The stellar mass assembly of low-redshift, massive, central galaxies in SDSS and the TNG300 simulation. Mon. Not. R. Astron. Soc. 2020, 497, 4262-4275. [CrossRef]

242. Katsianis, A.; Xu, H.; Yang, X.; Luo, Y.; Cui, W.; Davé, R.; Lagos, C.D.P.; Zheng, X.; Zhao, P. The specific star formation rate function at different mass scales and quenching: A comparison between cosmological models and SDSS. Mon. Not. R. Astron. Soc. 2021, 500, 2036-2048. [CrossRef]

243. Remus, R.S.; Dolag, K.; Hoffmann, T. The Outer Halos of Very Massive Galaxies: BCGs and their DSC in the Magneticum Simulations. Galaxies 2017, 5, 49. [CrossRef]

244. Tacchella, S.; Diemer, B.; Hernquist, L.; Genel, S.; Marinacci, F.; Nelson, D.; Pillepich, A.; Rodriguez-Gomez, V.; Sales, L.V.; Springel, V.; Vogelsberger, M. Morphology and star formation in IllustrisTNG: The build-up of spheroids and discs. Mon. Not. R. Astron. Soc. 2019, 487, 5416-5440. [CrossRef]

245. Gonzalez, A.H.; Sivanandam, S.; Zabludoff, A.I.; Zaritsky, D. Galaxy Cluster Baryon Fractions Revisited. Astrophys. J. 2013, 778, 14. [CrossRef]

246. Coupon, J.; Arnouts, S.; van Waerbeke, L.; Moutard, T.; Ilbert, O.; van Uitert, E.; Erben, T.; Garilli, B.; Guzzo, L.; Heymans, C.; et al. The galaxy-halo connection from a joint lensing, clustering and abundance analysis in the CFHTLenS/VIPERS field. Mon. Not. R. Astron. Soc. 2015, 449, 1352-1379. [CrossRef]

247. Girelli, G.; Pozzetti, L.; Bolzonella, M.; Giocoli, C.; Marulli, F.; Baldi, M. The stellar-to-halo mass relation over the past 12 Gyr. I. Standard $\Lambda$ CDM model. Astron. Astrophys. 2020, 634, A135. [CrossRef]

248. Yang, X.; Mo, H.J.; van den Bosch, F.C. Galaxy Groups in the SDSS DR4. II. Halo Occupation Statistics. Astrophys. J. 2008, 676, 248-261. [CrossRef]

249. Yang, X.; Mo, H.J.; van den Bosch, F.C.; Zhang, Y.; Han, J. Evolution of the Galaxy-Dark Matter Connection and the Assembly of Galaxies in Dark Matter Halos. Astrophys. J. 2012, 752, 41. [CrossRef]

250. Moster, B.P.; Naab, T.; White, S.D.M. Galactic star formation and accretion histories from matching galaxies to dark matter haloes. Mon. Not. R. Astron. Soc. 2013, 428, 3121-3138. [CrossRef]

251. Van Uitert, E.; Cacciato, M.; Hoekstra, H.; Brouwer, M.; Sifón, C.; Viola, M.; Baldry, I.; Bland-Hawthorn, J.; Brough, S.; Brown, M.J.I.; et al. The stellar-to-halo mass relation of GAMA galaxies from $100 \mathrm{deg}^{2}$ of KiDS weak lensing data. Mon. Not. R. Astron. Soc. 2016, 459, 3251-3270. [CrossRef]

252. Erfanianfar, G.; Finoguenov, A.; Furnell, K.; Popesso, P.; Biviano, A.; Wuyts, S.; Collins, C.A.; Mirkazemi, M.; Comparat, J.; Khosroshahi, H.; et al. Stellar mass-halo mass relation for the brightest central galaxies of X-ray clusters since $\mathrm{z} \sim 0.65$. Astron. Astrophys. 2019, 631, A175. [CrossRef]

253. Kolokythas, K.; Vaddi, S. Star-formation \& galaxy growth in the dominant galaxies of CLoGS sample: Feedback implications in Local Universe galaxy groups. In preparation.

254. Sand, D.J.; Graham, M.L.; Bildfell, C.; Zaritsky, D.; Pritchet, C.; Hoekstra, H.; Just, D.W.; Herbert-Fort, S.; Sivanandam, S.; Foley, R.J.; et al. The Multi-Epoch nearby Cluster Survey: Type Ia Supernova Rate Measurement in z 0.1 Clusters and the Late-time Delay Time Distribution. Astrophys. J. 2012, 746, 163. [CrossRef]

255. Bildfell, C.; Hoekstra, H.; Babul, A.; Mahdavi, A. Resurrecting the red from the dead: Optical properties of BCGs in X-ray luminous clusters. Mon. Not. R. Astron. Soc. 2008, 389, 1637-1654. [CrossRef] 
256. Mahdavi, A.; Hoekstra, H.; Babul, A.; Bildfell, C.; Jeltema, T.; Henry, J.P. Joint Analysis of Cluster Observations. II. Chandra/XMMNewton X-Ray and Weak Lensing Scaling Relations for a Sample of 50 Rich Clusters of Galaxies. Astrophys. J. 2013, $767,116$. [CrossRef]

257. Hoekstra, H.; Herbonnet, R.; Muzzin, A.; Babul, A.; Mahdavi, A.; Viola, M.; Cacciato, M. The Canadian Cluster Comparison Project: Detailed study of systematics and updated weak lensing masses. Mon. Not. R. Astron. Soc. 2015, 449, 685-714. [CrossRef]

258. Loubser, S.I.; Babul, A.; Hoekstra, H.; Mahdavi, A.; Donahue, M.; Bildfell, C.; Voit, G.M. The regulation of star formation in cool-core clusters: Imprints on the stellar populations of brightest cluster galaxies. Mon. Not. R. Astron. Soc. 2016, 456, 1565-1578. [CrossRef]

259. Herbonnet, R.; Sifón, C.; Hoekstra, H.; Bahé, Y.; van der Burg, R.F.J.; Melin, J.B.; von der Linden, A.; Sand, D.; Kay, S.; Barnes, D. CCCP and MENeaCS: (updated) weak-lensing masses for 100 galaxy clusters. Mon. Not. R. Astron. Soc. 2020, 497, 4684-4703. [CrossRef]

260. Mittal, R.; Whelan, J.T.; Combes, F. Constraining star formation rates in cool-core brightest cluster galaxies. Mon. Not. R. Astron. Soc. 2015, 450, 2564-2592. [CrossRef]

261. Cooke, K.C.; Fogarty, K.; Kartaltepe, J.S.; Moustakas, J.; O’Dea, C.P.; Postman, M. Stellar Mass and $3.4 \mu \mathrm{m}$ M/L Ratio Evolution of Brightest Cluster Galaxies in COSMOS since $\mathrm{z} \sim 1.0$. Astrophys. J. 2018, 857, 122. [CrossRef]

262. Whitaker, K.E.; van Dokkum, P.G.; Brammer, G.; Franx, M. The Star Formation Mass Sequence Out to z = 2.5. Astrophys. J. Lett. 2012, 754, L29. [CrossRef]

263. McDonald, M.; Veilleux, S.; Rupke, D.S.N.; Mushotzky, R.; Reynolds, C. Star formation efficiency in the cool cores of galaxy clusters. Astrophys. J. 2011, 734, 95. [CrossRef]

264. McCarthy, I.G.; Balogh, M.L.; Babul, A.; Poole, G.B.; Horner, D.J. Models of the Intracluster Medium with Heating and Cooling: Explaining the Global and Structural X-Ray Properties of Clusters. Astrophys. J. 2004, 613, 811-830. [CrossRef]

265. Andrade-Santos, F.; Jones, C.; Forman, W.R.; Lovisari, L.; Vikhlinin, A.; Weeren, R.J.V.; Murray, S.S.; Kraft, R.; Mazzotta, P.; David, L.; et al. The Fraction of Cool-core Clusters in X-ray versus SZ Samples Using Chandra Observations. Astrophys. J. 2017, 843. [CrossRef]

266. Bonjean, V.; Aghanim, N.; Salomé, P.; Beelen, A.; Douspis, M.; Soubrié, E. Star formation rates and stellar masses from machine learning. Astron. Astrophys. 2019,622, A137. [CrossRef]

267. Kennicutt, R.C.; Evans, N.J. Star Formation in the Milky Way and Nearby Galaxies. Annu. Rev. Astron. Astrophys. 2012, 50, 531-608. [CrossRef]

268. Pipino, A.; Kaviraj, S.; Bildfell, C.; Babul, A.; Hoekstra, H.; Silk, J. Evidence for recent star formation in BCGs: A correspondence between blue cores and UV excess. Mon. Not. R. Astron. Soc. 2009, 395, 462-471. [CrossRef]

269. Fogarty, K.; Postman, M.; Larson, R.; Donahue, M.; Moustakas, J. The Relationship Between Brightest Cluster Galaxy Star Formation and the Intracluster Medium in CLASH. Astrophys. J. 2017, 846, 103. [CrossRef]

270. Mittal, R.; McDonald, M.; Whelan, J.T.; Bruzual, G. The challenging task of determining star formation rates: The case of a massive stellar burst in the brightest cluster galaxy of Phoenix galaxy cluster. Mon. Not. R. Astron. Soc. 2017, 465, 3143-3153. [CrossRef]

271. Loubser, S.I.; Sánchez-Blázquez, P. The ultraviolet upturn in brightest cluster galaxies. Mon. Not. R. Astron. Soc. 2011, 410, 2679-2689. [CrossRef]

272. Li, Y.T.; Chen, L.W. First ranked galaxies of non-elliptical morphology. Mon. Not. R. Astron. Soc. 2019, 482, 4084-4095. [CrossRef]

273. Scannapieco, C.; Gadotti, D.A.; Jonsson, P.; White, S.D.M. An observer's view of simulated galaxies: Disc-to-total ratios, bars and (pseudo-)bulges. Mon. Not. R. Astron. Soc. 2010, 407, L41-L45. [CrossRef]

274. Bottrell, C.; Torrey, P.; Simard, L.; Ellison, S.L. Galaxies in the Illustris simulation as seen by the Sloan Digital Sky Survey-II Size-luminosity relations and the deficit of bulge-dominated galaxies in Illustris at low mass. Mon. Not. R. Astron. Soc. 2017, 467, 2879-2895. [CrossRef]

275. Zhu, L.; van de Ven, G.; Méndez-Abreu, J.; Obreja, A. Morphology and kinematics of orbital components in CALIFA galaxies across the Hubble sequence. Mon. Not. R. Astron. Soc. 2018, 479, 945-960. [CrossRef]

276. Valageas, P.; Silk, J. The entropy history of the universe. Astron. Astrophys. 1999, 350, 725-742.

277. Nath, B.B.; Roychowdhury, S. Heating of the intracluster medium by quasar outflows. Mon. Not. R. Astron. Soc. 2002, 333, 145-155. [CrossRef]

278. Pointon, S.K.; Nielsen, N.M.; Kacprzak, G.G.; Muzahid, S.; Churchill, C.W.; Charlton, J.C. The Impact of the Group Environment on the O VI Circumgalactic Medium. Astrophys. J. 2017, 844, 23. [CrossRef]

279. Nielsen, N.M.; Kacprzak, G.G.; Pointon, S.K.; Churchill, C.W.; Murphy, M.T. MAGIICAT VI. The Mg II Intragroup Medium Is Kinematically Complex. Astrophys. J. 2018, 869, 153. [CrossRef]

280. Yoon, J.H.; Putman, M.E.; Thom, C.; Chen, H.W.; Bryan, G.L. Warm Gas in the Virgo Cluster. I. Distribution of Ly $\alpha$ Absorbers. Astrophys. J. 2012, 754, 84. [CrossRef]

281. Manuwal, A.; Narayanan, A.; Muzahid, S.; Charlton, J.C.; Khaire, V.; Chand, H. C IV absorbers tracing cool gas in dense galaxy group/cluster environments. Mon. Not. R. Astron. Soc. 2019, 485, 30-46. [CrossRef]

282. Yoon, J.H.; Putman, M.E. Ly $\alpha$ Absorbers and the Coma Cluster. Astrophys. J. 2017, 839, 117. [CrossRef]

283. Connor, T.; Zahedy, F.S.; Chen, H.W.; Cooper, T.J.; Mulchaey, J.S.; Vikhlinin, A. COS Observations of the Cosmic Web: A Search for the Cooler Components of a Hot, X-Ray Identified Filament. Astrophys. J. Lett. 2019, 884, L20. [CrossRef] 
284. Muzahid, S.; Charlton, J.; Nagai, D.; Schaye, J.; Srianand, R. Discovery of an H i-rich Gas Reservoir in the Outskirts of SZ-effect-selected Clusters. Astrophys. J. Lett. 2017, 846, L8. [CrossRef]

285. Pradeep, J.; Narayanan, A.; Muzahid, S.; Nagai, D.; Charlton, J.C.; Srianand, R. Detection of metal-rich, cool-warm gas in the outskirts of galaxy clusters. Mon. Not. R. Astron. Soc. 2019, 488, 5327-5339. [CrossRef]

286. Zahedy, F.S.; Chen, H.W.; Johnson, S.D.; Pierce, R.M.; Rauch, M.; Huang, Y.H.; Weiner, B.J.; Gauthier, J.R. Characterizing circumgalactic gas around massive ellipticals at $\mathrm{z} \sim 0.4-$ II. Physical properties and elemental abundances. Mon. Not. R. Astron. Soc. 2019, 484, 2257-2280. [CrossRef]

287. Biffi, V.; Planelles, S.; Borgani, S.; Rasia, E.; Murante, G.; Fabjan, D.; Gaspari, M. The origin of ICM enrichment in the outskirts of present-day galaxy clusters from cosmological hydrodynamical simulations. Mon. Not. R. Astron. Soc. 2018, 476, 2689-2703. [CrossRef]

288. McCourt, M.; Sharma, P.; Quataert, E.; Parrish, I.J. Thermal instability in gravitationally stratified plasmas: implications for multiphase structure in clusters and galaxy haloes. Mon. Not. R. Astron. Soc. 2012, 419, 3319-3337. [CrossRef]

289. Esmerian, C.J.; Kravtsov, A.V.; Hafen, Z.; Faucher-Giguère, C.A.; Quataert, E.; Stern, J.; Kereš, D.; Wetzel, A. Thermal instability in the CGM of $\mathrm{L}_{\star}$ galaxies: Testing 'precipitation' models with the FIRE simulations. Mon. Not. R. Astron. Soc. 2021. [CrossRef]

290. Sharma, P.; McCourt, M.; Quataert, E.; Parrish, I.J. Thermal instability and the feedback regulation of hot haloes in clusters, groups and galaxies. Mon. Not. R. Astron. Soc. 2012, 420, 3174-3194. [CrossRef]

291. Nelson, D.; Sharma, P.; Pillepich, A.; Springel, V.; Pakmor, R.; Weinberger, R.; Vogelsberger, M.; Marinacci, F.; Hernquist, L. Resolving small-scale cold circumgalactic gas in TNG50. Mon. Not. R. Astron. Soc. 2020, 498, 2391-2414. [CrossRef]

292. Tumlinson, J.; Thom, C.; Werk, J.K.; Prochaska, J.X.; Tripp, T.M.; Katz, N.; Davé, R.; Oppenheimer, B.D.; Meiring, J.D.; Ford, A.B.; et al. The COS-Halos Survey: Rationale, Design, and a Census of Circumgalactic Neutral Hydrogen. Astrophys. J. 2013, 777, 59. [CrossRef]

293. Viola, M.; Cacciato, M.; Brouwer, M.; Kuijken, K.; Hoekstra, H.; Norberg, P.; Robotham, A.S.G.; van Uitert, E.; Alpaslan, M.; Baldry, I.K.; et al. Dark matter halo properties of GAMA galaxy groups from 100 square degrees of KiDS weak lensing data. Mon. Not. R. Astron. Soc. 2015, 452, 3529-3550. [CrossRef]

294. Jeltema, T.E.; Binder, B.; Mulchaey, J.S. The Hot Gas Halos of Galaxies in Groups. Astrophys. J. 2008, 679, 1162-1172. [CrossRef]

295. Bamford, S.P.; Nichol, R.C.; Baldry, I.K.; Land, K.; Lintott, C.J.; Schawinski, K.; Slosar, A.; Szalay, A.S.; Thomas, D.; Torki, M.; et al. Galaxy Zoo: The dependence of morphology and colour on environment. Mon. Not. R. Astron. Soc. 2009, 393, 1324-1352. [CrossRef]

296. Bahé, Y.M.; McCarthy, I.G.; Crain, R.A.; Theuns, T. The competition between confinement and ram pressure and its implications for galaxies in groups and clusters. Mon. Not. R. Astron. Soc. 2012, 424, 1179-1186. [CrossRef]

297. Zinger, E.; Dekel, A.; Kravtsov, A.V.; Nagai, D. Quenching of satellite galaxies at the outskirts of galaxy clusters. Mon. Not. R. Astron. Soc. 2018, 475, 3654-3681. [CrossRef]

298. Bahé, Y.M.; McCarthy, I.G. Star formation quenching in simulated group and cluster galaxies: when, how, and why? Mon. Not. R. Astron. Soc. 2015, 447, 969-992. [CrossRef]

299. Applebaum, E.; Brooks, A.M.; Christensen, C.R.; Munshi, F.; Quinn, T.R.; Shen, S.; Tremmel, M. Ultrafaint Dwarfs in a Milky Way Context: Introducing the Mint Condition DC Justice League Simulations. Astrophys. J. 2021, 906, 96. [CrossRef]

300. Blitz, L.; Rosolowsky, E. The Role of Pressure in GMC Formation II: The $\mathrm{H}_{2}$-Pressure Relation. Astrophys. J. 2006, 650, 933-944. [CrossRef]

301. Rahmati, A.; Pawlik, A.H.; Raičević, M.; Schaye, J. On the evolution of the H I column density distribution in cosmological simulations. Mon. Not. R. Astron. Soc. 2013, 430, 2427-2445. [CrossRef]

302. Gnedin, N.Y.; Draine, B.T. Line Overlap and Self-Shielding of Molecular Hydrogen in Galaxies. Astrophys. J. 2014, $795,37$. [CrossRef]

303. Marasco, A.; Crain, R.A.; Schaye, J.; Bahé, Y.M.; van der Hulst, T.; Theuns, T.; Bower, R.G. The environmental dependence of H I in galaxies in the EAGLE simulations. Mon. Not. R. Astron. Soc. 2016, 461, 2630-2649. [CrossRef]

304. Stevens, A.R.H.; Diemer, B.; Lagos, C.d.P.; Nelson, D.; Pillepich, A.; Brown, T.; Catinella, B.; Hernquist, L.; Weinberger, R.; Vogelsberger, M.; et al. Atomic hydrogen in IllustrisTNG galaxies: The impact of environment parallelled with local 21-cm surveys. Mon. Not. R. Astron. Soc. 2019, 483, 5334-5354. [CrossRef]

305. Diemer, B.; Stevens, A.R.H.; Forbes, J.C.; Marinacci, F.; Hernquist, L.; Lagos, C.d.P.; Sternberg, A.; Pillepich, A.; Nelson, D.; Popping, G.; et al. Modeling the Atomic-to-molecular Transition in Cosmological Simulations of Galaxy Formation. Astrophys. J. Suppl. 2018, 238, 33. [CrossRef]

306. Bahé, Y.M.; Crain, R.A.; Kauffmann, G.; Bower, R.G.; Schaye, J.; Furlong, M.; Lagos, C.; Schaller, M.; Trayford, J.W.; Dalla Vecchia, C.; et al. The distribution of atomic hydrogen in EAGLE galaxies: Morphologies, profiles, and H I holes. Mon. Not. R. Astron. Soc. 2016, 456, 1115-1136. [CrossRef]

307. Stevens, A.R.H.; Lagos, C.d.P.; Cortese, L.; Catinella, B.; Diemer, B.; Nelson, D.; Pillepich, A.; Hernquist, L.; Marinacci, F.; Vogelsberger, M. Molecular hydrogen in IllustrisTNG galaxies: Carefully comparing signatures of environment with local CO and SFR data. Mon. Not. R. Astron. Soc. 2021, 502, 3158-3178. [CrossRef]

308. Saintonge, A.; Catinella, B.; Tacconi, L.J.; Kauffmann, G.; Genzel, R.; Cortese, L.; Davé, R.; Fletcher, T.J.; Graciá-Carpio, J.; Kramer, C.; et al. xCOLD GASS: The Complete IRAM 30 m Legacy Survey of Molecular Gas for Galaxy Evolution Studies. Astrophys. J. Suppl. 2017, 233, 22. [CrossRef] 
309. Genel, S. How Environment Affects Galaxy Metallicity through Stripping and Formation History: Lessons from the Illustris Simulation. Astrophys. J. 2016, 822, 107. [CrossRef]

310. Bahé, Y.M.; Schaye, J.; Crain, R.A.; McCarthy, I.G.; Bower, R.G.; Theuns, T.; McGee, S.L.; Trayford, J.W. The origin of the enhanced metallicity of satellite galaxies. Mon. Not. R. Astron. Soc. 2017, 464, 508-529. [CrossRef]

311. Gupta, A.; Yuan, T.; Torrey, P.; Vogelsberger, M.; Martizzi, D.; Tran, K.V.H.; Kewley, L.J.; Marinacci, F.; Nelson, D.; Pillepich, A.; et al. Chemical pre-processing of cluster galaxies over the past 10 billion years in the IllustrisTNG simulations. Mon. Not. R. Astron. Soc. 2018, 477, L35-L39. [CrossRef]

312. Pasquali, A.; Gallazzi, A.; van den Bosch, F.C. The gas-phase metallicity of central and satellite galaxies in the Sloan Digital Sky Survey. Mon. Not. R. Astron. Soc. 2012, 425, 273-286. [CrossRef]

313. van de Voort, F.; Bahé, Y.M.; Bower, R.G.; Correa, C.A.; Crain, R.A.; Schaye, J.; Theuns, T. The environmental dependence of gas accretion on to galaxies: quenching satellites through starvation. Mon. Not. R. Astron. Soc. 2017, 466, 3460-3471. [CrossRef]

314. Watts, A.B.; Power, C.; Catinella, B.; Cortese, L.; Stevens, A.R.H. Global H I asymmetries in IllustrisTNG: A diversity of physical processes disturb the cold gas in galaxies. Mon. Not. R. Astron. Soc. 2020, 499, 5205-5219. [CrossRef]

315. Chung, A.; van Gorkom, J.H.; Kenney, J.D.P.; Vollmer, B. Virgo Galaxies with Long One-sided H I Tails. Astrophys. J. Lett. 2007, 659, L115-L119. [CrossRef]

316. Ricarte, A.; Tremmel, M.; Natarajan, P.; Quinn, T. A Link between Ram Pressure Stripping and Active Galactic Nuclei. Astrophys. J. Lett. 2020, 895, L8. [CrossRef]

317. Poggianti, B.M.; Jaffé, Y.L.; Moretti, A.; Gullieuszik, M.; Radovich, M.; Tonnesen, S.; Fritz, J.; Bettoni, D.; Vulcani, B.; Fasano, G.; et al. Ram-pressure feeding of supermassive black holes. Nature 2017, 548, 304-309. [CrossRef]

318. Troncoso-Iribarren, P.; Padilla, N.; Santander, C.; Lagos, C.D.P.; García-Lambas, D.; Rodríguez, S.; Contreras, S. The better half-Asymmetric star formation due to ram pressure in the EAGLE simulations. Mon. Not. R. Astron. Soc. 2020, 497, 4145-4161 [CrossRef]

319. Feldmann, R.; Carollo, C.M.; Mayer, L. The Hubble Sequence in Groups: The Birth of the Early-type Galaxies. Astrophys. J. 2011, 736, 88. [CrossRef]

320. Joshi, G.D.; Pillepich, A.; Nelson, D.; Marinacci, F.; Springel, V.; Rodriguez-Gomez, V.; Vogelsberger, M.; Hernquist, L. The fate of disc galaxies in IllustrisTNG clusters. Mon. Not. R. Astron. Soc. 2020, 496, 2673-2703. [CrossRef]

321. van den Bosch, F.C.; Ogiya, G. Dark matter substructure in numerical simulations: A tale of discreteness noise, runaway instabilities, and artificial disruption. Mon. Not. R. Astron. Soc. 2018, 475, 4066-4087. [CrossRef]

322. Engler, C.; Pillepich, A.; Joshi, G.D.; Nelson, D.; Pasquali, A.; Grebel, E.K.; Lisker, T.; Zinger, E.; Donnari, M.; Marinacci, F.; et al. The distinct stellar-to-halo mass relations of satellite and central galaxies: Insights from the IllustrisTNG simulations. Mon. Not. R. Astron. Soc. 2021, 500, 3957-3975. [CrossRef]

323. Pallero, D.; Gómez, F.A.; Padilla, N.D.; Bahé, Y.M.; Vega-Martínez, C.A.; Torres-Flores, S. Too dense to go through: The importance of low-mass clusters for satellite quenching. arXiv 2020, arXiv:2012.08593.

324. Jung, S.L.; Choi, H.; Wong, O.I.; Kimm, T.; Chung, A.; Yi, S.K. On the Origin of Gas-poor Galaxies in Galaxy Clusters Using Cosmological Hydrodynamic Simulations. Astrophys. J. 2018, 865, 156. [CrossRef]

325. Bahé, Y.M.; McCarthy, I.G.; Balogh, M.L.; Font, A.S. Why does the environmental influence on group and cluster galaxies extend beyond the virial radius? Mon. Not. R. Astron. Soc. 2013, 430, 3017-3031. [CrossRef]

326. Peebles, P.J.E. The Large-Scale Structure of the Universe; Princeton University Press: Princeton, NJ, USA, 1980.

327. Bond, J.R.; Efstathiou, G.; Silk, J. Massive neutrinos and the large-scale structure of the universe. Phys. Rev. Lett. 1980, 45, 1980-1984. [CrossRef]

328. Davis, M.; Efstathiou, G.; Frenk, C.S.; White, S.D.M. The evolution of large-scale structure in a universe dominated by cold dark matter. Astrophys. J. 1985, 292, 371-394. [CrossRef]

329. Kaiser, N. Clustering in real space and in redshift space. Mon. Not. R. Astron. Soc. 1987, 227, 1-21. [CrossRef]

330. Peacock, J.A.; Dodds, S.J. Reconstructing the Linear Power Spectrum of Cosmological Mass Fluctuations. Mon. Not. R. Astron. Soc. 1994, 267, 1020. [CrossRef]

331. Mead, A.J.; Heymans, C.; Lombriser, L.; Peacock, J.A.; Steele, O.I.; Winther, H.A. Accurate halo-model matter power spectra with dark energy, massive neutrinos and modified gravitational forces. Mon. Not. R. Astron. Soc. 2016, 459, 1468-1488. [CrossRef]

332. Takahashi, R.; Sato, M.; Nishimichi, T.; Taruya, A.; Oguri, M. Revising the Halofit Model for the Nonlinear Matter Power Spectrum. Astrophys. J. 2012, 761, 152. [CrossRef]

333. van Daalen, M.P.; Schaye, J.; Booth, C.M.; Dalla Vecchia, C. The effects of galaxy formation on the matter power spectrum: A challenge for precision cosmology. Mon. Not. R. Astron. Soc. 2011, 415, 3649-3665. [CrossRef]

334. Schneider, A.; Teyssier, R. A new method to quantify the effects of baryons on the matter power spectrum. J. Cosmol. Astropart. Phys. 2015, 12, 049. [CrossRef]

335. Mummery, B.O.; McCarthy, I.G.; Bird, S.; Schaye, J. The separate and combined effects of baryon physics and neutrino free streaming on large-scale structure. Mon. Not. R. Astron. Soc. 2017, 471, 227-242. [CrossRef]

336. Semboloni, E.; Hoekstra, H.; Schaye, J.; van Daalen, M.P.; McCarthy, I.G. Quantifying the effect of baryon physics on weak lensing tomography. Mon. Not. R. Astron. Soc. 2011, 417, 2020-2035. [CrossRef]

337. Debackere, S.N.B.; Schaye, J.; Hoekstra, H. How baryons can significantly bias cluster count cosmology. Mon. Not. R. Astron. Soc. 2021. [CrossRef] 
338. Mead, A.J.; Tröster, T.; Heymans, C.; Van Waerbeke, L.; McCarthy, I.G. A hydrodynamical halo model for weak-lensing cross correlations. Astron. Astrophys. 2020, 641, A130. [CrossRef]

339. van Daalen, M.P.; Schaye, J. The contributions of matter inside and outside of haloes to the matter power spectrum. Mon. Not. R. Astron. Soc. 2015, 452, 2247-2257. [CrossRef]

340. Huterer, D.; Takada, M. Calibrating the nonlinear matter power spectrum: Requirements for future weak lensing surveys. Astropart. Phys. 2005, 23, 369-376. [CrossRef]

341. Hearin, A.P.; Zentner, A.R.; Ma, Z. General requirements on matter power spectrum predictions for cosmology with weak lensing tomography. J. Cosmol. Astropart. Phys. 2012, 2012, 034. [CrossRef]

342. Puchwein, E.; Sijacki, D.; Springel, V. Simulations of AGN Feedback in Galaxy Clusters and Groups: Impact on Gas Fractions and the $\mathrm{L}_{X}$-T Scaling Relation. Astrophys. J. Lett. 2008, 687, L53. [CrossRef]

343. Chisari, N.E.; Richardson, M.L.A.; Devriendt, J.; Dubois, Y.; Schneider, A.; Le Brun, A.M.C.; Beckmann, R.S.; Peirani, S.; Slyz, A.; Pichon, C. The impact of baryons on the matter power spectrum from the Horizon-AGN cosmological hydrodynamical simulation. Mon. Not. R. Astron. Soc. 2018, 480, 3962-3977. [CrossRef]

344. van Daalen, M.P.; McCarthy, I.G.; Schaye, J. Exploring the effects of galaxy formation on matter clustering through a library of simulation power spectra. Mon. Not. R. Astron. Soc. 2020, 491, 2424-2446. [CrossRef]

345. Schneider, A.; Stoira, N.; Refregier, A.; Weiss, A.J.; Knabenhans, M.; Stadel, J.; Teyssier, R. Baryonic effects for weak lensing. Part I. Power spectrum and covariance matrix. J. Cosmol. Astropart. Phys. 2020, 2020, 019. [CrossRef]

346. Aricò, G.; Angulo, R.E.; Contreras, S.; Ondaro-Mallea, L.; Pellejero-Ibañez, M.; Zennaro, M. The BACCO Simulation Project: A baryonification emulator with Neural Networks. arXiv 2020, arXiv:2011.15018.

347. McCarthy, I.G.; Bird, S.; Schaye, J.; Harnois-Deraps, J.; Font, A.S.; van Waerbeke, L. The BAHAMAS project: The CMB-large-scale structure tension and the roles of massive neutrinos and galaxy formation. Mon. Not. R. Astron. Soc. 2018, 476, 2999-3030 [CrossRef]

348. White, S.D.M.; Navarro, J.F.; Evrard, A.E.; Frenk, C.S. The baryon content of galaxy clusters: A challenge to cosmological orthodoxy. Nature 1993, 366, 429-433. [CrossRef]

349. Pillepich, A.; Porciani, C.; Reiprich, T.H. The X-ray cluster survey with eRosita: Forecasts for cosmology, cluster physics and primordial non-Gaussianity. Mon. Not. R. Astron. Soc. 2012, 422, 44-69. [CrossRef]

350. Pierre, M.; Pacaud, F.; Adami, C.; Alis, S.; Altieri, B.; Baran, N.; Benoist, C.; Birkinshaw, M.; Bongiorno, A.; Bremer, M.N.; et al. The XXL Survey. I. Scientific motivations-XMM-Newton observing plan-Follow-up observations and simulation programme. Astron. Astrophys. 2016, 592, A1. [CrossRef]

351. Bocquet, S.; Saro, A.; Dolag, K.; Mohr, J.J. Halo mass function: Baryon impact, fitting formulae, and implications for cluster cosmology. Mon. Not. R. Astron. Soc. 2016, 456, 2361-2373. [CrossRef]

352. Pillepich, A.; Reiprich, T.H.; Porciani, C.; Borm, K.; Merloni, A. Forecasts on dark energy from the X-ray cluster survey with eROSITA: constraints from counts and clustering. Mon. Not. R. Astron. Soc. 2018, 481, 613-626. [CrossRef]

353. Wijers, N.A.; Schaye, J.; Oppenheimer, B.D. The warm-hot circumgalactic medium around EAGLE-simulation galaxies and its detection prospects with X-ray and UV line absorption. Mon. Not. R. Astron. Soc. 2020, 498, 574-598. [CrossRef]

354. Yoshida, N.; Furlanetto, S.R.; Hernquist, L. The Temperature Structure of the Warm-Hot Intergalactic Medium. Astrophys. J. Lett. 2005, 618, L91-L94. [CrossRef]

355. Biffi, V.; Dolag, K.; Böhringer, H.; Lemson, G. Observing simulated galaxy clusters with PHOX: A novel X-ray photon simulator. Mon. Not. R. Astron. Soc. 2012, 420, 3545-3556. [CrossRef]

356. Biffi, V.; Dolag, K.; Böhringer, H. Investigating the velocity structure and X-ray observable properties of simulated galaxy clusters with PHOX. Mon. Not. R. Astron. Soc. 2013, 428, 1395-1409. [CrossRef]

357. Zuhone, J.A.; Hallman, E.J. pyXSIM: Synthetic X-ray observations generator. arXiv 2016, arXiv:1608.002.

358. Dauser, T.; Falkner, S.; Lorenz, M.; Kirsch, C.; Peille, P.; Cucchetti, E.; Schmid, C.; Brand, T.; Oertel, M.; Smith, R.; et al. SIXTE: A generic X-ray instrument simulation toolkit. Astron. Astrophys. 2019, 630, A66. [CrossRef]

359. Merloni, A.; Predehl, P.; Becker, W.; Böhringer, H.; Boller, T.; Brunner, H.; Brusa, M.; Dennerl, K.; Freyberg, M.; Friedrich, P.; et al. eROSITA Science Book: Mapping the Structure of the Energetic Universe. arXiv 2012, arXiv:1209.3114.

360. Biffi, V.; Dolag, K.; Merloni, A. AGN contamination of galaxy-cluster thermal X-ray emission: Predictions for eRosita from cosmological simulations. Mon. Not. R. Astron. Soc. 2018, 481, 2213-2227. [CrossRef]

361. Oppenheimer, B.D.; Bogdán, Á.; Crain, R.A.; ZuHone, J.A.; Forman, W.R.; Schaye, J.; Wijers, N.A.; Davies, J.J.; Jones, C.; Kraft, R.P.; et al. EAGLE and Illustris-TNG Predictions for Resolved eROSITA X-Ray Observations of the Circumgalactic Medium around Normal Galaxies. Astrophys. J. Lett. 2020, 893, L24. [CrossRef]

362. Mazzotta, P.; Rasia, E.; Moscardini, L.; Tormen, G. Comparing the temperatures of galaxy clusters from hydrodynamical N-body simulations to Chandra and XMM-Newton observations. Mon. Not. R. Astron. Soc. 2004, 354, 10-24. [CrossRef]

363. Rasia, E.; Ettori, S.; Moscardini, L.; Mazzotta, P.; Borgani, S.; Dolag, K.; Tormen, G.; Cheng, L.M.; Diaferio, A. Systematics in the X-ray cluster mass estimators. Mon. Not. R. Astron. Soc. 2006, 369, 2013-2024.

[CrossRef]

364. Barnes, D.J.; Vogelsberger, M.; Pearce, F.A.; Pop, A.R.; Kannan, R.; Cao, K.; Kay, S.T.; Hernquist, L. Characterizing hydrostatic mass bias with MOCK-X. Mon. Not. R. Astron. Soc. 2021. [CrossRef] 
365. Avila, S.; Crocce, M.; Ross, A.J.; García-Bellido, J.; Percival, W.J.; Banik, N.; Camacho, H.; Kokron, N.; Chan, K.C.; AndradeOliveira, F.; et al. Dark Energy Survey Year-1 results: Galaxy mock catalogues for BAO. Mon. Not. R. Astron. Soc. 2018, 479, 94-110. [CrossRef]

366. Korytov, D.; Hearin, A.; Kovacs, E.; Larsen, P.; Rangel, E.; Hollowed, J.; Benson, A.J.; Heitmann, K.; Mao, Y.Y.; Bahmanyar, A.; et al. CosmoDC2: A Synthetic Sky Catalog for Dark Energy Science with LSST. Astrophys. J. Suppl. 2019, 245, 26. [CrossRef]

367. To, C.H.; Krause, E.; Rozo, E.; Wu, H.Y.; Gruen, D.; DeRose, J.; Rykoff, E.; Wechsler, R.H.; Becker, M.; Costanzi, M.; et al. Combination of cluster number counts and two-point correlations: validation on mock Dark Energy Survey. Mon. Not. R. Astron. Soc. 2021, 502, 4093-4111. [CrossRef]

368. Arnaud, M.; Pratt, G.W.; Piffaretti, R.; Böhringer, H.; Croston, J.H.; Pointecouteau, E. The universal galaxy cluster pressure profile from a representative sample of nearby systems (REXCESS) and the $\mathrm{Y}_{S Z}-\mathrm{M}_{500}$ relation. Astron. Astrophys. 2010, 517, A92. [CrossRef]

369. Le Brun, A.M.C.; McCarthy, I.G.; Melin, J.B. Testing Sunyaev-Zel'dovich measurements of the hot gas content of dark matter haloes using synthetic skies. Mon. Not. R. Astron. Soc. 2015, 451, 3868-3881. [CrossRef]

370. McQuinn, M. Locating the “Missing" Baryons with Extragalactic Dispersion Measure Estimates. Astrophys. J. Lett. 2014, 780, L33. [CrossRef]

371. Prochaska, J.X.; Zheng, Y. Probing Galactic haloes with fast radio bursts. Mon. Not. R. Astron. Soc. 2019, 485, 648-665. [CrossRef]

372. Gaspari, M.; Eckert, D.; Ettori, S.; Tozzi, P.; Bassini, L.; Rasia, E.; Brighenti, F.; Sun, M.; Borgani, S.; Johnson, S.D.; et al. The X-Ray Halo Scaling Relations of Supermassive Black Holes. Astrophys. J. 2019, 884, 169. [CrossRef]

373. Bogdán, Á.; Lovisari, L.; Volonteri, M.; Dubois, Y. Correlation between the Total Gravitating Mass of Groups and Clusters and the Supermassive Black Hole Mass of Brightest Galaxies. Astrophys. J. 2018, 852, 131. [CrossRef]

374. Booth, C.M.; Schaye, J. Dark matter haloes determine the masses of supermassive black holes. Mon. Not. R. Astron. Soc. 2010 405, L1-L5. [CrossRef]

375. Truong, N.; Pillepich, A.; Werner, N. Correlations between supermassive black holes and hot gas atmospheres in IllustrisTNG and X-ray observations. Mon. Not. R. Astron. Soc. 2021, 501, 2210-2230. [CrossRef]

376. Gaspari, M.; Tombesi, F.; Cappi, M. Linking macro-, meso- and microscales in multiphase AGN feeding and feedback. Nature Astronomy 2020, 4, 10-13. [CrossRef]

377. Bassini, L.; Rasia, E.; Borgani, S.; Ragone-Figueroa, C.; Biffi, V.; Dolag, K.; Gaspari, M.; Granato, G.L.; Murante, G.; Taffoni, G.; Tornatore, L. Black hole mass of central galaxies and cluster mass correlation in cosmological hydro-dynamical simulations. Astron. Astrophys. 2019, 630, A144. [CrossRef]

378. Cowie, L.L.; Binney, J. Radiative regulation of gas flow within clusters of galaxies: A model for cluster X-ray sources. Astrophys. J. 1977, 215, 723-732. [CrossRef]

379. Fabian, A.C. Cooling Flows in Clusters of Galaxies. Annu. Rev. Astron. Astrophys. 1994, 32, 277-318. [CrossRef]

380. Peterson, J.; Fabian, A. X-ray spectroscopy of cooling clusters. Phys. Rep. 2006, 427, 1-39. [CrossRef]

381. Gaspari, M.; Ruszkowski, M.; Sharma, P. Cause and Effect of Feedback: Multiphase Gas in Cluster Cores Heated by AGN Jets. Astrophys. J. 2012, 746, 94. [CrossRef]

382. Li, Y.; Bryan, G.L. Modeling Active Galactic Nucleus Feedback in Cool-core Clusters: The Balance between Heating and Cooling. Astrophys. J. 2014, 789, 54. [CrossRef]

383. Li, Y.; Bryan, G.L.; Ruszkowski, M.; Voit, G.M.; O'Shea, B.W.; Donahue, M. Cooling, AGN Feedback, and Star Formation in Simulated Cool-core Galaxy Clusters. Astrophys. J. 2015, 811, 73. [CrossRef]

384. Prasad, D.; Sharma, P.; Babul, A. Cool Core Cycles: Cold Gas and AGN Jet Feedback in Cluster Cores. Astrophys. J. 2015, 811, 108. [CrossRef]

385. Prasad, D.; Sharma, P.; Babul, A. AGN jet-driven stochastic cold accretion in cluster cores. Mon. Not. R. Astron. Soc. 2017, 471, 1531-1542. [CrossRef]

386. Prasad, D.; Sharma, P.; Babul, A. Cool-core Clusters: The Role of BCG, Star Formation, and AGN-driven Turbulence. Astrophys. J. 2018, 863, 62. [CrossRef]

387. Nemmen, R.S.; Tchekhovskoy, A. On the efficiency of jet production in radio galaxies. Mon. Not. R. Astron. Soc. 2015, 449, 316-327. [CrossRef]

388. McNamara, B.R.; Rohanizadegan, M.; Nulsen, P.E.J. Are Radio Active Galactic Nuclei Powered by Accretion or Black Hole Spin? Astrophys. J. 2011, 727, 39. [CrossRef]

389. Hummels, C.B.; Smith, B.D.; Hopkins, P.F.; O'Shea, B.W.; Silvia, D.W.; Werk, J.K.; Lehner, N.; Wise, J.H.; Collins, D.C.; Butsky, I.S The Impact of Enhanced Halo Resolution on the Simulated Circumgalactic Medium. Astrophys. J. 2019, 882, 156. [CrossRef]

390. Hopkins, P.F.; Quataert, E. How do massive black holes get their gas? Mon. Not. R. Astron. Soc. 2010, 407, 1529-1564. [CrossRef]

391. Gaspari, M.; Brighenti, F.; Temi, P.; Ettori, S. Can AGN Feedback Break the Self-similarity of Galaxies, Groups, and Clusters? Astrophys. J. Lett. 2014, 783, L10. [CrossRef]

392. Meece, G.R.; Voit, G.M.; O'Shea, B.W. Triggering and Delivery Algorithms for AGN Feedback. Astrophys. J. 2017, 841, 133. [CrossRef]

393. Vernaleo, J.C.; Reynolds, C.S. AGN Feedback and Cooling Flows: Problems with Simple Hydrodynamic Models. Astrophys. J. 2006, 645, 83-94. [CrossRef] 
394. Cielo, S.; Babul, A.; Antonuccio-Delogu, V.; Silk, J.; Volonteri, M. Feedback from reorienting AGN jets. I. Jet-ICM coupling, cavity properties and global energetics. Astron. Astrophys. 2018, 617, A58. [CrossRef]

395. Babul, A.; Sharma, P.; Reynolds, C.S. Isotropic Heating of Galaxy Cluster Cores via Rapidly Reorienting Active Galactic Nucleus Jets. Astrophys. J. 2013, 768, 11. [CrossRef]

396. Su, K.Y.; Hopkins, P.F.; Bryan, G.L.; Somerville, R.S.; Hayward, C.C.; Anglés-Alcázar, D.; Faucher-Giguère, C.A.; Wellons, S.; Stern, J.; Terrazas, B.A.; et al. Which AGN Jets Quench Star Formation in Massive Galaxies? arXiv 2021, arXiv:2102.02206.

397. Käfer, F.; Finoguenov, A.; Eckert, D.; Clerc, N.; Ramos-Ceja, M.E.; Sanders, J.S.; Ghirardini, V. Toward the low-scatter selection of X-ray clusters. Galaxy cluster detection with eROSITA through cluster outskirts. Astron. Astrophys. 2020, 634, A8. [CrossRef]

398. Pearson, R.J.; Ponman, T.J.; Norberg, P.; Robotham, A.S.G.; Babul, A.; Bower, R.G.; McCarthy, I.G.; Brough, S.; Driver, S.P.; Pimbblet, K. Galaxy And Mass Assembly: Search for a population of high-entropy galaxy groups. Mon. Not. R. Astron. Soc. 2017, 469, 3489-3504. [CrossRef]

399. Moretti, A.; Paladino, R.; Poggianti, B.M.; Serra, P.; Ramatsoku, M.; Franchetto, A.; Deb, T.; Gullieuszik, M.; Tomičić, N.; Mingozzi, M.; et al. The High Molecular Gas Content, and the Efficient Conversion of Neutral into Molecular Gas, in Jellyfish Galaxies. Astrophys. J. Lett. 2020, 897, L30. [CrossRef]

400. Tonnesen, S.; Bryan, G.L. Gas Stripping in Simulated Galaxies with a Multiphase Interstellar Medium. Astrophys. J. 2009, 694, 789-804. [CrossRef]

401. Ploeckinger, S.; Schaye, J. Radiative cooling rates, ion fractions, molecule abundances, and line emissivities including selfshielding and both local and metagalactic radiation fields. Mon. Not. R. Astron. Soc. 2020, 497, 4857-4883. [CrossRef]

402. Ahn, C.P.; Alexandroff, R.; Allende Prieto, C.; Anders, F.; Anderson, S.F.; Anderton, T.; Andrews, B.H.; Aubourg, É.; Bailey, S.; Bastien, F.A.; et al. The Tenth Data Release of the Sloan Digital Sky Survey: First Spectroscopic Data from the SDSS-III Apache Point Observatory Galactic Evolution Experiment. Astrophys. J. Suppl. 2014, 211, 17. [CrossRef]

403. Amodeo, S.; Battaglia, N.; Schaan, E.; Ferraro, S.; Moser, E.; Aiola, S.; Austermann, J.E.; Beall, J.A.; Bean, R.; Becker, D.T.; et al. Atacama Cosmology Telescope: Modeling the gas thermodynamics in BOSS CMASS galaxies from kinematic and thermal Sunyaev-Zel'dovich measurements. Phys. Rev. D 2021, 103, 063514. [CrossRef]

404. Schaan, E.; Ferraro, S.; Amodeo, S.; Battaglia, N.; Aiola, S.; Austermann, J.E.; Beall, J.A.; Bean, R.; Becker, D.T.; Bond, R.J.; et al Atacama Cosmology Telescope: Combined kinematic and thermal Sunyaev-Zel'dovich measurements from BOSS CMASS and LOWZ halos. Phys. Rev. D 2021, 103, 063513. [CrossRef]

405. Navarro, J.F.; Frenk, C.S.; White, S.D.M. A Universal Density Profile from Hierarchical Clustering. Astrophys. J. 1997, 490, 493-508. [CrossRef]

406. Lim, S.H.; Barnes, D.; Vogelsberger, M.; Mo, H.J.; Nelson, D.; Pillepich, A.; Dolag, K.; Marinacci, F. Properties of the ionized CGM and IGM: Tests for galaxy formation models from the Sunyaev-Zel'dovich effect. Mon. Not. R. Astron. Soc. 2021, 504, 5131-5143. [CrossRef]

407. Planck Collaboration; Ade, P.A.R.; Aghanim, N.; Arnaud, M.; Ashdown, M.; Atrio-Barandela, F.; Aumont, J.; Baccigalupi, C.; Balbi, A.; Banday, A.J.; et al. Planck intermediate results. XI. The gas content of dark matter halos: The Sunyaev-Zeldovich-stellar mass relation for locally brightest galaxies. Astron. Astrophys. 2013, 557, A52. [CrossRef]

408. The EAGLE team. The EAGLE simulations of galaxy formation: Public release of particle data. arXiv 2017, arXiv:1706.09899.

409. Nelson, D.; Springel, V.; Pillepich, A.; Rodriguez-Gomez, V.; Torrey, P.; Genel, S.; Vogelsberger, M.; Pakmor, R.; Marinacci, F.; Weinberger, R.; et al. The IllustrisTNG simulations: Public data release. Comput. Astrophys. Cosmol. 2019, 6, 2. [CrossRef] 